\title{
C. elegans as a model for membrane traffic
}

Ken Sato ${ }^{1 \S^{*}}$, Anne Norris ${ }^{2^{*}}$, Miyuki Sato ${ }^{1,3}$, Barth D. Grant ${ }^{2 \S}$

${ }^{1}$ Laboratory of Molecular Traffic, Institute for Molecular and Cellular Regulation, Gunma University, Maebashi, Gunma 371-8512, Japan

${ }^{2}$ Department of Molecular Biology and Biochemistry, Rutgers University, Piscataway, NJ 08854, USA

${ }^{3}$ Laboratory of Molecular Membrane Biology, Institute for Molecular and Cellular Regulation, Gunma University, Maebashi, Gunma 371-8512, Japan

\section{Table of Contents}

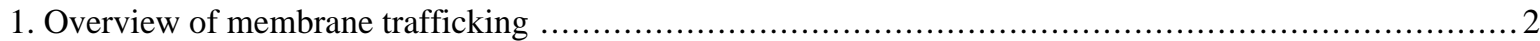

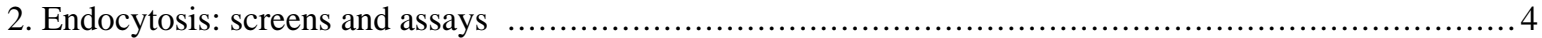

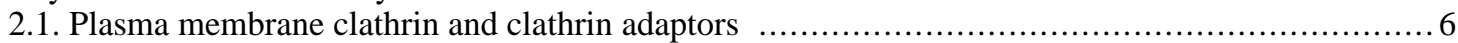

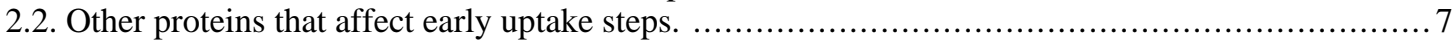

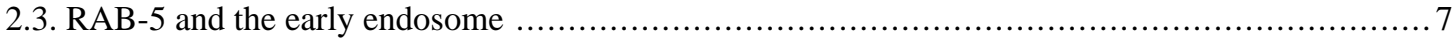

2.4. Priming downstream endosome function via early interactions at the coated pit ................... 9

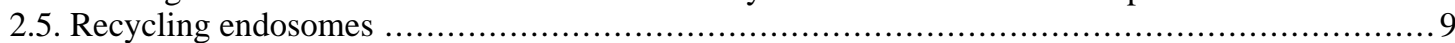

2.6. Retrograde endosome-to-Golgi transport ................................................... 14

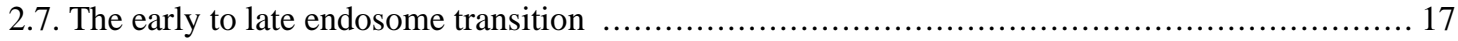

2.8. ESCRT complexes and MVB cargo sorting ................................................... 18

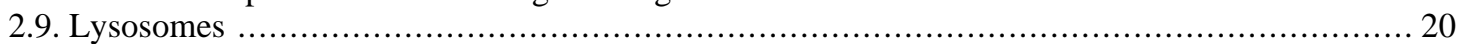

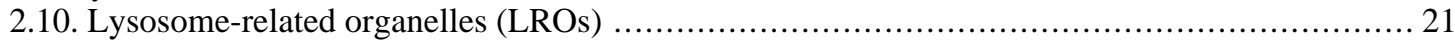

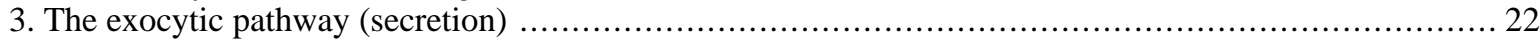

3.1. Yolk as a model cargo for studies of secretion and endocytosis . .............................. 23

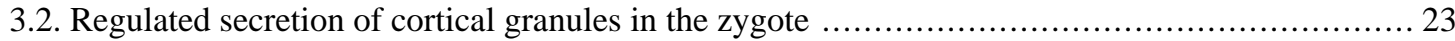

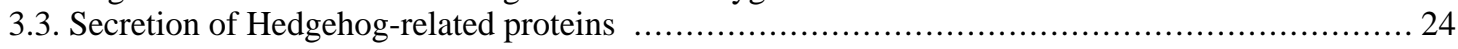

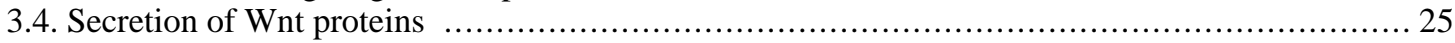

*Edited by Michel Labouesse and Donald G. Moerman Last revised October 20, 2013, Published April 25, 2014. This chapter should be cited as: Sato, K., et al. C. elegans as a model for membrane traffic (April 25, 2014), WormBook, ed. The C. elegans Research Community, WormBook, doi/10.1895/wormbook.1.77.2, http://www.wormbook.org.

Copyright: () 2014 Ken Sato, Anne Norris, Miyuki Sato, Barth D. Grant. This is an open-access article distributed under the terms of the Creative Commons Attribution License, which permits unrestricted use, distribution, and reproduction in any medium, provided the original author and source are credited.

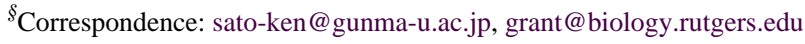

*These authors equally contributed to this work 
3.5. Function of Golgi clathrin and the AP1 clathrin adaptor complex in intestinal tubulogenesis ...... 25

3.6. Secretion of fibroblast growth factor (FGF) EGL-17 ...................................... 25

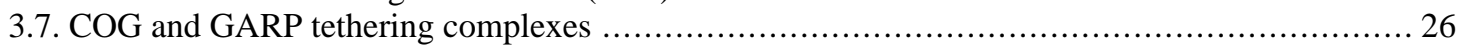

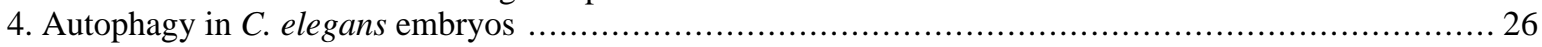

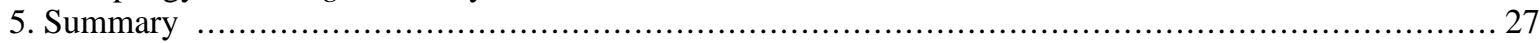

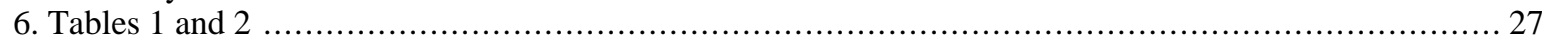

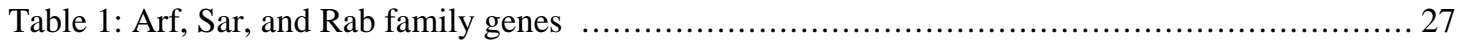

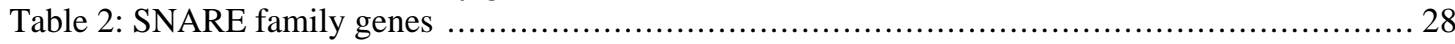

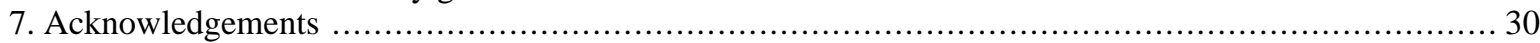

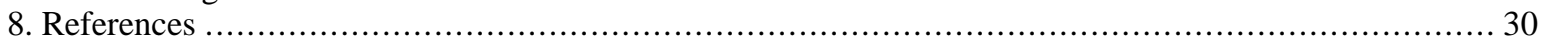

\begin{abstract}
The counterbalancing action of the endocytosis and secretory pathways maintains a dynamic equilibrium that regulates the composition of the plasma membrane, allowing it to maintain homeostasis and to change rapidly in response to alterations in the extracellular environment and/or intracellular metabolism. These pathways are intimately integrated with intercellular signaling systems and play critical roles in all cells. Studies in Caenorhabditis elegans have revealed diverse roles of membrane trafficking in physiology and development and have also provided molecular insight into the fundamental mechanisms that direct cargo sorting, vesicle budding, and membrane fisson and fusion. In this review, we summarize progress in understanding membrane trafficking mechanisms derived from work in C. elegans, focusing mainly on work done in non-neuronal cell-types, especially the germline, early embryo, coelomocytes, and intestine.
\end{abstract}

\title{
1. Overview of membrane trafficking
}

Membrane trafficking mediates transport of proteins and lipids within the endomembrane system, which is composed of small vesicles and larger intracellular organelles, including the endoplasmic reticulum (ER), the Golgi complex, endosomes, lysosomes, and autophagosomes (Figure 1) (Mukherjee et al., 1997; Delic et al., 2013). Protein transport between compartments is mediated in part by budding and fusion of small transport vesicles or tubules, fusion and fission of large organelles, and maturation processes that change organelle identity over time. Many aspects of these processes are regulated by small GTPases belonging to Arf/Sar and Rab families (Figure 2) (Chavrier and Goud, 1999). Arf/ Sar GTPases typically regulate the assembly of coat proteins on donor membranes, and are required for the formation of budding vesicles. Rab proteins most commonly regulate the later steps in vesicle transport including motor-driven vesicle movement and vesicle tethering to target membranes. The $C$. elegans genome contains 11 genes encoding Arf or Arf-like proteins, 1 gene encoding Sar1, and 31 genes encoding Rab or Rab-like proteins (Table 1, see Section 6) (Pereira-Leal and Seabra, 2001; Gallegos et al., 2012). The final step of vesicle transport, membrane fusion, is mediated by soluble N-ethylmaleimide-sensitive factor attachment protein receptors (SNAREs) (Jahn and Scheller, 2006). The SNAREs are classified functionally into v-SNAREs (also called R-SNAREs), located on the vesicles/transport intermediates, and t-SNAREs (also called Q-SNAREs), located on the target membrane. The $C$. elegans genome encodes 10 t-SNARE syntaxin homologs, 3 SNAP-25 family proteins (t-SNAREs) and 16 other SNAREs (Table 2, Section 6) (Sato et al., 2011). 


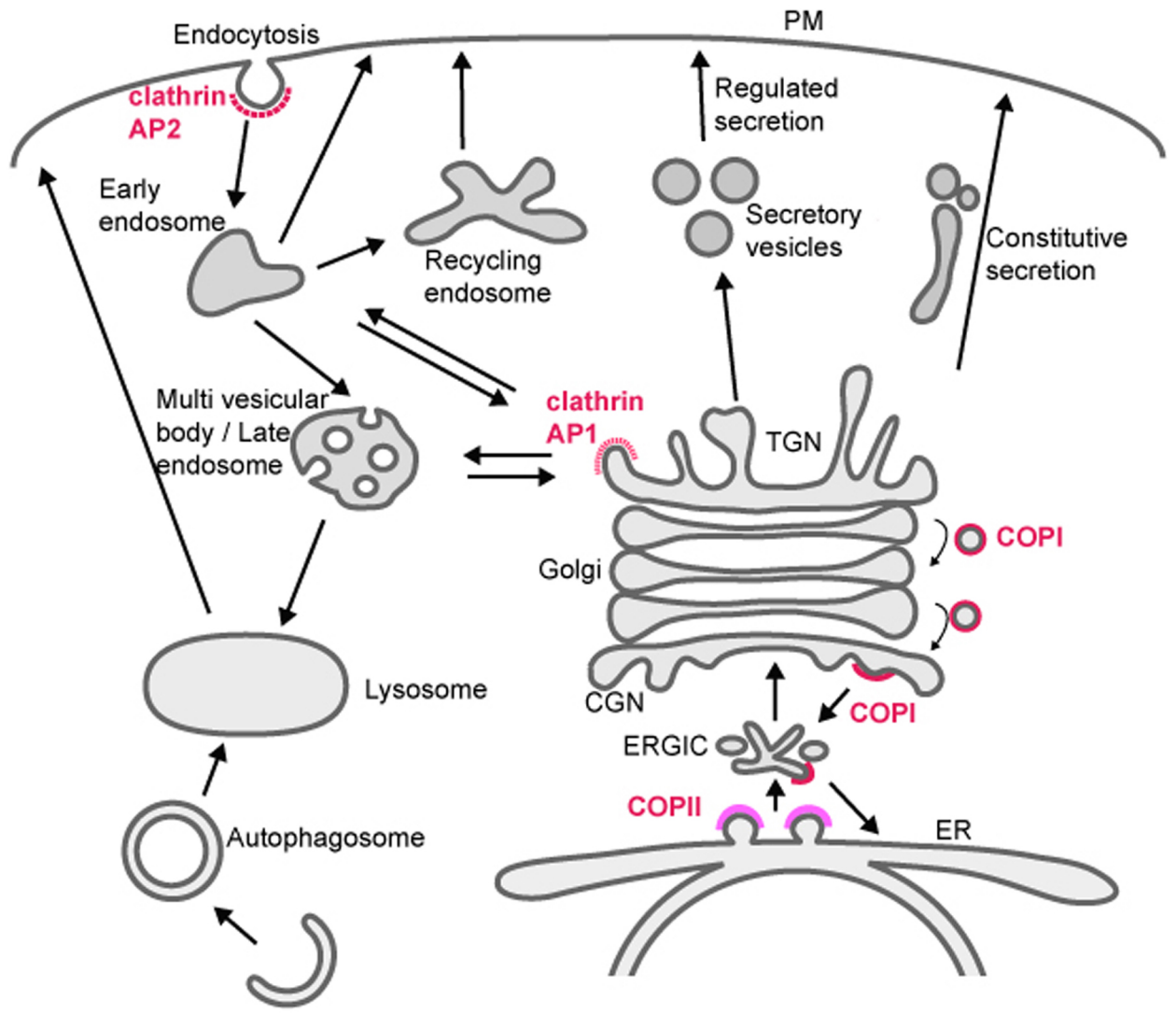

Figure 1. Membrane trafficking pathways in the endomembrane system. Transport is mediated by budding and fusion of transport carriers (vesicles or tubules), fusion of organelles, or maturation of organelles. Budding of some transport carriers is mediated by coat proteins, and representative coat proteins are indicated.

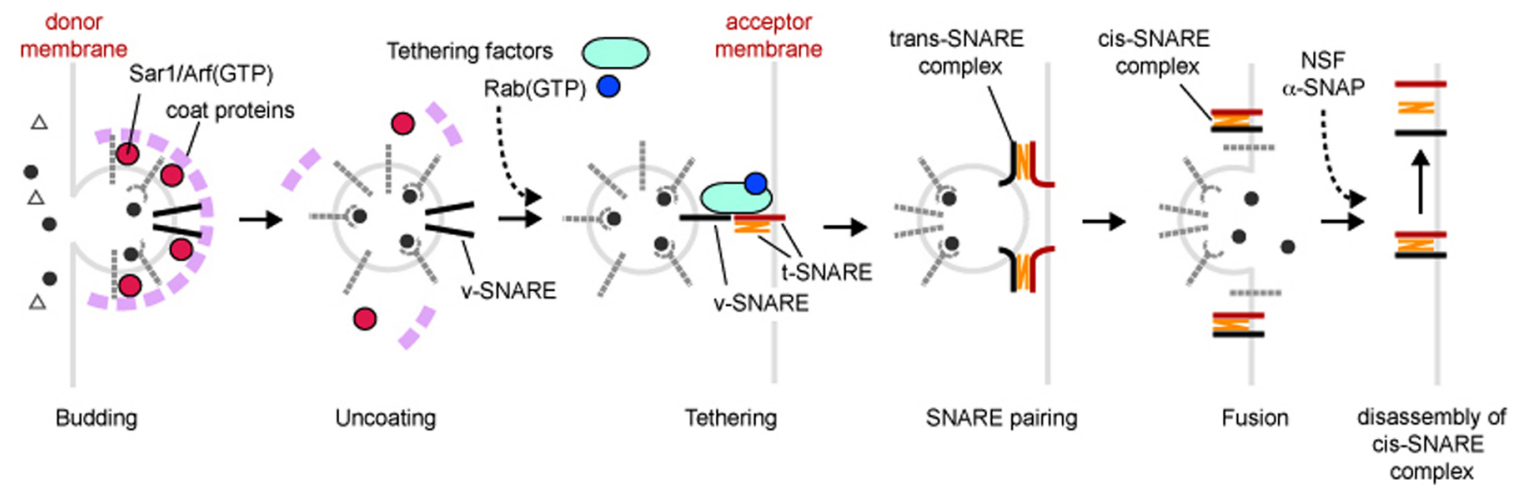

Figure 2. General mechanisms of membrane budding and fusion. Vesicles (transport carriers) are formed from the donor membrane (budding), and this process is mediated by Arf/Sar GTPases and coat proteins. Arf/Sar GTPases and coat proteins are also involved in sorting of cargo proteins into vesicles. After uncoating, Rab GTPases recruit tethering factors that help v-SNAREs on vesicles to pair with specific t-SNAREs on acceptor membranes (tethering). Then, v-SNAREs and t-SNAREs are paired (SNARE pairing), mediating the selective fusion of vesicles with the correct acceptor membrane. After fusion, SNARE complexes are disassembled by N-ethylmaleimide-sensitive fusion protein (NSF) and $\alpha$-soluble NSF attachment protein ( $\alpha$-SNAP), and are reused.

In the biosynthetic pathway transmembrane proteins and secretory proteins are synthesized in the ER. Many such proteins are then sorted into COPII coated vesicles at distinct ER-exit sites that transport cargo to the Golgi (Bonifacino and Glick, 2004). Most of these proteins are then delivered from the trans-Golgi network (TGN) to destinations such as the plasma membrane, endosomes, and lysosomes. Many proteins that function within the ER are actively recycled from the Golgi to the ER via COPI-mediated retrograde transport, a process required to maintain their ER localization. Similarly, many proteins require COPI-mediated retrograde transport from trans- to cis-Golgi compartments to maintain their usual position within the Golgi stack. Transport from the TGN to endosomes or lysosomes is mediated by clathrin-coated vesicles associated with adaptor protein complexes AP1 and 
GGA1. Most transport from the Golgi to the plasma membrane is thought to be clathrin-independent, although some secretory cargo in epithelial cells is now thought to reach the plasma membrane in an AP1/clathrin-dependent manner (Ang et al., 2004).

Cell surface membrane proteins, and extracellular macromolecules that bind to them, are internalized by endocytosis, either through cell-surface clathrin-coated pits or through a variety of poorly understood clathrin-independent mechanisms (Brodsky et al., 2001; Mayor and Pagano, 2007). Internalized cargo is transported to endosomes from which it can be sorted to lysosomes for degradation, recycled to the plasma membrane, often via a distinct recycling endosome compartment, or recycled to the TGN via retrograde recycling (endosome to Golgi transport) (Grant and Donaldson, 2009; Seaman, 2012). Very large particles, such as whole apoptotic cells, can be internalized by phagocytosis (also called engulfment). Phagosomes interact sequentially with endosomes and lysosomes to form phagolysosomes, which degrade their contents ( $\mathrm{Lu}$ and Zhou, 2012). Large cytoplasmic organelles and macromolecules can reach the lysosome for degradation via autophagy, a process by which cytoplasmic cargo is encircled by assembly of a distinct double membrane, then fusion of the autophagosome with the lysosome (Sato and Sato, 2013a; see WormBook chapter Autophagy in C. elegans).

One aspect that defines and distinguishes membrane-bound organelles from one-another is their phophatidylinositide (PI) lipid composition (Di Paolo and De Camilli, 2006). The inositol head group of PIs are often phosphorylated at defined positions around the inositol ring, giving rise to functionally distinct lipids. Many peripheral membrane proteins involved in membrane transport contain lipid binding domains with distinct preferences for particular phosphoinositide species. Such domains include the FYVE, PH, PX, and GRAM (Di Paolo and De Camilli, 2006). The distribution and importance for particular PI lipids appears largely conserved between mammals and C. elegans. Some examples include the enrichment of PI(4)P on the Golgi, PI(4,5)P2 on the plasma membrane and to a lesser extent on recycling endosomes, $\mathrm{PI}(3) \mathrm{P}$ on early endosomes, and $\mathrm{PI}(3,5) \mathrm{P} 2$ on late endosomes/lysosomes (Di Paolo and De Camilli, 2006). The control of the phosphorylation status of the inositol head group on particular membranes provides membrane identity, and has strong effects on which trafficking regulators are recruited and activated on particular organelles.

\section{Endocytosis: screens and assays}

Endocytosis is a key process that uses budding vesicles to selectively remove lipids and proteins from the plasma membrane, counterbalancing secretion, and contributing heavily to the control of plasma membrane composition (Mukherjee et al., 1997). Endocytosis also acts as a key portal by which cells take in macromolecules from the outside environment, such as nutrients and intercellular signals, typically through interaction with endocytic receptors. Endocytic uptake can be clathrin-dependent, mediated by the budding of clathrin-coated vesicles, or through a variety of poorly understood clathrin-independent vesicle budding events (Mayor and Pagano, 2007). Once internalized, primary endocytic vesicles fuse with endosomes, and extensive sorting processes within endosomes determine the final destination of cargo molecules (Maxfield and McGraw, 2004; Grant and Donaldson, 2009). In C. elegans, endocytosis has been studied most extensively in oocytes, early embryos, coelomocytes, and polarized epithelial cells of the intestine. The intestine is a polarized single layer epithelium, whereas the coelomocyte and the oocyte are generally considered to be non-polarized (see WormBook chapters Intracellular trafficking and The $C$. elegans intestine). Polarized cells, such as epithelia and neurons, maintain two completely distinct plasma membrane domains (e.g., apical versus basolateral and axon versus dendrite), which places extra demands upon the secretory and endocytic machinery (Ang and Folsch, 2012).

Several systematic genetic screens for endocytosis regulators provided important groundwork for current studies involving endocytic transport in C. elegans. One screen was the Receptor Mediated Endocytosis screen (RME screen) that assayed for defects in the uptake by oocytes of the intestinally secreted yolk protein YP170A (encoded by the vitellogenin gene vit-2, Figure 3) (Grant and Hirsh, 1999; Balklava et al., 2007). YP170::GFP adult hermaphrodites display two or three bright fluorescent oocytes, bright embryos in the uterus, and a visibly bright intestine. The pseudocoelom (body cavity) of such animals is dim. Worms defective in YP170::GFP endocytosis by oocytes display dim or dark oocytes and embryos, while the body cavity of such animals is filled with bright fluorescent YP170::GFP (Grant and Hirsh, 1999; Balklava et al., 2007). This phenotype can result from defects in the endocytosis, recycling, or cell surface delivery of the yolk receptor (RME-2) in oocytes. Analysis of yolk receptor RME-2 localization in the oocytes, using RME-2::GFP strains or anti-RME-2 antibodies, can help to differentiate between these possibilities (Balklava et al., 2007). Worms defective in secretion of YP170::GFP by the intestine display an enhanced fluorescent signal from the intestine, and dim or dark pseudocolom and oocytes/embryos. RNAi of even general secretion factors can sometimes result in accumulation of pseudocoelomic YP170::GFP, presumably because RNAi-mediated knockdown can be more efficient in the germline than in the 
intestine (Balklava et al., 2007). Traditional genetic screens using this assay identified 11 genes important for membrane traffic (Grant and Hirsh, 1999). A genome wide RNAi screen using the same assay identified hundreds of candidate endocytosis and secretion genes (Balklava et al., 2007).

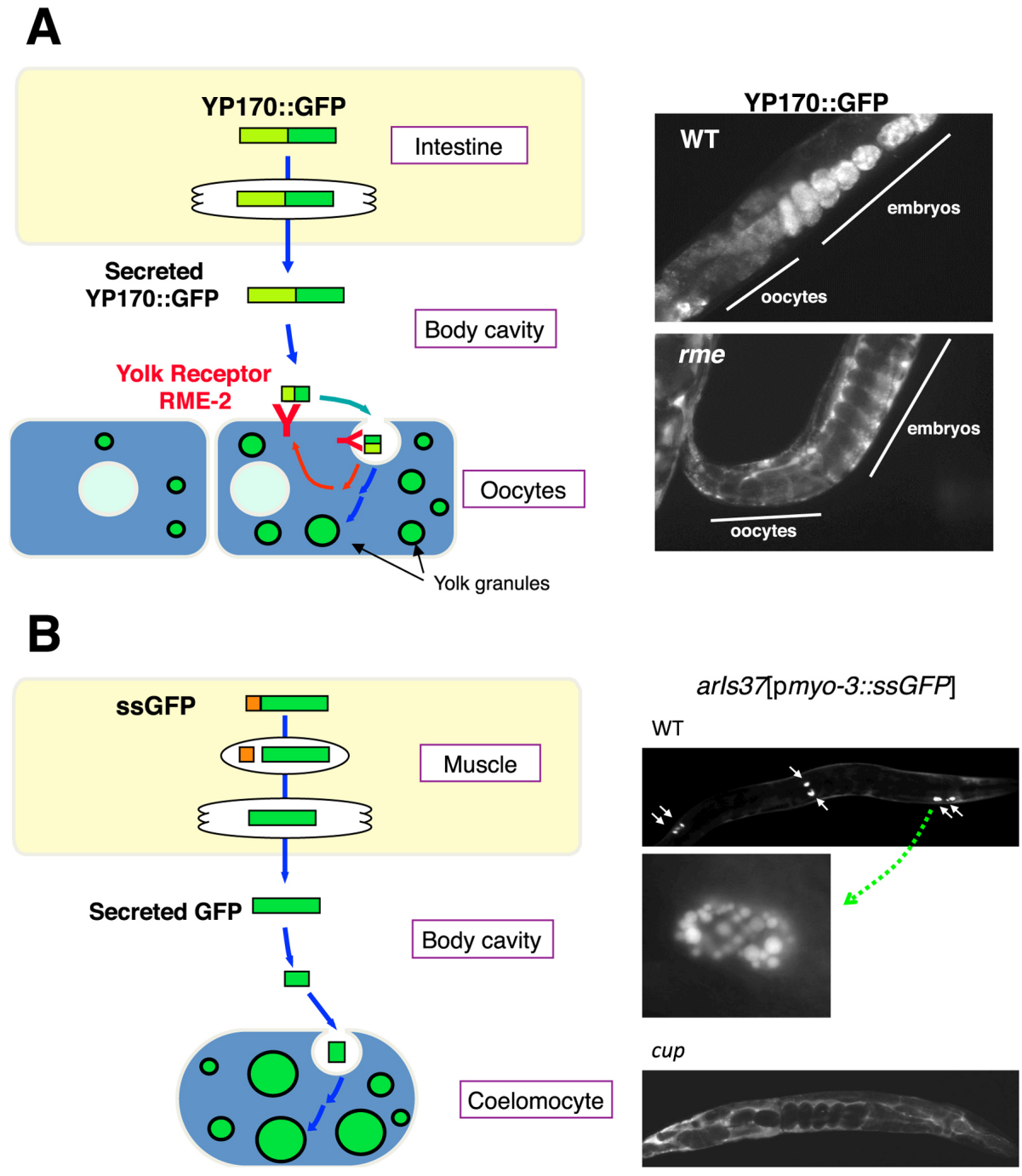

Figure 3. Endocytosis and secretion assays. (A) YP170::GFP endocytosis by oocytes. YP170::GFP is synthesized in the intestine and secreted into the body cavity from which it is endocytosed by oocytes. RME-2 is the yolk receptor expressed in oocytes. Fluorescent micrographs of wild-type and typical rme mutant worms expressing YP170::GFP are shown. (B) GFP endocytosis by coelomocytes. Signal sequence-tagged GFP is synthesized in muscle and secreted into the body cavity. Coelomocytes efficiently take up GFP from the body cavity and accumulate GFP in lysosomes. Fluorescent micrograph of wild-type and typical cup mutant worms expressing GFP are shown. High magnification image of a wild-type coelomocyte is also shown.

Another major systematic screen for endocytosis regulators was the Coelomocyte Uptake Defective (CUP) screen, which identified mutants with coelomocytes that could not endocytose green fluorescent protein (GFP) (Figure 3) (Fares and Greenwald, 2001a). In this case the reporter molecule was simple GFP with an N-terminal signal sequence, from the SEL-1 protein, expressed specifically from body-wall muscle cells. Coelomocyte cells are scavenger type cells capable of removing numerous macromolecules from the body cavity by endocytosis. Although coelomocytes in larger invertebrates such as starfish or Ascaris are motile phagocytes like mammalian macrophages, the six $C$. elegans coleomocyte cells appear to be stationary, with fixed anatomical positions, and appear to use only endocytosis, rather than phagocytosis, to clear molecules from the pseudocoelom (Fares and Greenwald, 2001a). The CUP screen identified 14 genes important for endocytic trafficking, including three genes that overlapped with the RME screen (rme-1, rme-6, and rme-8) (Fares and Greenwald, 2001a).

Other tissues in $C$. elegans have also been used to study endocytosis mechanisms, especially the polarized epithelium of the intestine, and neurons. One set of studies has focused on basolateral recycling mechanisms that return endocytosed basolateral membrane proteins from endosomes to the plasma membrane (Chen et al., 2006). 
Traditional genetic screens and targeted RNAi screens identified many proteins important for the formation or function of the autofluorescent lysosome-like organelles (gut granules) of the intestine (Hermann et al., 2005). While one recent genome-wide RNAi screen assayed for altered localization of apical intestinal membrane protein PEPT-1, apical recycling endosome protein RAB-11, and gut granules, another screened for defects in the localization of LRP-1 on hypodermal apical membranes (Winter et al., 2012; Kang et al., 2013).

\subsection{Plasma membrane clathrin and clathrin adaptors}

The best-studied route of uptake from the plasma membrane is via the clathrin-coated pit. Clathrin assembles into a lattice structure that deforms the membrane into a bud (Brodsky et al., 2001). The main structural units of the clathrin cage are the heavy chain (CHC-1) and the light chain (CLIC-1). Extensive evidence, from RNAi experiments and a temperature sensitive allele of chc- 1 , indicates that CHC-1 is essential for endocytosis and viability in C. elegans (Sato et al., 2009). CLIC-1 itself does not appear to be essential for endocytosis or viability, based upon RNAi and deletion mutant analysis (Sato et al., 2009). However, RNAi of clic-l in a chc-l temperature sensitive mutant background caused embryonic lethality at the permissive temperature, suggesting a role of CLIC-1 in at least one clathrin-mediated function in vivo (Sato et al., 2009). Data in mammalian cells indicates that clathrin light chain is more important for clathrin-mediated transport from the Golgi to endosomes, a route that delivers newly synthesized lysosomal proteins, but this has not been tested in worms (Poupon et al., 2008).

Clathrin does not bind directly to membranes or cargo. Rather, these functions are carried out by a variety of clathrin adaptors. Clathrin adaptors typically bind to short peptide motifs found in the intracellular domains of transmembrane proteins, and many clathrin adaptors also bind directly to the negatively charged plasma membrane enriched lipid PI(4,5)P2 (Traub, 2009). Some adaptors also bind to ubiquitin. Ubiquitylation of the intracellular domain of transmembrane cargo proteins can act to direct their endocytosis and sorting within the endosomal system (Traub and Lukacs, 2007). In other organisms it has been shown that clathrin adaptors can bind to ubiquitin, where mono-ubiquitin and/or K63-linked polyubiquitin are typically recognized (Lauwers et al., 2009).

There are several kinds of adaptors at the plasma membrane. One such adaptor, the heterotetrameric AP2 adaptor complex, is composed of four subunits, APA-2 ( $\alpha$-adaptin), APS-2 ( $\sigma$-adaptin), APB-1 ( $\beta$-adaptin), and APM-2/DPY-23 ( $\mu 2$-adaptin) (Traub, 2009). The APM-2 subunit is thought to bind to intracellular cargo sequences of the type YXX $\Phi$, where $\Phi$ is a large hydrophobic amino acid. One common cargo tail sequence bound by the subcomplex of $\alpha / \sigma$-adaptins is $(\mathrm{D} / \mathrm{E}) \mathrm{XXXL}(\mathrm{L} / \mathrm{I} / \mathrm{M})$, the so-called dileucine motif. Other less common motifs may also bind to AP2. Traditionally the loss of any single subunit of the AP2 complex was thought to render the whole complex unstable and non-functional. However, recent work indicates that the tetramer is composed of two heterodimers called hemicomplexes, and that each hemicomplex retains some function in the absence of the other (Doray et al., 2007; Gu et al., 2013). Biochemical experiments in mammals indicate that one hemicomplex contains $\alpha$-adaptin and $\sigma$-adaptin, while the other hemicomplex contains $\beta$-adaptin and $\mu 2$-adaptin (Doray et al., 2007). A similar arrangement is found in the related AP1 complex and is expected in the related AP3 complex.

At the genetic level in C. elegans the partial independence of the two hemicomplexes is reflected in terms of viability (Gu et al., 2013). While apa-2 and apm-2 null mutants are viable individually, apa-2; apm-2 double mutants are lethal. These results suggest that the remaining $\mu 2 / \beta$ hemicomplex retains some function when the other hemicomplex is compromised. Interestingly apa-2; apm-2 double mutant lethality can be rescued by expression of APA-2 or APM-2 in the hypodermis, indicating this tissue as the essential focus of AP2 activity. Consistent with this idea of partial hemicomplex independence, apa-2 null mutants are only mildly affected in terms of localization and abundance of APM-2 and APB-1. In contrast the APA-2 hemicomplex partner APS-2 is dramatically reduced in abundance, and the remaining APS-2 protein is mislocalized to the cytoplasm, indicating that APS-2 is unstable without APA-2. Also consistent with this model, hemicomplex partners APA-2 and APS-2 are only mildly affected in terms of localization and abundance by loss of APM-2. APB-1 is a more complex case because it is shared between the AP2 and AP1 complexes. However, even in this case, loss of the APB-1 hemicomplex partner APM-2 results in the loss of APB-1 from cellular locations that are normally occupied by AP2. Cargo experiments performed in the intestine also support the idea of partial hemicomplex independence. Known APM-2-dependent cargo MIG-14/Wntless was much less strongly affected by loss of APA-2 than loss of APM-2, and an artificial cargo bearing a dileucine internalization sequence was much more strongly affected by APA-2 than APM-2.

Another clathrin adaptor that has been studied in some depth in C. elegans is DAB-1, a PTB domain protein of the disabled protein family. PTB domains typically bind to cargo and membrane PI-lipids (DiNitto and Lambright, 2006). Disabled/Dab-family PTB domains bind to the plasma membrane-enriched lipid PI(4,5)P2, and to the typical internalization sequence found in LDL receptor family proteins, FXNPXY (Mishra et al., 2002). Consistent with a particularly important role for DAB-1 in the internalization of LDL family receptors, dab-1 mutants are strongly 
defective in the endocytosis of the yolk receptor RME-2 in oocytes, and the megalin homolog LRP-1 in the hypodermis (Kamikura and Cooper, 2003; Kamikura and Cooper, 2006; Holmes et al., 2007). DAB-1 also affects other receptor types, binding to the intracellular domain of the VAB-1 ephrin receptor, and affecting VAB-1 trafficking in the oocytes of spermless mutants (Cheng et al., 2008). DAB-1 is also important for GFP endocytosis by coelomocytes, suggesting a role in the endocytosis of scavenger receptors (Fares and Greenwald, 2001a). DAB-1 binds to other clathrin adaptors, including AP2 (Kamikura and Cooper, 2006). This interaction appears important for helping to localize DAB-1, since loss of AP2 or clathrin results in the loss of DAB-1 from the oocyte cortex (Holmes et al., 2007). This likely explains how loss of AP2 results in the reduced endocytosis of DAB-1 dependent cargo, such as RME-2, even if AP2 does not directly bind such cargo (Grant and Hirsh, 1999). The clathrin adaptor EPN-1/epsin is also important for the internalization of LRP-1 and RME-2, but is not thought to bind directly to the intracellular domain of LDL-R type receptors (Balklava et al., 2007; Holmes et al., 2007; Kang et al., 2013). DAB-1 may also play a role in intracellular clathrin mediated trafficking on the Golgi and/or endosomes. This is suggested by synthetic phenotypes with intracellular clathrin adaptors AP1 and AP2, and reports of disabled family proteins affecting receptor recycling in mammalian cells (Kamikura and Cooper, 2006; Chibalina et al., 2007; Holmes et al., 2007; Wang et al., 2008; Penheiter et al., 2010; Fu et al., 2012).

While clathrin adaptors can be relatively specific for subsets of clathrin dependent cargo, they can also be highly redundant, requiring loss of multiple adapters to noticeably affect the traffic of other cargo. An example of such redundancy comes from studies of clathrin adaptors FCHO-1/Fcho1, EHS-1/Eps15, and ITSN-1/Intersectin (FEI complex) (Mayers et al., 2013). Early studies implicated ITSN-1 in endocytosis in neuronal and non-neuronal cells, but the effects were relatively weak (Glodowski et al., 2007; Wang et al., 2008). Recent work shows that ITSN-1, FCHO-1, and EHS-1 must all be removed to observe a defect in the endocytosis of MIG-14/Wntless in the early embryo (Mayers et al., 2013). These three adaptors were shown to form a tripartite complex that appears partially redundant with the AP2 complex. For the internalization of ubiquitylated CAV-1 (caveolin-1 homolog) in the early embryo, the ITSN-1/FCHO-1/EHS-1 complex appears to be redundant with EPN-1 (Mayers et al., 2013). Loss of only epsin does not produce any noticeable effect on CAV-1. However as mentioned above, epn-1 single mutants have strong effects on LRP-1 endocytosis (Kang et al., 2013). Epsin single mutants also have strong effects on the endocytosis of Delta-family ligands in Drosophila, and studies of C. elegans GLP-1/Notch signaling in the gonad distal tip cells indicate that EPN-1/Epsin likely functions with transmembrane GLP-1 ligand LAG-2, a Delta homolog (Tian et al., 2004; Wang and Struhl, 2004).

\subsection{Other proteins that affect early uptake steps.}

As mentioned above, many clathrin adaptors bind to the lipid $\mathrm{PI}(4,5) \mathrm{P} 2$ which is important for their plasma membrane recruitment, and in some cases promotes conformational changes that encourage binding to cargo (Traub, 2009). Two proteins identified in the coelomocyte screens (CUP-4 and MCA-3/CUP-7) affect plasma membrane $\mathrm{PI}(4,5) \mathrm{P} 2$ levels, and this change in $\mathrm{PI}(4,5) 2$ may block endocytosis, at least in part, via effects on clathrin adaptors (Patton et al., 2005; Bednarek et al., 2007).

cup-4 mutant coelomocytes display a defect in a very early step of endocytosis, prior to cargo entry into early endosomes (Patton et al., 2005). The earliest defect observed in cup-4 is a reduced level of cortical PI(4, 5)P2, as revealed using a PI $(4,5) \mathrm{P} 2$ reporter, the PH domain of rat PLC- $\delta$ tagged with GFP. CUP-4 is a subunit of a non-neuronal ligand-gated ion channel that shows the highest similarity to mammalian nicotinic acetylcholine receptors (nAChRs). RNAi of another worm nAChR family protein (Y58G8A.1) also shows the Cup phenotype (Patton et al., 2005). These results suggest that CUP-4 and Y58G8A.1 assemble together to form a channel whose signaling modulates the level of $\mathrm{PI}(4,5) \mathrm{P} 2$ at the plasma membrane.

MCA-3 (CUP-7) is a plasma membrane $\mathrm{Ca}^{2+}$ ATPase (PMCA) that removes cytosolic $\mathrm{Ca}^{2+}$ to maintain proper intracellular $\mathrm{Ca}^{2+}$ levels (Bednarek et al., 2007; Brini and Carafoli, 2011). In cup-7 mutants, the level of PI(4, 5)P2 at the PM is reduced, and clathrin and RME-1 are also mislocalized. The Cup phenotype of mca-3/cup-7 can be suppressed by growing animals on plates lacking $\mathrm{Ca}^{2+}$ supplemented with calcium chelator EGTA. These results suggest that lowering extracellular calcium suppresses defects associated with the loss of MCA-3, consistent with the model that excess $\mathrm{Ca}^{2+}$ in the cytoplasm causes the endocytosis defect, perhaps through effects on $\mathrm{PI}(4,5) \mathrm{P} 2$ modifying enzymes (Bednarek et al., 2007).

\subsection{RAB-5 and the early endosome}

The small GTPase Rab5 is a master regulator of the early endocytic pathway and is considered a canonical marker for the early endosome (Bucci et al., 1992). In its active GTP-bound form Rab5 recruits a host of effector proteins to the early endosome (Woodman, 2000). Rab5 is also thought to be present at lower levels on 
clathrin-coated vesicles (Horiuchi et al., 1995). One of the key roles of Rab5 in endocytosis is to promote membrane fusion. This includes fusion of nascent endocytic vesicles with early endosomes, and the fusion of early endosomes with one another (homotypic endosome fusion) (Woodman, 2000). As with other members of the Ras superfamily, Rab5 activity is controlled by guanine-nucleotide exchange factors that activate it, and GTPase-activating proteins that inactivate it. Rab5 exchange factors are defined by the catalytic Vps9 domain (Carney et al., 2006). C. elegans RAB-5 is essential for viability and for endocytosis of YP170::GFP (Grant and Hirsh, 1999). One protein, TBC-2, has been reported as a RAB-5 GAP, while three $C$. elegans genes encode proteins containing the canonical Rab5 GEF domain Vps9 (Sato et al., 2005; Li et al., 2009; Chotard et al., 2010b). The activities of two Rab5 GEF domain Vps9 proteins RME-6 and RABX-5 are partially redundant however, since removal of either one is not lethal, and does not result in removal of all RAB-5 from membranes, whereas removal of both results in embryonic and larval lethality, and apparent loss of all RAB-5 from membranes (Sato et al., 2005). The other Vps9 domain protein in $C$. elegans, RIN-1, has no functional information currently available.

RME-6 binds to the clathrin-adaptor APA-2 and is mainly localized to clathrin-coated pits (Sato et al., 2005). Phenotypic data suggests that RME-6 functions to activate RAB-5 on the primary endocytic vesicles derived from coated-pits (Figure 4). Rab5 GEF activity associated with CCVs had been identified in vesicles purified from mammalian cells, but the particular protein responsible for this activity was first determined in C. elegans (Horiuchi et al., 1995; Sato et al., 2005). Subsequent work in mammalian cells suggests that mammalian hRME-6/Gapex5 performs a similar function (Semerdjieva et al., 2008).

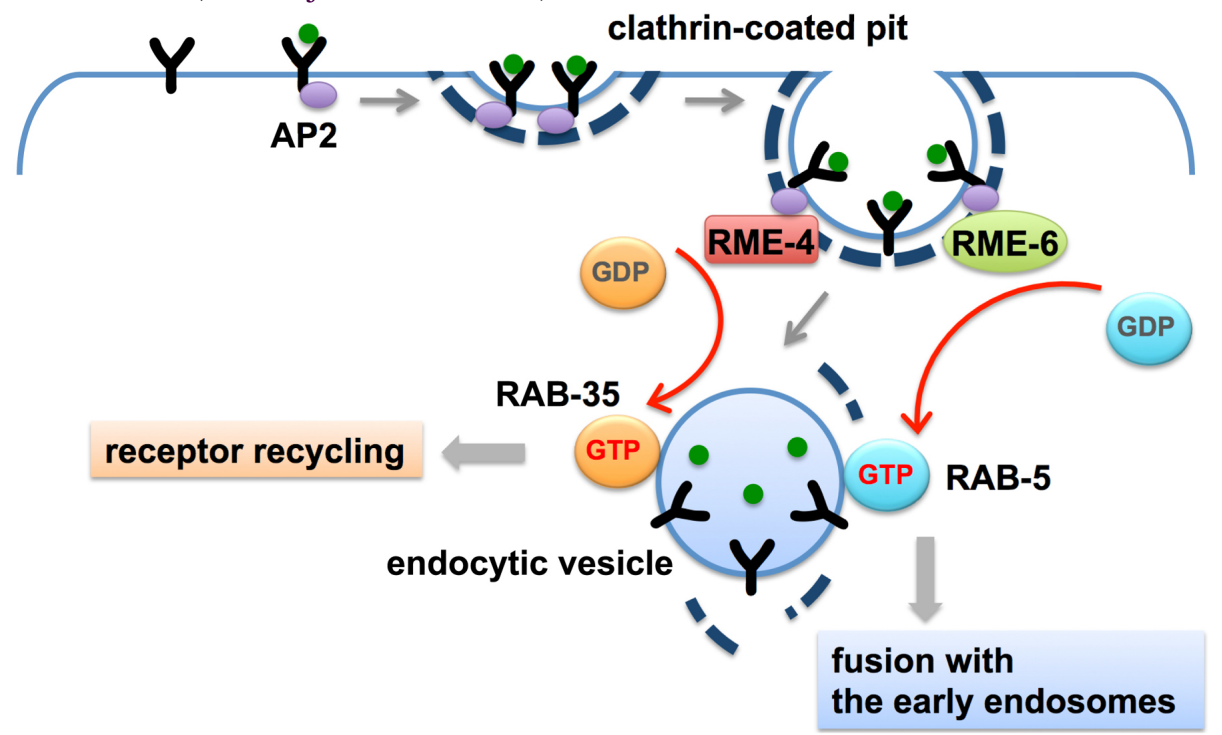

Figure 4. Priming of fusion and recycling at the clathrin-coated pit. RME-6 and RME-4 are conserved exchange factors for RAB-5 and RAB-35, respectively, and are both associated with clathrin-coated pits. RME-6-mediated activation of RAB-5 prepares the nascent vesicles to fuse with the early endosomes. RME-4-mediated activation of RAB-35 prepares for recycling of internalized receptors to the PM.

RABX-5 is thought to activate RAB-5 on early endosomes, where it is important for homotypic early endosome fusion, degradative transport toward the late endosome, and probably recycling transport as well (Woodman, 2000; Sato et al., 2005; Poteryaev et al., 2010; Dwivedi et al., 2011; Sann et al., 2012). RABX-5 is removed from early endosomes by the action of SAND-1 and its associated proteins as the degradative process proceeds, as RAB-5-positive early endosomes mature into RAB-7-positive late endosomes (Poteryaev et al., 2010). Effectors of RAB-5 that act in the fusion process include RABS-5 (Rabenosyn5) and its binding protein VPS-45, as well as VPS-34, a lipid kinase responsible for generation of PI(3)P on early endosomes (Gengyo-Ando et al., 2007). In other organisms Vps34 is known to form a complex with beclin (BEC-1 in worms) and P150 (VPS-15 in worms) (Funderburk et al., 2010). Many peripheral membrane proteins of the early endosome, including those affecting homotypic fusion, recycling, and degradation, bind to $\mathrm{PI}(3) \mathrm{P}$ and are dependent on it as a key part of their endosomal recruitment (Lindmo and Stenmark, 2006). Two lipid phosphatases, MTM-6 and MTM-9, whose substrate is PI(3)P, were isolated in a screen for defects in coelomocyte endocytosis (Dang et al., 2004). Mutations in bec- $1, \mathrm{mtm}-6$ or $\mathrm{mtm}-9$ all resulted in diffuse localization of the PI(3)P marker 2XFYVE::GFP, and/or failure of coelomocytes to endocytose GFP or labeled BSA (Dang et al., 2004; Ruck et al., 2011). The lethality of VPS-34 mutations can be suppressed by RNAi against MTM-6, consistent with the idea that VPS-34 and MTM-6 work in the same pathway, and have opposing effects on PI lipids (Xue et al., 2003). 


\subsection{Priming downstream endosome function via early interactions at the coated pit}

Once internalized, transmembrane cargo proteins enter the early endosome from which they are sorted for recycling or degradation (Grant and Donaldson, 2009). Recycling proteins return to the plasma membrane via the recycling endosome or trans-Golgi network. There is also likely to be direct recycling from early endosomes to the plasma membrane, as has been demonstrated kinetically in mammalian cells, but this route has not been confirmed in $C$. elegans. Interestingly, work in $C$. elegans indicates that some regulators of recycling and degradation are recruited early in the endocytic process by interaction with clathrin-coated pit proteins. One such protein is RME-4, a protein that binds to clathrin adaptor APA-2 and localizes to coated-pits, but appears to affect receptor recycling rather than receptor uptake (Sato et al., 2008b). RME-4 binds to the GDP-bound form of the recycling regulator RAB-35 (also known as RME-5), suggesting that RME-4 regulates RAB-35. Subsequent work on mammalian RME-4 (called Connecdenn or DENND1) showed that it is a RAB-35 exchange factor (Allaire et al., 2010; Yoshimura et al., 2010). Work in C. elegans showed that RME-4 (and clathrin) are required for recruitment of RAB-35 to early endosomes, suggesting that RME-4 recruits/activates RAB-35 on clathrin-coated vesicles, from which it is delivered to the early endosome with cargo, priming the recycling of cargo during the uptake process (Figure 4) (Sato et al., 2008b). This is reminiscent of the priming of the fusion machinery during uptake by RME-6 (Sato et al., 2005). Genetic tests in the oocyte indicated that RME-4 and RAB-35 act synergistically with recycling regulators RAB-11 and RME-1 (Sato et al., 2008b).

Recent work in the early $C$. elegans embryo indicates that multiple plasma membrane endocytic adaptors, including the FEI complex and AP2 complex, act redundantly to recruit components of the ESCRT-0 complex (HGRS-1 and STAM-1) to the cell surface (Mayers et al., 2013). This was a surprise because ESCRT-0 mainly localizes to endosomes, where it functions with several other ESCRT complexes to sort cargo into the intralumenal vesicles of the multivesicular endosome (Henne et al., 2011). This work showed that in the absence of clathrin adaptors that bind to ESCRT-0, ESCRT-0 recruitment to the plasma membrane of early embryos was strongly reduced, and the degradation of ubiquitin-modified transmembrane cargo CAV-1 was slowed (Mayers et al., 2013). Interestingly this resulted in retention of CAV-1 in early endosomes rather than the plasma membrane, suggesting that pre-assembly of ubiquitylated cargoes with the ESCRT-0 complex during uptake in coated-pits primes degradative sorting events in the endosome. Thus it appears that many downstream events in the endocytic pathway, including fusion, recycling, and degradation, are primed early, at the level of the clathrin-coated pit, enhancing the efficiency of system.

\subsection{Recycling endosomes}

Beyond the work described above in the oocyte, most research on endocytic recycling mechanisms has focused on the $C$. elegans intestine. The intestine is an interesting case for the study of membrane traffic because it is a polarized epithelial tube, and thus maintains two distinct plasma membrane domains: apical and basolateral (Figure 5) (see The C. elegans intestine). Apical junctions separate the two domains and are thought to prevent lateral diffusion of protein and lipid components between the apical and basolateral membranes. Thus strictly controlled membrane traffic is required to maintain the distinct character and functions of the two plasma membrane domains and is required to allow the movement of cargo between domains. The $C$. elegans intestine is a relatively simple epithelial tube made up of 20 epithelial cells arranged mostly in pairs to form nine rings. 


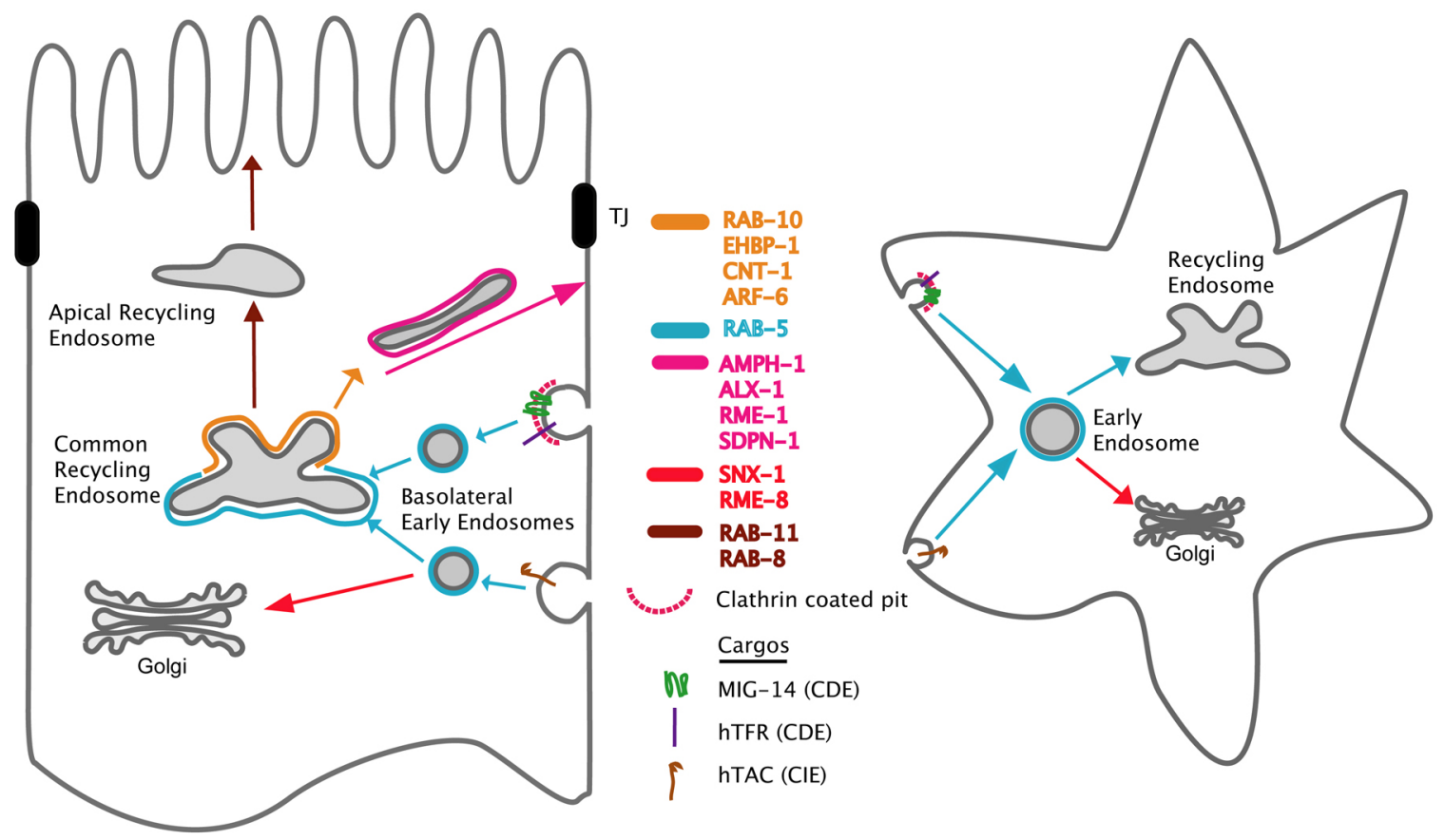

Figure 5. Cargo recycling through endocytic pathways in polarized intestinal cells and non-polarized cells. Transport pathways connecting the common recycling endosomes, apical recycling endosomes, basolateral early endosomes, the Golgi, and the PM, are indicated with different colors (left). The known regulators, which are involved in each process, are listed in the middle with each color indicating each transport pathway. Model cargos, which are endocytosed from the basolateral PM via clathrin-dependent endocytosis (CDE) or clathrin-independent endocytosis (CIE), are also indicated. In nonpolarized cells, both CDE and CIE cargos are transported to recycling endosomes or the Golgi via common early endosomes (right).

The apical membranes of the intestinal cells form the intestinal lumen, which is characterized by a microvillar brush border covered with a specialized extracellular matrix called the glycocalyx (see The C. elegans intestine). The brush border is supported from underneath by a subapical terminal web composed of actin and intermediate filaments. The basolateral membrane contacts the body cavity (pseudocoelom). Nutrients and other molecules absorbed or produced by the intestine must be exchanged via the basolateral membrane to reach the other cells of the organism. These 20 differentiated enterocyte cells are thought to be maintained for the life of the animal and are not replaced.

Several model transmembrane cargo proteins have been established to monitor basolateral traffic in the intestine (Figure 5). These include the human proteins hTAC (human IL-2 receptor $\alpha$-chain), which enters cells using clathrin-independent endocytosis (CIE), and hTfR (human transferrin receptor) that enters cells using clathrin-dependent endocytosis (CDE) (Chen et al., 2006; Shi et al., 2010; Shi et al., 2012). The endogenous $C$. elegans protein MIG-14/Wntless has also been used as a model cargo for clathrin-dependent endocytosis from the basolateral membrane of the intestine (Shi et al., 2009; Ruck et al., 2011; Gu et al., 2013). hTAC and hTfR are known to recycle using basolateral recycling endosomes. They accumulate in such endosomes in rme-1 mutant animals (Chen et al., 2006; Pant et al., 2009). MIG-14 recycles to the trans-Golgi network using a retromer-dependent mechanism and enters the degradative pathway if it fails to recycle (Pan et al., 2008; Yang et al., 2008b; Shi et al., 2009). Comparing the effects of new transport regulators on these three cargo proteins can provide preliminary insight into what steps are controlled by these regulators.

RAB-10 is a key basolateral recycling regulator in the intestine (Chen et al., 2006; Shi et al., 2010; Shi et al., 2012). rab-10 mutants display obvious vacuoles in the intestine, some large enough to observe at the dissecting-microscope level (Chen et al., 2006). These vacuoles were identified as basolateral endosomes because they accumulate fluid-phase endocytic markers from the body cavity, including GFP secreted from muscle cells and fluorescent BSA microinjected into the body cavity. Furthermore these vacuoles label for endosome markers RAB-5 and ARF-6, and accumulate high levels of the ARF-6-dependent recycling cargo hTAC::GFP (Chen et al., 2006; Shi et al., 2012). Normal-sized RAB-5-positive early endosomes also accumulate in the rab-10 mutant intestine (Chen et al., 2006). Furthermore the number of RME-1-positive basolateral recycling endosomes is greatly reduced, and those that remain lose their normal tubular morphology, now appearing punctate. RAB-10 partially colocalizes with the early endosome marker RAB-5, and often appears adjacent to RME-1-labeled tubular recycling endosomes. 
These RAB-10 positive endosomes may be the equivalent of common recycling endosomes (CREs) that are the primary location of Rab10 in polarized MDCK cells (Babbey et al., 2006). The CRE is a recycling compartment that receives and sorts cargo from basolateral and apical membranes, and is thus a specialized organelle of polarized epithelia (Wang et al., 2000). The results in C. elegans suggested that RAB-10 functions at the junction of early endosome to recycling endosome transport. rab-10 mutants do not appear to affect Golgi, late endosomes, or apical recycling endosomes, suggesting a specific role in this transport step (Chen et al., 2006). Rab10 appears to be specific for basolateral traffic in mammalian MDCK cells as well (Babbey et al., 2006).

RAB-10 function in C. elegans initially appeared limited to polarized cells. In addition to its role in the intestine, RAB-10 functions in the recycling of the postsynaptic recycling receptor GLR-1, and in the secretion of neuropeptides in neurons (polarized cells), but did not obviously affect endocytosis in non-polarized coelomocytes or oocytes (Chen et al., 2006; Glodowski et al., 2007; Sasidharan et al., 2012). This was surprising since RAB-10 is ubiquitously expressed. In fact, further analysis revealed that RAB-10 does play a role in membrane traffic in non-polarized cells, but this function is redundant with that of its closest paralog, RAB-8 (Shi et al., 2010). While $r a b-10$ and $r a b-8$ single mutants are viable and fertile, many animals depleted of both RAB-10 and RAB-8 arrest as larvae, and the surviving adults are invariably sterile. The sterility appears to result from a block in germline secretion and/or recycling, with gonads depleted of both RAB-10 and RAB-8 trapping transmembrane protein SNB-1 in intracellular vesicles. The larval arrest phenotype may also result from defects in secretion and/or endocytic recycling in other cell types when RAB-10 and RAB-8 are missing. These results suggest that RAB GTPase redundancy can differ with cell type. It remains to be determined if the redundancy in non-polarized cells represents simple biochemical interchangeability of these Rabs, or reveals redundant recycling pathways that have distinct functions in polarized cells but overlapping roles in non-polarized cells.

The relationship between RAB-10 and RAB- 8 was further revealed by analysis of a RAB-10 effector protein EHBP-1 (Shi et al., 2010). EHBP-1 contains a central calponin homology $(\mathrm{CH})$ domain, suggesting an association with actin. Actin polymerization on membranes is thought to promote membrane fission, and in some cases supports the integrity of cargo-carrying membrane tubules (Sheff et al., 2002; Kaksonen et al., 2006; Puthenveedu et al., 2010; Romer et al., 2010). EHBP-1 can bind to both RAB-10 and RAB-8 in their GTP-loaded forms, and ehbp-1 mutants strongly resemble animals lacking both RAB-10 and RAB-8 in terms of larval arrest, sterility, and intracellular trapping of SNB-1 in germ cells (Shi et al., 2010). However, in polarized cells ehbp-1 mutants strongly resemble rab-10 single mutants, with intestinal and neuronal endosome and cargo defects identical to those of rab-10. rab-8 single mutants did not produce any of these phenotypes, but rather delayed the apical transport of transmembrane protein PGP-1 in the intestine (Sato et al., 2007; Shi et al., 2010). This suggests that $C$. elegans RAB-8 plays a role in apical transport in polarized epithelia, as has been reported for Rab8 in the mouse intestine (Sato et al., 2007). Loss of EHBP-1 affects the localization of RAB-10 but not RAB-8 in the intestine and interneurons (Shi et al., 2010). Effects of EHBP-1 on the localization of RAB-10 and RAB-8 have not been tested in non-polarized cell-types.

Recent work has provided a better molecular understanding of how RAB-10 contributes to recycling endosome function. Another RAB-10-binding partner, called CNT-1, is the only C. elegans homolog of the mammalian Arf6 GTPase-activating proteins ACAP1 and ACAP2 (Shi et al., 2012). In mammals Arf6 regulates endocytic recycling and, as mentioned above, rab-10 mutants are strongly defective in the recycling of Arf6-dependent cargo protein hTAC. hTAC may report the bulk flow of membranes as they are internalized from the cell surface and recycle after passage through the endosomal system (Radhakrishna and Donaldson, 1997; Brown et al., 2001; Naslavsky et al., 2003; Naslavsky et al., 2004). Mammalian Arf6 promotes recycling in part by activating type I phophatidylinositol-4-phosphate 5 kinase, an enzyme that converts the lipid PI(4)P to PI(4,5)P2 (Radhakrishna and Donaldson, 1997; Brown et al., 2001). The C-terminal ankyrin repeats of CNT-1 bind to RAB-10(GTP), and CNT-1 colocalizes with RAB-10 and ARF-6 on intestinal endosomes in vivo (Shi et al., 2012). Furthermore, RAB-10 recruits CNT-1 to endosomal membranes: CNT-1 is diffuse in the cytoplasm in rab-10, but not $r a b-8$ or $r a b-35$, mutants. This relationship suggested that RAB-10 negatively regulates ARF-6 activity through CNT-1. Consistent with this idea, endosomal PI(4,5)P2 was elevated in cnt-1 and rab-10 mutants, and was reduced in arf-6 mutants. However, PI(4,5)P2 levels were higher in rab-10 mutants than in cnt- 1 mutants, and elevated $\mathrm{PI}(4,5) \mathrm{P} 2$ levels were only partially suppressed in $r a b-10$; arf-6 double mutants. While it is clear that RAB-10 regulates PI(4,5)P2 levels on endosomes, it must do so through ARF-6 and at least one additional mechanism. The membrane content of $\mathrm{PI}(4,5) \mathrm{P} 2$ strongly influences membrane traffic because it is a primary determinant in the recruitment of many peripheral membrane proteins including those that bend/deform membranes such as RME-1/Ehd (see below) and SDPN-1/Syndapin/Pacsin and those that nucleate membrane-associated actin polymerization such as N-WASP (Shi et al., 2012). 
Recent work also shows that $C$. elegans CED-10/Rac1 is enriched on RAB-10-positive endosomes and promotes the recycling of basolateral cargo markers hTAC::GFP and hTfR::GFP (Sun et al., 2012). The bipartite guanine nucleotide exchange factor, CED-5/Dock180- CED-12/ELMO, but not associated protein CED-2/CrkII, were also required. Interestingly, it seemed that actin-regulation was not the key CED-10-regulated process controlling recycling. Rather CED-10 was found to bind and help recruit the RAB-5 GAP TBC-2 to endosomes. Consistent with this idea, loss of TBC-2, which should elevate RAB-5 activity, or expression of constitutively active RAB-5(Q78L), also trapped recycling cargo, and overexpression of TBC-2 suppressed ced-10 mutant cargo trapping. These results indicated that an important step in the transport of cargo through the basolateral recycling endosome is the inactivation of the early endosome regulator RAB-5. This is likely to be part of a transitional process linked to the activation of the next Rab in the recycling pathway, RAB-10.

RME-1 was first discovered in the yolk transport screens, with rme-1 null mutants displaying poor endocytosis of YP170::GFP (Grant et al., 2001). Further investigation revealed that poor yolk uptake was due to poor recycling of the yolk receptor RME- 2 in oocytes. It was also noted that all rme-1 mutants displayed an unselected phenotype, progressive vacuolization of the intestine that included formation of vacuoles large enough to recognize at the level of the dissecting microscope. Subsequent work showed that these enlarged organelles labeled for ARF-6 but not RAB-5, accumulated several basolateral cargo proteins including hTAC and hTfR, and filled with basolaterally applied fluid-phase endocytosis markers but not endocytosed lipophilic dye FM 4-64 (Grant et al., 2001; Shi et al., 2012). These results indicated that RME-1 regulated a late step in basolateral recycling. RME-1 protein labeled a tubulovesicular network of endosomes just below the basolateral surface that resembled the endocytic recycling compartment (recycling endosomes) in mammalian cells (Figure 6) (Grant et al., 2001; Lin et al., 2001; Shi et al., 2012). Analysis in mammalian cells showed that the RME-1 homolog EHD1 localized to the endocytic recycling compartment and was required for recycling endosome to plasma membrane transport (Lin et al., 2001; Caplan et al., 2002). The basolateral recycling defects of rme-1 mutants, but not of rab-10 mutants, can be suppressed by loss of the PTB domain protein NUM-1/Numb. Current models indicate that NUM-1 is a negative regulator of recycling, although its mechanism is not known (Nilsson et al., 2008).
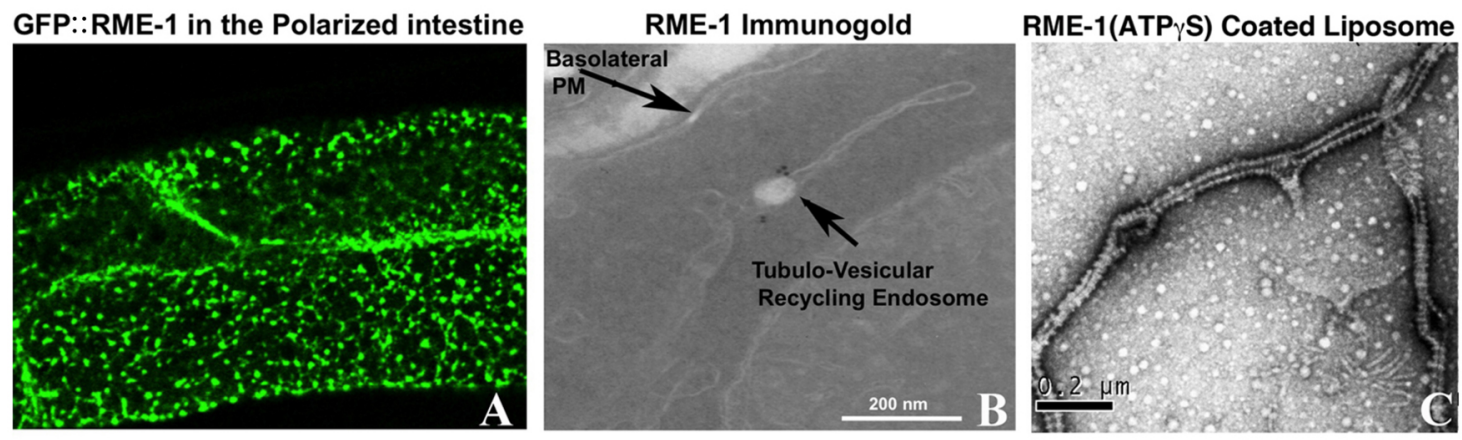

Figure 6. RME-1 is a membrane bending protein required for recycling endosome function. (A) Shows the basal surface of the intestinal cells in animal expressing GFP::RME-1. Note that the tubulovesicular network of basolateral recycling endosomes is labeled by GFP::RME-1. (B) Shows an immuno-EM image of fixed intestinal tissue using an anti-RME-1 antibody. Endogenous RME-1 is restricted to the junction of the vesicular and tubular regions of the recycling endosomes where it is likely to regulate fission of cargo-containing tubules. (C) Purified RME-1 proteins were reconstituted on synthetic liposomes. In the presence of ATP $\gamma \mathrm{S}$, RME-1 assembles in rings (striations on tubules) and squeezes the liposome membranes into narrow tubules in a manner very similar to that of Dynamin $(\mathrm{GTP} \gamma \mathrm{S})$.

A number of converging lines of research suggest that RME-1 and its mammalian homologs are membrane fission molecules that act on endosomal membranes in a manner similar to the well-studied mechanoenzyme dynamin. The large GTPase dynamin polymerizes to form spirals around the neck of clathrin-coated pits on the plasma membrane (Doherty and McMahon, 2009). Current models indicate that near simultaneous hydrolysis of GTP by the dynamin subunits within the spiral causes a dramatic change in the pitch of the spiral, constricting the neck of the pit, strongly promoting fission and release of a free vesicle (Shnyrova et al., 2013). Thus dynamin is often called a "pinchase". In addition, dynamin associates with other fission promoting molecules including BAR domain proteins that polymerize and tubulate membranes, and proteins that stimulate local actin polymerization (Doherty and McMahon, 2009). RME-1 and its mammalian homologs are ATPases, not GTPases, but the crystal structure of one of the mammalian RME-1 homologs, EHD2, showed striking structural similarities in the ATPase domain to the GTPase domain of dynamin (Lee et al., 2005; Daumke et al., 2007). This similarity is significant because the GTPase domain of dynamin is the domain that polymerizes into spirals around membranes. Consistent with this idea, it was found that purified recombinant $C$. elegans RME-1 incubated with negatively charged 
liposomes and ATP $\gamma \mathrm{S}$ in vitro produced dynamin-like spirals around the membranes (Figure 6) (Pant et al., 2009). RME-1 binding to membranes improved as the level of negative charged lipid levels increased. A combination of phosphatidylserine (PS) and PI(4,5)P2 produced the best binding of RME-1 to liposomes. RME-1 bound less well to PS and PI(4)P. Mammalian recycling endosomes are reported to be enriched in PI(4)P and PI(4,5)P2 (Jovic et al., 2009). In the $C$. elegans intestine reporters indicate that PI(4,5)P2 is enriched on RME-1-positive basolateral recycling endosomes (Shi et al., 2012).

The similarities between RME-1 and dynamin-family pinchases were extended by the discovery that RME-1 binds to C. elegans N-BAR protein amphiphysin/AMPH-1 (Pant et al., 2009). Amphiphysin was originally discovered as a binding partner of mammalian neuron-specific dynamin I (Wigge and McMahon, 1998). The interaction between RME-1 and AMPH-1 was discovered by focusing on the C-terminal, Eps15-homology (EH) of RME-1. EH-domains generally target Asn-Pro-Phe (NPF) motifs in binding partners, and the RME-1/EHD-family subfamily of EH-domains displays a preference for acidic residues after the NPF target sequence (Pant et al., 2009). C. elegans AMPH-1 has two central NPF(D/E) containing sequences, and amph-1 RNAi or deletion displaced RME-1 from recycling endosome to the cytoplasm in the $C$. elegans adult intestine. AMPH-1 colocalized with RME-1 on basolateral recycling endosomes of the intestine, and amph-1 mutants were defective in recycling cargo proteins hTfR and hTAC. The binding of RME-1 to AMPH-1 was shown to be mediated by the EH/NPF interaction, and AMPH-1 expressed from an intestine-specific promoter could rescue the hTfR recycling defect, but not if the NPF sequences were mutated to NPA. Taken together these results indicated that RME-1 and AMPH-1 function together on recycling endosomes to return recycling receptors to the basolateral cell surface.

Purified recombinant $C$. elegans AMPH-1 protein tubulated negatively charged liposomes, in a manner similar to amphiphysins from other species (Pant et al., 2009). In vitro experiments showed that RME-1 bound liposomes better when complexed with AMPH-1. Failure to help recruit RME-1 to endosomal membranes may be the reason that RME-1 appears diffusive in the intestine of amph-1 mutants. Subsequent experiments in mammalian cells showed that amphiphysin II and RME-1 homolog EHD1 colocalize on recycling endosomes, and knockdown of either amphiphysin II or EHD1 impairs transferrin recycling, indicating that RME-1 and AMPH-1 function together in mammals as they do in worms. Current models suggest that the AMPH-1 BAR domain may create membrane tubules emanating from endosomes that accumulate recycling receptors. AMPH-1 may also help to recruit RME-1 to the endosomal membrane where it assembles in rings around the neck of such tubules, pinching them off into free carriers that bring receptors to the cell surface.

Another RME-1 interacting protein is the Alix/Bro1p family protein, ALX-1 (Shi et al., 2007). ALX-1 interacts with RME-1 via two mechanisms. The EH-domain of RME-1 binds to an NPF sequence at the C-terminus of ALX-1, and the V-domain of ALX-1 binds to a YPXL sequence at the C-terminus of RME- 1 . Analysis of the $C$. elegans intestine in alx-1 mutants indicated a defect in the endocytic recycling of CIE model cargo hTAC but not CDE model cargo hTfR. This was in addition to the well-established role for Alix-family proteins in the degradative pathway (see below). In the early embryo alx-1 mutants showed strongly delayed degradation of ESCRT-dependent cargo CAV-1 (see Section 2.8). In the C. elegans adult intestine ALX-1 localized to both RME-1-positive recycling endosomes and HGRS-1-positive multivesicular endosomes. Perturbation of the NPF-containing ALX-1 binding interface for RME-1 blocked the ability of ALX-1 to rescue hTAC recycling in the intestine, but ALX-1 lacking the NPF sequence was still capable of rescuing MVE and late endosome morphology, suggesting that it remains functional for its role in the degradative pathway. Furthermore RME-1 lacking the YPXL sequence was unable to rescue hTAC recycling, indicating a requirement for RME-1 interaction with ALX-1 in the recycling pathway. RME-1 is specific for recycling: rme-1 null mutants have no apparent effect on the ESCRT-mediated degradative pathway. Thus it appears that the two functions of ALX-1 are separable and represent separate associations with RME-1 and ESCRT. Expression of truncated human Alix in HeLa cells disrupted recycling of major histocompatibility complex (MHC) class I, a known EHD1/RME-1 dependent CIE cargo, suggesting that the requirement for Alix in recycling, in addition to degradation, is conserved (Shi et al., 2007).

Another group of proteins associated with recycling endosome function are the anterior PAR proteins PAR-3, PAR-6, PKC-3 and CDC-42 (Kroschewski et al., 1999; Musch et al., 2001; Ang et al., 2003; Balklava et al., 2007). These proteins were already well known for their importance in embryonic and epithelial polarity (Goldstein and Macara, 2007). A requirement for these proteins in endocytic traffic came from the yolk transport screens in the oocyte, and further analysis showed that these proteins are also important for endocytic transport in coelomocytes (Balklava et al., 2007). CDC-42 was enriched on recycling endosomes in the coelomocyte cells and also in human HeLa cells (Balklava et al., 2007). Furthermore MHCI recycling was impaired in HeLa cells after perturbation of $\mathrm{Cdc} 42$, indicating that the requirement for $\mathrm{Cdc} 42$ in recycling is conserved. These proteins may function in regulating endosomal actin function, which is associated with membrane tubulation and fission (Balklava et al., 2007). It remains to be determined if these proteins contribute to embryonic polarity in part via endosome 
regulation, but this idea is supported by evidence that other endocytic regulators are also linked to C. elegans embryonic polarity (Nakayama et al., 2009; Hyenne et al., 2012). Furthermore, requirements for the anterior PAR complex in membrane traffic-mediated epithelial polarity have recently been shown in Drosophila, indicating a conserved role for these proteins (Georgiou et al., 2008; Leibfried et al., 2008).

In polarized epithelia like the intestine an extra recycling endosome compartment marked by RAB-11 is enriched near the apical plasma membrane (Hoekstra et al., 2004). Such apical recycling endosomes (AREs) are thought to be important for establishing and maintaining apico-basal polarity. Among the hits in a genome-wide RNAi screen for genes affecting the distribution of RAB-11 positive AREs, polarity regulators including the PAR-1 kinase and PAR-5 14-3-3 protein were identified (Winter et al., 2012). Loss of PAR-1 caused ectopic localization of RAB-11 to sites near the lateral membranes. Loss of PAR-5 produced a clustering of RAB-11-positive AREs. In addition to the normal apical RAB-11-labeleing, par-5(RNAi) induced the formation of ectopic patches of ARE localized near the basolateral plasma membrane. These patches were enriched in F-actin and apical cytoskeletal binding proteins ERM-1, EPS-8, IFB-2, and the apical junction protein AJM-1. Apical/anterior PAR proteins PKC-3, PAR-6 and PAR-3 could be found adjacent to the misplaced patches of ARE. Interestingly, the accumulation of actin and the clustering of AREs at these ectopic sites depended heavily upon the Rho-family proteins RHO-1 and CED-10, and to a lesser extent CDC-42 (Winter et al., 2012). Thus PAR-5 is important in maintaining normal intestinal polarity, with a strong effect on endosome function. The mechanism of PAR-5 action in the intestine remains to be determined.

Like phospholinositides, phosphatidylserine (PS) contributes heavily to the negative charge on cellular membranes (Sebastian et al., 2012). In fact PS is the most abundant anionic phospholipid of cell membranes and is asymmetrically arranged between the two membrane leaflets, with more PS on the cytoplasmic leaflet of cell membranes. Like PI's, PS is very important in recruiting peripheral membrane proteins involved in membrane traffic to cellular membranes. Although the charge of PS head groups is not modified by phosphorylation like PI, its abundance on the cytoplasmic leaflet can be regulated by lipid flippases that move PS between leaflets, regulating its availability for binding to cytoplasmic proteins (Sebastian et al., 2012). PS asymmetry has also been proposed to encourage membrane curvature (van Meer, 2011).

C. elegans PS is enriched on tubular membrane structures and on the cytosolic leaflet of recycling, early and late endosomes as well as lysosomes (Chen et al., 2010). The P4-ATPase TAT-1, along with its chaperone CHAT-1, maintains PS asymmetry both on the plasma membrane and on endosomal membranes (Darland-Ransom et al., 2008; Chen et al., 2010). CHAT-1 and TAT-1 associate with PS-coated tubular membranes of early endosomes, endocytic recycling compartments (ERCs) and recycling endosomes (Chen et al., 2010). Loss of TAT-1 or CHAT-1 disrupts membrane PS asymmetry and collapses the tubular membrane structure of sorting and recycling endosomes in the C. elegans intestine. Endocytic sorting of cargo is also severely affected in chat- 1 and tat- 1 mutants, causing abnormal cargo recycling and degradation, and trapping the cargo in enlarged early endosomes (Ruaud et al., 2009; Chen et al., 2010; Nilsson et al., 2011). The defects in recycling membrane traffic, and in apparent membrane charge, in tat-1 and chat-1 mutants can be suppressed by loss of DNPP-1, an aspartyl aminopeptidase (Li et al., 2013). Degradative function remains abnormal in tat-1; dnpp- 1 double mutants, and dnpp- 1 single mutants show mild defects in the degradative pathway. The DNPP-1 substrate(s) that mediate its effects remain to be identified.

\subsection{Retrograde endosome-to-Golgi transport}

At the level of the early endosome, transmembrane proteins internalized by endocytosis can be sorted to a variety of destinations, including the lysosome, recycling endosome, or Golgi (Grant and Donaldson, 2009). Traffic from endosomes to the Golgi is often referred to as retrograde transport or retrograde recycling, and is dependent upon a core protein complex called the retromer (Seaman, 2012). First identified in yeast as essential for the recycling of hydrolase receptor Vps10p from endosomes to the Golgi, the retromer (Vps35p, Vps26p, Vps29p, Vps5p, and Vps17) also functions in a similar pathway in metazoans. The equivalent proteins are required in mammalian cells for endosome to Golgi retrograde recycling of the mannose 6-phosphate receptor, another type of hydrolase receptor that carries newly produced lysosomal enzymes from the Golgi to endosomes and that must then recycle back to the Golgi. The C. elegans retromer consists of the VPS-35, VPS-26 and VPS-29 cargo recognition trimer, and the SNX-1/SNX-6 (Vps5/Vps17) obligate heterodimer (Verges, 2007). Recent work also links sorting nexin SNX-3 to this pathway in worms (Harterink et al., 2011). Another sorting nexin, Snx27 also binds to retromer and is a key protein in mammalian retromer-mediated recycling of the $\beta 2$-adrenergic receptor, glucose transporter 1 , and probably many other transmembrane proteins (Temkin et al., 2011; Steinberg et al., 2013). While the C. elegans genome encodes a clear homolog of Snx27 (SNX-27), the function of SNX-27 in worms remains to be determined. Retromer-dependent retrograde recycling has recently been linked to multiple metazoan-specific processes, such as the generation of Wnt signaling gradients, glutamate-receptor signaling and Amyloid Precursor Protein (APP) 
trafficking in the nervous system, clearance of dead apoptotic cells, and regulation of epithelial polarity (Coudreuse et al., 2006; Prasad and Clark, 2006; Korolchuk et al., 2007; Belenkaya et al., 2008; Franch-Marro et al., 2008; Pan et al., 2008; Port et al., 2008; Yang et al., 2008a; Chen et al., 2010; Lu et al., 2011; Pocha et al., 2011; Zhou et al., 2011a; Zhang et al., 2012a).

While the C. elegans genome does not encode an obvious hydrolase receptor related to yeast Vps10p or mammalian mannose 6-phosphate receptors, the retromer complex is required in C. elegans for recycling of other transmembrane proteins, including the Wnt chaperone MIG-14/Wntless and the AMPA receptor GLR-1 (Pan et al., 2008; Yang et al., 2008a; Zhang et al., 2012a). At least some components of the retromer complex are required for the clearance of apoptotic cell corpses, but there is some debate as to which components are required and whether they function to recycle the cell corpse receptor CED-1, or whether they act to promote the fusion of phagosomes with lysosomes and early endosomes by encouraging tubular contact points among these organelles (Chen et al., 2010; Lu et al., 2011).

By forming a concentration gradient, Wnt ligands provide positional information to cells in an organism, particularly anterior-posterior polarized cell divisions, cell migrations, and axon pathfinding (see WormBook chapter Wnt signaling in C. elegans). Wnt-producing cells secrete Wnt ligands using the MIG-14 transmembrane chaperone, which binds to nascent Wnt ligands such as EGL-20 in the Golgi. MIG-14 then chaperones Wnt ligands to the plasma membrane for release (Pan et al., 2008; Yang et al., 2008a). For subsequent rounds of Wnt secretion, MIG-14 must be endocytosed in a clathrin/AP2-dependent manner, and MIG-14 must be recycled to the Golgi via retrograde transport (Pan et al., 2008; Yang et al., 2008a). Blocking the endocytosis of MIG-14 blocks its function and greatly impairs Wnt secretion, resulting in Wnt phenotypes. MIG-14/Wntless endocytosis and recycling is a conserved process that has been directly demonstrated in C. elegans, Drosophila, and mammalian cells (Hausmann et al., 2007; Eaton, 2008). In the absence of retromer components VPS-35, VPS-26, or SNX-3, MIG-14 is missorted to lysosomes after endocytosis and is degraded, resulting in low cellular levels of MIG-14 and poor secretion of Wnt ligands such as EGL-20 (Pan et al., 2008; Yang et al., 2008a). This produces severe defects in Wnt signaling, including defective migration of Q-cell descendants and defective polarity of mechanosensory neurites (Coudreuse et al., 2006; Prasad and Clark, 2006; Pan et al., 2008; Yang et al., 2008a). While defects in retrograde recycling of MIG-14 result in Wnt signaling defects, any trafficking defect that inhibits MIG-14 from reaching the Golgi, or trafficking defects of Wnt receptors, can affect Wnt signaling (Pan et al., 2008; Yang et al., 2008a). Hence, only a subset of trafficking mutants that produce Wnt phenotypes affect retrograde endosome-to-Golgi traffic.

While mutations in retromer subunits vps-35 and vps-26 produce strong defects in Wnt signaling, and in the retrograde recycling of MIG-14, loss of the other core component of the retromer cargo-selective complex, VPS-29, produces only minor defects in MIG-14 sorting, and Wnt signaling is hardly affected (Coudreuse et al., 2006). However, vps-29 mutant phenotypes are strongly enhanced when combined with loss of other retrograde recycling regulators that also produce weak phenotypes on their own, such as snx-1 and $d n c-1$ (Coudreuse et al., 2006; Wassmer et al., 2009). In fact, a synthetic or enhanced Wnt-related phenotype when a mutation is combined with vps-29 is often used as supporting evidence when testing for the involvement of a new protein in retrograde transport (Coudreuse et al., 2006; Wassmer et al., 2009). Given that VPS-29 co-purifies with VPS-26 and VPS-35 in many other organisms, VPS-29 is most likely a constitutive component of the cargo recognition complex, but in $C$. elegans VPS-29 appears to play a supporting role in retrograde recycling, or may be more important for retromer cargo other than MIG-14.

Sorting nexins, defined simply by the presence of the PI(3)P lipid binding PX motif, represent a diverse array of proteins that often contain a variety of additional functional domains (Cullen and Korswagen, 2011). Sorting nexins implicated in retrograde transport include SNX-1, SNX-6 and SNX-3. A subgroup of sorting nexins contain a membrane bending/tubulating domain, the BAR domain, and are often referred to as SNX-BAR proteins (van Weering et al., 2010). C. elegans has three members of this type SNX-1, SNX-6, and LST-4, with SNX-1/SNX-6 dimers thought to participate in retromer function. SNX-BAR proteins are thought to be important for creating the characteristic retromer-coated tubules that shunt cargo from the endosome to the Golgi in mammalian cells, although tubular retromer-based carriers have not been observed in C. elegans (van Weering et al., 2010).

The SNX-1 BAR domain binds to the C-terminal third of the C. elegans DNA J-domain protein RME-8, originally identified in the yolk transport screens (Zhang et al., 2001; Shi et al., 2009). snx-1 and rme-8 mutants missort MIG-14 to lysosomes in the intestine, and display defective polarity of mechanosensory neurites, suggesting a defect in Wnt signaling (Shi et al., 2009). Interestingly, the centrally located RME-8 J-domain binds to HSP-1/Hsc70, a protein folding chaperone (Chang et al., 2004; Girard et al., 2005; Shi et al., 2009). SNX-1 and RME-8 colocalize to a subset of early endosomes that are often directly juxtaposed to Golgi ministacks (Shi et al., 
2009). This proximity may facilitate endosome to Golgi transport. J-domain proteins typically recruit and activate Hsc70 proteins, localizing their activity in the assembly and disassembly of protein complexes, such as clathrin lattices (Walsh et al., 2004; Eisenberg and Greene, 2007). Thus current models propose that RME-8 acts on subdomains of endosomes enriched in retromer complexes, disassembling clathrin lattices that encroach from neighboring endosomal subdomains that are enriched in ESCRT complexes (Shi et al., 2009). In mammalian cells the ESCRT-0 component HRS (HGRS-1) binds to a specialized flat clathrin lattice on endosomes that is thought to concentrate ESCRT complexes and degradative cargo (Raiborg et al., 2001a; Raiborg et al., 2002; Raiborg et al., 2006). Consistent with this model, mutants or RNAi of $s n x-1, r m e-8$, and $h s p-1$ mislocalize and degrade intestinally expressed MIG-14 via the lysosome, and over-accumulate endosomal clathrin (Shi et al., 2009). These results suggest that endosomes maintain distinct recycling and degradative subdomains via molecular antagonism between recycling and degradative regulatory proteins. The requirement for RME-8 in retrograde recycling, and its binding and colocalization with Snx1 and other retromer components, is conserved in mammals (Popoff et al., 2009; McGough and Cullen, 2013).

SNX-3 homologs are known to play important roles in retrograde trafficking in yeast and mammals. The yeast SNX-3 homolog Grd19 is required for the retrograde trafficking of the iron transporter complex Fet3p-Ftrlp (Strochlic et al., 2008). However, unlike vps5 (the snx-1 homolog) mutants, grd19 mutants display only minor defects in Vps10p trafficking (Voos and Stevens, 1998; Strochlic et al., 2007). Additionally, Grd19p binds directly to the Fet3p-Ftr1p cargo, leading to models stating that Grd19p/Snx3 is a cargo specific adaptor promoting the inclusion of Fet3p-Ftr1p into Vps5p/Vps17p containing retromer tubules (Strochlic et al., 2007). In mammals, Snx3 along with Vps35 affects transferrin receptor (Tfr) recycling in erythroid progenitor cells (Chen et al., 2013). Whether Snx1 is involved in this process is not known.

Recently worm SNX-3 was shown to play an important role in the trafficking of MIG-14 (Harterink et al., 2011). As in vps-35 mutants, C. elegans snx-3 mutants missort MIG-14 to the lysosome and are strongly defective in Wnt signaling (Harterink et al., 2011). The same study used mammalian tissue culture cells to perform co-immunopreciptiations with human SNX3 or SNX1, finding that while both sorting nexins co-precipitate the VPS35/29/26 trimer, SNX1 and SNX3 did not pull down each other. Additionally, in Drosophila, loss of SNX1 or SNX6 did not appear to affect Wls (MIG-14) recycling or Wnt signaling (Harterink et al., 2011 and Zhang et al., 2011). An intriguing model was suggested whereby a SNX-3/VPS-trimer complex participates in a MIG-14 recycling step that largely excludes SNX-1/SNX-6. Given the strong phenotype of the snx-3 mutant on both MIG-14 localization as well as Wnt dependent QL.d migration, a key requirement for SNX-3 in MIG-14 retrograde recycling is clear. More work will be required to determine if it acts independently of SNX-1. It will also be of great interest to determine the mechanism by which SNX-3 promotes MIG-14 recycling and to determine the full spectrum of SNX-3-dependent cargo proteins.

Genetic evidence from screens for suppressors of ipla- 1 and daf-6 suggests that SNX-1 and SNX-3 regulate the same processes (Kanamori et al., 2008; Oikonomou et al., 2012). For instance, in ipla-1 mutant worms, cortical $\beta$-catenin is delocalized in seam cells and seam-cell asymmetry is perturbed, leading to supernumerary seam cell production. RNAi knockdown of any of the retromer components vps-26, vps-29, vps-35, snx-1 or snx-3, restores normal seam cell divisions and $\beta$-catenin asymmetry (Kanamori et al., 2008).

Another suppressor screen that touches on retromer function involves sheath glial cells. These cells undergo a complex morphogenetic event, involving the formation of a specialized extracellular matrix. The sheath cells form a compartment that surrounds sensory cilia that project from the dendrites of specific amphid sensory neurons. The size of the amphid compartment is negatively regulated by DAF-6 (Oikonomou et al., 2011). daf-6 mutants display a bloated sensory compartment containing excess matrix material. Genetic screens for suppression of the overly large amphid compartment of daf-6 mutants identified snx-1, snx-3 and vps-29 (Oikonomou et al., 2012). Surprisingly snx-6, vps-35 and vps-26 mutants did not suppress daf-6 (Oikonomou et al., 2012). It will be of great interest to determine how retromer components play a role in amphid sensory compartment morphogenesis and to determine if SNX-1, SNX-3, and VPS-29 act together in a distinct pathway independent of retrograde recycling.

Until quite recently, almost nothing was known about the role of the retromer complex in neurons. The AMPA-type glutamate receptor (AMPAR) GLR-1, a channel protein required for excitatory postsynaptic response in C. elegans interneurons, was recently found to require retrograde recycling (Zhang et al., 2012a). In C. elegans, any reduction in AMPAR synaptic abundance, due to mutations in the gene itself or defects in GLR-1 protein trafficking, leads to reduced nose-touch mechanosensitivity and a depressed frequency of spontaneous reversals (Burbea et al., 2002; Shim et al., 2004; Glodowski et al., 2005). In the absence of retromer components VPS-35, SNX-1, RME-8, or the small GTPase RAB-6.2, nose-touch response is decreased (Zhang et al., 2012a). In animals lacking these retrograde trafficking regulators, GLR-1 levels are strongly reduced and the remaining GLR-1 protein is mislocalized (Zhang et al., 2012a). GLR-1 levels but not GLR-1 function can be restored by simultaneously 
blocking the ESCRT-mediated degradative pathway via expression of a dominant negative VPS-4 protein. Loss of RAB-6.2 effector LIN-10, a protein that also binds directly to GLR-1, causes endosomal accumulation of GLR-1. While RAB-6.2 colocalizes extensively with AMAN-2, a resident Golgi protein, it localizes adjacent to RME-8 and SNX-1 labeled endosomes in the GLR-1 expressing interneurons. LIN-10 colocalizes extensively with RME-8, and is juxtaposed to both RAB-6.2 and Golgi markers. LIN-10 has been proposed to function in both the recycling and degradation of GLR-1 (Zhang et al., 2012a).

The lipid PI(3)P plays a role in all trafficking pathways that pass through the early endosome, and PI(3)P is clearly important for retrograde recycling (Lindmo and Stenmark, 2006). Many important endosome regulators are reported to bind to PI(3)P including sorting nexins, HRS, and RME-8 (Raiborg et al., 2001b; Yu and Lemmon, 2001; Xhabija et al., 2011). Increases in the levels of PI(3)P via mutations in PI(3)P phosphatases $m t m-6$ or $m t m-9$ both lead to decreased Wnt signaling and decreases in MIG-14 levels (Silhankova et al., 2010). RNAi against the components of the VPS-34 lipid PI-3 kinase complex (BEC-1, VPS-15 and VPS-34) can suppress the Wnt signaling defects of $m t m-6$, but also have phenotypes of their own. Mutations in bec-1, which decreases the levels of PI(3)P, lead to an intracellular accumulation of MIG-14 as well as mislocalization of MIG-14 recycling determinants RME-8 and SNX-1 (Ruck et al., 2011). Hence, the right balance of PI(3)P is crucial.

Recent work also suggests that retrograde transport can be used to degrade misfolded membrane proteins. CUP-2 is one of two worm Derlin homologs (Schaheen et al., 2009). Derlin proteins localize mainly to the ER membrane and function in ER-associated degradation (ERAD) in mammalian and yeast systems. In cup-2 mutants, the unfolded protein response (UPR) is activated, suggesting accumulation of ERAD substrates in the ER (Schaheen et al., 2009). In addition to this ER-associated phenotype, cup-2 mutants abnormally accumulate CUP-7/MCA-3 on the plasma membrane. Similar accumulation of transmembrane proteins on the plasma membrane was observed when Derlin-1 was knocked down in RAW264.7 macrophage cells. Furthermore, when misfolding of surface transmembrane proteins was artificially induced in the RAW264.7 cells, misfolded surface membrane proteins were degraded in a Derlin-1- and proteasome-dependent manner (Schaheen et al., 2009). CUP-2 and Derlin-1 seem to localize to endosomes in addition to the ER. These observations imply that CUP-2 and Derlin- 1 are involved in the quality control of transmembrane proteins at the plasma membrane, or in endocytic compartments (Schaheen et al., 2009). It was further reported that CUP-2 physically interacts with SNX-1, a subunit of the retromer complex, potentially explaining how CUP-2 is retrieved from endosomes to the Golgi (Dang et al., 2011). It was proposed that CUP-2/Derlin would further transport misfolded cargo to the ER and retranslocate it to the cytoplasm for proteasome-dependent degradation (Schaheen et al., 2009; Dang et al., 2011).

\subsection{The early to late endosome transition}

Transport of cargo from the early endosome to late endosome (EE to LE) is not mediated by small vesicle transport. Rather, as some material leaves the early endosome for recycling, the remainder of the early endosome matures into a late endosome (Rink et al., 2005). An important part of this maturation process involves a switch in the dominant Rab GTPase on the endosome. During early to late endosome maturation, the master early endosome regulator RAB-5 is replaced by the master late endosome regulator RAB-7 (Rink et al., 2005). It is during this transition that ubiquitylated transmembrane cargo is segregated into endosomal microdomains containing the ESCRT (endosomal sorting complex required for transport) proteins that act to create vesicles that bud away from the cytoplasm, producing intralumenal vesicles (ILVs) (Hurley, 2010). The ubiquitylated cargo is sorted into these ILVs, creating the multivesicular endosome (also called the multivesicular body or MVB). The late endosome eventually fuses with pre-existing lysosomes, and the internal vesicles are consumed (Katzmann et al., 2002). In some cases multivesicular endosomes can fuse with the plasma membrane, and the small ILVs are released to the outside environment. Vesicles released in this manner are called exosomes (Bobrie et al., 2011). Work on C. elegans RAB-2/UNC-108 indicates that it localizes to early and late endosomes, and that loss of RAB-2 delays the exit of cargo from early endosomes, delaying cargo degradation in the lysosome of embryos and coelomocytes (Chun et al., 2008; Guo et al., 2010). The mechanism by which RAB-2 affects degradative trafficking awaits further study.

While the Rab5 to Rab7 transition was originally identified in mammalian cells, work in C. elegans was important in identifying key mechanisms that drive this process (Figure 7) (Kinchen and Ravichandran, 2010; Poteryaev et al., 2010). Key transitions of cargo along membrane trafficking pathways are often accomplished as part of a Rab GTPase cascade (Hutagalung and Novick, 2011). In its simplest form, Rab cascades involve an early acting Rab that promotes recruitment and activation of a later acting Rab. In the well-studied examples in yeast, the early acting Rab recruits an exchange factor for the later acting Rab (Rivera-Molina and Novick, 2009; Hutagalung and Novick, 2011). The later acting Rab then promotes the removal of the earlier acting Rab, typically by recruiting a GAP for the early acting Rab. Thus over time the early acting Rab is replaced by the later acting Rab, and the direction of transport becomes irreversible. 


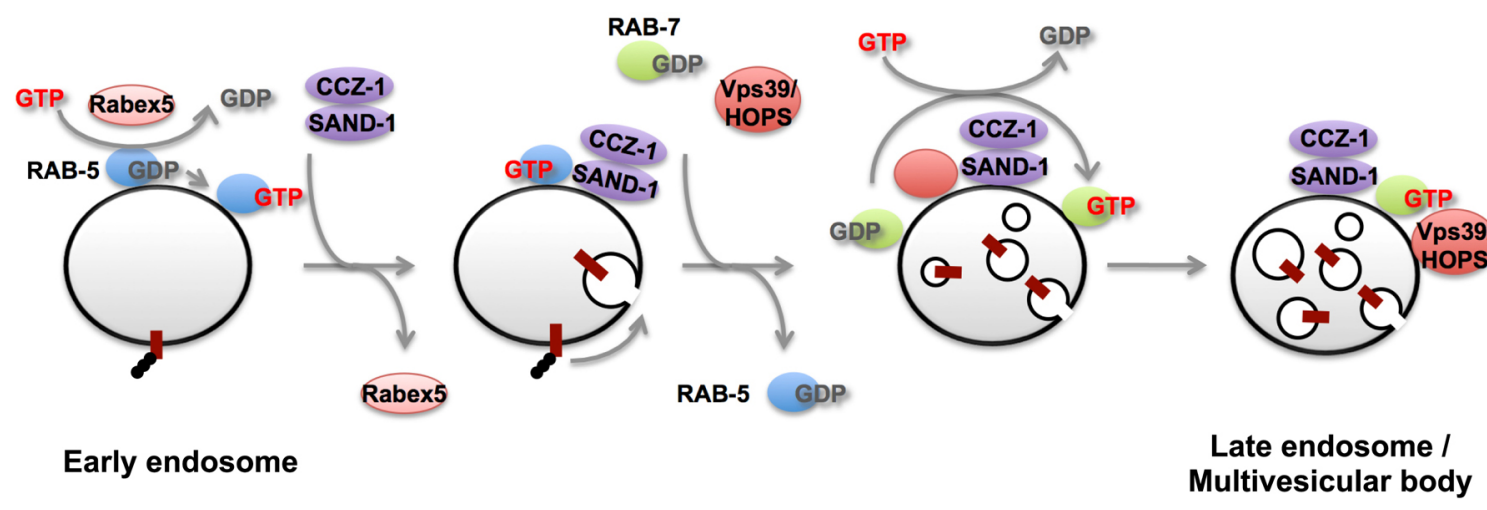

Figure 7. SAND-1 promotes RAB-5 to RAB-7 conversion during endosome maturation. RAB-5 is activated by Rabex-5 (RABX-5) on endosomes; activated RAB-5 recruits its effectors including PI 3-kinase Vps34. SAND-1/CCZ-1 is then recruited by binding GTP-bound RAB-5 and PI(3)P on the early endosomes. SAND-1 displaces of Rabex-5 from the membrane and enhances dissociation of RAB-5 from the early endosomes. SAND-1/CCZ-1 also binds to HOPS subunits and is likely to activate RAB-7 directly, thereby promoting assemble of RAB-7 and its effector HOPS on endosomes.

C. elegans SAND-1/Mon1 plays a critical role in the early to late endosome transition. sand-1 mutants are defective in degrading endocytic cargo such as yolk in embryos or GFP endocytosed by coelomocytes (Poteryaev et al., 2007). Endosomes in sand-1 mutant coelomocytes are enlarged and label with early and late endosome markers (Poteryaev et al., 2007). Further analysis showed that SAND-1 plays a dual role in the endosome maturation process (Poteryaev et al., 2010). SAND-1 acts to remove RABX-5 from the endosome, presumably blocking new recruitment/activation of RAB-5 on the endosome. Meanwhile SAND-1 acts to recruit the later acting GTPase RAB-7, a protein that directly promotes acquisition of late endosomal properties (Poteryaev et al., 2010). Recent data on the yeast SAND-1 homolog Mon1 suggests that it is a Ypt7p (RAB-7) exchange factor, directly activating the late endosomal Rab (Nordmann et al., 2010). In addition, SAND-1 appears to bind directly to the early endosomal lipid PI(3)P, so recruitment of SAND-1 is likely encouraged by the activity an early acting RAB-5 effector, VPS-34, a lipid kinase that produces PI(3)P (Poteryaev et al., 2010).

A similar process involving SAND-1 and its binding partner CCZ-1 occurs during phagosome maturation, after engulfment of apoptotic cell corpses (Kinchen and Ravichandran, 2010). In sand-1 or $c c z-1$ mutants engulfed apoptotic cells fail to be degraded. Apoptotic cell-containing phagosomes in sand-1 mutants appear to recruit RAB-5 normally, but fail to lose RAB-5 and gain RAB-7 as would normally occur. Work with mammalian SAND-1 homolog Mon1 showed that it binds to GTP-bound Rab5, and showed that a Mon1-Ccz1 complex binds to Rab7 and promotes Rab7 activation. Thus phagosome maturation shares regulators and mechanisms with early to late endosome maturation.

In addition to its connection to recycling processes, the Rab-GAP protein TBC-2, has also been implicated in the early to late endosome maturation process ( $\mathrm{Li}$ et al., 2009; Chotard et al., 2010a; Chotard et al., 2010b; Sasidharan et al., 2012; Sun et al., 2012). In vitro TBC-2 has the highest GAP activity toward RAB-5 but can also affect RAB-7 (Chotard et al., 2010a). tbc-2 mutants produce enlarged LMP-1-positive late endosomes that resemble those formed upon expression of constitutively active (GTPase defective) RAB-5(Q78L) (Chotard et al., 2010a). The $t b c$-2 phenotype is thought to occur because overly abundant GTP-bound RAB-5 hyperactivates RAB-7 through the RAB-5 to RAB-7 cascade involving SAND-1. Consistent with this idea, the formation of the enlarged LMP-1-labeled late endosomes is suppressed by RNAi of RAB-7 or components of the RAB-7 effector complex HOPS (Chotard et al., 2010a). Interestingly, $t b c-2$ mutants are also defective in phagosome maturation, failing to lose RAB-5 and gain RAB-7 (Li et al., 2009). This work was the first to indicate that TBC-2 is a RAB-5 GAP, and highlights similarities and differences between endosomes and phagosomes, since loss of TBC-2 appears to have opposing effects on RAB-7 in the two pathways.

\subsection{ESCRT complexes and MVB cargo sorting}

The formation of ILVs, and the sorting of membrane proteins into them on late endosomes, is regulated by the ESCRT machinery, consisting of four hetero-oligomeric protein complexes (ESCRT-0, -I, -II, -III) (Williams and Urbe, 2007; Hurley, 2010). In the case of yeast, ESCRT-0 first binds both to ubiquitylated membrane proteins and the cytoplasmic leaflet of endosomal membranes. The other ESCRT complexes are then recruited sequentially onto the endosomal membrane, where they act to produce membrane buds protruding from the limiting endosomal membrane toward the inside of the endosome, and sort ubiquitylated membrane proteins into the buds that will become ILVs. Finally, the complex of Vps4p, the AAA ATPase, with Vta1p dissociates ESCRT III from the endosomal membrane. The $C$. elegans genome contains ESCRT-0 (HGRS-1/VPS-27, STAM-1/PQN-19), ESCRT-I 
(VPS-23/TSG-101, VPS-37, MVB-12, VPS-28), ESCRT-II (VPS-36, VPS-22, VPS-25), ESCRT-III (VPS-20, VPS-32, VPS-24, VPS-2), and VPS-4 (Michelet et al,, 2010). Among these components, knockdown of hgrs-1/vps-27, vps-23, vps-36, vps-32 and vps-4 results in lethality at variable developmental stages (Roudier et al., 2005; Michelet et al., 2009), indicating that the ESCRT machinery is essential for development. ESCRT-0 recognizes ubiquitylated cargos and induces cargo clustering on the membrane (Wollert and Hurley, 2010). $C$. elegans ESCRT-0 consists of HGRS-1 and STAM-1, both of which mainly localize to endosomes (Mayers et al., 2011). HGRS-1 contains a double ubiquitin interacting motif (DUIM) while STAM-1 contains a Vps27/Hrs/STAM (VHS) domain and an ubiquitin binding domain (UBD). Although both proteins bind to ubiquitin simultaneously, the DUIM of HGRS-1 shows a 2-fold higher affinity for ubiquitin than the UBDs of STAM-1, suggesting that HGRS-1 is the major ubiquitin-binding protein in ESCRT-0. Deletion of hgrs-1 is lethal but stam-1 knockout animals are viable, albeit with poor fertility and a high level of embryonic lethality, suggesting that STAM-1 functions as an accessory protein. When recombinant HGRS-1 and STAM-1 were incubated with unilamellar liposomes in vitro, they formed both heterodimers and heterotetramers in binding to the membrane (Mayers et al., 2011). In addition, ESCRT-0 also exists as a 2:2 heterotetramer of HGRS-1 and STAM-1 in vivo. These suggest that ESCRT-0 forms a heterotetramer when bound to membranes and facilitates clustering of ubiquitylated cargos within microdomains of the endosomal membrane by binding to its cargos through multiple UBDs simultaneously.

HGRS-1/VPS-27 has been studied in vivo in more detail (Roudier et al., 2005). HGRS-1/VPS-27 localizes mainly to endosomes, and its depletion results in accumulation of enlarged endosomal structures in various tissues including the hypodermis and intestine. Depletion of HGRS-1/VPS-27 also results in an increase in markers of autophagy. In addition, hgrs-1/vps-27 mutant larval arrest is often associated with a defect in molting.

ESCRT-I, -II, and -III are thought to be required not only for sorting and concentration of cargos into ILVs, but also for the formation of the ILVs. C. elegans ESCRT-II binds to VPS-20 of ESCRT-III, and this complex binds specifically to highly curved membranes on synthetic liposomes in vitro, suggesting that it senses the curvature of lipid bilayer (Fyfe et al., 2011). C. elegans VPS-32 of ESCRT-III also binds to liposomes and distributes as individual particles throughout the lipid bilayer. When VPS-32 is incubated with ESCRT-II and VPS-20, it forms filamentous structures, which specifically bind to the edges of highly curved membranes. In the absence of protein, some gaps occured on the flat supported lipid bilayer and filled with membrane over time. In the presence of ESCRT proteins, such gaps on the supported lipid bilayer are closed more rapidly and uniformly, suggesting that ESCRT-III promotes remodeling of the lipid bilayer (Fyfe et al., 2011). Recent studies have shown that ESCRT-I and ESCRT-II, in combination, deform the membrane into buds. ESCRT-III cleaves the buds to generate ILVs when incubated with giant unilamellar vesicles in vitro (Wollert et al., 2009; Wollert and Hurley, 2010). Assembly of ESCRT-II with VPS-20 specifically on the highly curved membrane of endosomes may be required for nucleating ESCRT-III filaments on endosomes to generate ILVs.

In the early embryo CAV-1 and RME-2 on the cell surface are normally internalized and degraded shortly after fertilization (Sato et al., 2006, Balklava et al., 2007). The loss of the ESCRT-I or ESCRT-III-associated protein ALX-1 leads to a significant delay in the degradation of internalized cell surface proteins such as CAV-1 and RME-2 in embryos and the degradation of LIN-12 in the larval vulval precursor cell P6.p (Shaye and Greenwald, 2005; Audhya et al., 2007b; Shi et al., 2007). Depletion of ESCRT-I components results in the loss of VPS-32 from the endosomal membrane, suggesting that ESCRT-I is required for the recruitment of ESCRT-III to the endosomal membrane (Michelet et al., 2009). In the absence of VPS-4, the ESCRT-I and ESCRT-III components over-accumulate on endosomal membranes, suggesting that the requirement for VPS-4 in ESCRT disassembly and dissociation from endosomal membrane is conserved in C. elegans (Audhya et al., 2007b; Michelet et al., 2010). Consistently, knockdown of vps-4 inhibits the degradation of CAV-1 in the early embryo and LET-23 in the late embryo, and leads to the accumulation of enlarged endosomes (Kobuna et al., 2010). Loss of all four oxysterol-binding protein-related proteins (ORPs) produced similar phenotypes and is additive with vps-4 RNAi (Kobuna et al., 2010). ORPs transfer cholesterol between organellar membranes, suggesting that the endosomal cholesterol is required for normal MVB formation/function (Kobuna et al., 2010).

Recently, it has been shown that K63-linked ubiquitylation is important for the sorting of transmembrane proteins into ILVs in C. elegans (Sato et al., 2014). Ubc13 and the non-catalytic E2 variant Uev1A/Mms2 are known to form a complex and function as the E2 enzyme specifically mediating K63-linked ubiquitylation in yeast and mammals (Eddins et al., 2006). In worm $u b c-13$ or uev-1 mutant embryos, lysosomal degradation of CAV-1 and other membrane proteins is severely impaired. In these mutant embryos, cargo proteins are internalized and accumulate in endosomes as in wild-type embryos, but are abnormally recycled to the PM, suggesting that K63-linked ubiquitylation is required for efficient MVB sorting (Sato et al., 2014). 


\subsection{Lysosomes}

The ultimate destination for cargo that enters the degradative pathway is the lysosome, an organelle packed with digestive enzymes and transporters, capable of breaking down most macromolecules into constituent parts small enough to be transported through the lysosomal membrane into the cytoplasm, providing small molecules that support new macromolecular synthesis and provide energy (Luzio et al., 2007). How cargo reaches the lysosome, and the dynamics of lysosomal membranes, remain important topics of study. Lysosomes are often called terminal compartments, but many studies indicate that lysosomes are dynamic interconnected organelles, and can fuse with the plasma membrane under certain conditions (Blott and Griffiths, 2002).

One prevalent model for late endosome to lysosome transport is that late endosomes fuse with pre-exisiting lysosomes to form hybrid organelles, followed by reformation of lysosomes (Luzio et al., 2007). ARL-8 is a small GTPase of Arf family, which mainly localizes to lysosomes in C. elegans (Nakae et al., 2010). In arl-8 mutants, RAB-7-positive late endosomes, including endocytosed macromolecules, fail to fuse with lysosomes containing the aspartic protease ASP-1 (Nakae et al., 2010). In addition, arl-8 mutants suppress the formation of the enlarged late endosome-lysosome hybrid organelles of cup-5 mutants, indicating that ARL-8 functions upstream of CUP-5 (see CUP-5 description below). ARL-8 is also involved in apoptotic cell removal, mediating fusion of phagosomes with lysosomes after phagocytosis (Sasaki et al., 2013). The number of cell corpses significantly increases in the hermaphrodite gonad of the arl-8 mutant and cell corpses engulfed by the sheath cells accumulate in RAB-7-positive phagosomes. ARL-8 is mainly localized to lysosomes but is also recruited to phagosomes at the late stages of phagosome maturation. This transient recruitment to phagosomes is regulated by RAB-7 and the HOPS complex, both of which mediate phagosome-lysosome fusion (Kinchen et al., 2008; Akbar et al., 2011). VPS-41, a component of the HOPS complex preferentially binds to the GTP-form of ARL-8, suggesting that it functions as one of the effectors of ARL-8 in the phagocytic pathway.

One of the first proteins described affecting coelomocyte endocytic function was CUP-5, a homologue of human mucolipin-1, mutations in which leads to mucolipidosis type IV, a lysosomal storage disease in humans (Fares and Greenwald, 2001b). CUP-5/mucolipin family proteins are thought to act as $\mathrm{Ca}^{2+}$ channels, allowing regulated efflux of $\mathrm{Ca}^{2+}$ from lysosomes. Lysosomal function is strongly impaired in cup-5 mutants, with very poor cargo degradation in many cell types. cup-5 mutants display severe defects in lysosome biogenesis that result in the formation of grossly enlarged vacuoles bearing mixed markers for late endosomes and lysosomes (Treusch et al., 2004). In wild-type animals CUP-5 labels the lysosome limiting membrane (Fares and Greenwald, 2001b).

Current models indicate that CUP-5 mediates hybrid organelle $\mathrm{Ca}^{2+}$ release that is required for the reformation of lysosomes after endosome-lysosome fusion (Treusch et al., 2004). Although initially identified due to its coelomocyte phenotype, CUP-5 protein is broadly expressed and affects lysosome function in many or all cells (Hersh et al., 2002; Schaheen et al., 2006a; Campbell and Fares, 2010; Davis et al., 2010; Sun et al., 2011). The original allele identified in the cup mutant screen is hypomorphic (Fares and Greenwald, 2001b; Hersh et al., 2002). cup-5 null mutants were identified in screens for ced mutants that accumulate apoptotic cell corpses (Hersh et al., 2002). Such null alleles are maternal-effect lethal.

The embryonic lethality of cup-5 mutants has been traced to two separable defects: starvation of embryonic cells that can be rescued by supplementation with the lipid soluble metabolite methylpyruvate, and developmental defects that can be rescued by inactivation of the ABC transporter MRP-4 (Schaheen et al., 2006a; Schaheen et al., 2006b). MRP-4 is an ABC transporter localized to specialized lysosome-related organelles (LROs) of the intestine that is likely to transport lipophilic compounds into these compartments (Schaheen et al., 2006b; Currie et al., 2007; Campbell and Fares, 2010). In cup-5 mutant embryos, MRP-4 grossly over-accumulates in the arrested endosome-lysosome hybrid organelles (Schaheen et al., 2006b). mrp-4 mutants suppress the defects in lysosomal degradation apparent in the cup-5 mutant embryonic intestine and restore most cup-5 null mutant embryos to viability, although many rescued animals are sterile (Schaheen et al., 2006a; Schaheen et al., 2006b). MRP-4 is only expressed in the intestinal cells, and lysosomal function in other tissues of cup-5; mrp-4 double mutants is not restored. These results indicate that overdose or missorting of MRP-4 in the embryonic intestine of cup-5 mutants is the major cause of lysosomal dysfunction in the cup-5 mutant intestine, and is a leading cause of embryonic lethality. It remains to be determined if lysosome function in other tissues of cup-5 mutants depends upon similar over-accumulation of other $\mathrm{ABC}$ transporters.

Another mutant affecting lysosomes is $p p k-3$. ppk-3 hypomorphic mutants are vacuolated with grossly enlarged lysosomes (Nicot et al., 2006). Unlike cup-5 mutants, however, ppk-3 mutants appear to have normal degradative function. In addition, double mutant analysis indicates that PPK-3 functions downstream of CUP-5. PPK-3/FAB-1 is a PI-kinase that converts PI(3)P to PI(3,5)P2. One function ascribed to PPK-3 homologs in other species is in the retrieval of membrane from lysosomes. Thus it was proposed that PPK-3 acts in a late lysosome 
maturation process, after digestion of lysosomal contents, in which vesicular budding removes excess membrane from such lysosomes. The very large lysosomes of ppk-3 mutants appear unacidified based upon labeling with lysotracker, but smaller lysosomes that are acidified remain. Since lysosomal hydrolases require an acid environment for function, and lysosomal degradative function appears normal in $p p k-3$ mutants, it was proposed that the small acidic lysosomes mature into and/or fuse with the large more neutral pH lysosomes (Nicot et al., 2006).

As mentioned above, one important function of lysosomes is to salvage raw materials, such as amino acids, for re-use. Lysosomes are an important source of free amino acids, which require transporters to reach the cytoplasm from the lysosome lumen (Winchester, 2001). Defects in amino acid transporters can lead to lysosomal storage diseases (Platt et al., 2012). One long-sought amino acid transporter was discovered in a $C$. elegans genetic screen designed to identify mutants defective in the degradation of apoptotic corpses. laat-1 mutants were found to have enlarged lysosomes and a general defect in lysosome-mediated degradation, that includes apoptotic corpses, but that also affects many lysosomal substrates, including those that enter lysosomes by endocytosis or autophagy (Liu et al., 2012). LAAT-1 is a lysosome-localized protein with homology to lysosomal cystine transporters. Importantly, laat-1 mutants accumulate lysine and arginine in their lysosomes, and forced plasma membrane expression of LAAT-1 or its mammalian homolog PQLC2 results in a specific increase in arginine and lysine import in cultured cells (Liu et al., 2012).

laat-1 mutants displayed very slow embryonic growth, and this growth defect could be rescued by supplementing the animals with high levels of free lysine and arginine (Liu et al., 2012). These results suggest that normal embryonic growth requires lysosome derived lysine and arginine. The LYS/ARG treatment did not rescue the lysosome size or degradative capacity of laat-1 mutant lysosomes. It may be that high-level accumulation of lysine and arginine disrupts lysosome function. laat- 1 mutants are synthetic lethal with $g c n-2$ mutants, consistent with the idea that laat- 1 mutants can only survive if the the amino acid response (AAR) pathway, a repressor of global protein synthesis, is active. FRAP experiments with cytoplasmically expressed mCherry supported the idea that protein synthesis is slowed in laat-1 mutants and is restored upon supplementation with lysine and arginine.

Cystinosis, the accumulation of cystine in the lysosome, is a lysosomal storage disease caused by mutations in ctns-1, which encodes a cystine transporter (Gahl et al., 2002). One treatment for cystinosis is the application of cysteamine, which reacts with accumulated lysosomal cystine, converting cystine to the mixed disulfide of cysteine-cysteamine, which resembles lysine. This conversion is thought to allow export to the cytoplasm of the cysteine-cysteamine mixed disulfide, presumably via a lysine-arginine transporter (Gahl et al., 1985). To test aspects of this model in C. elegans, ctns- 1 mutants (lacking the cystine transporter) were used as a $C$. elegans model for cystinosis (Liu et al., 2012). ctsn-1 mutants accumulated lysosomal cystine and displayed enlarged lysosomes in coelomocytes. The lysosomal enlargement was rescued by treatment with cysteamine, similar to results in cells from cystinosis patients, but laat-1 mutants blocked this rescue of ctns- 1 mutants by cysteamine-treatment (Liu et al., 2012). These results suggest that LAAT-1 is the key lysine transporter that allows this treatment to work.

\subsection{Lysosome-related organelles (LROs)}

In addition to conventional lysosomes, $C$. elegans intestinal cells contain abundant autofluorescent and birefringent lysosome-related organelles (LROs) called gut granules (Clokey and Jacobson, 1986; Hermann et al., 2005). C. elegans gut granules were recently discovered to perform an essential role in zinc storage (Roh et al., 2012). Since gut granules are stained with lysosome-specific fluorescent dyes such as acridine orange and lysotracker, these are thought to be acidified LROs like melanosomes and platelet dense granules in mammals (Clokey and Jacobson, 1986: Hermann et al., 2005). Biogenesis of gut granules requires clathrin-adaptor AP3-components (apt-6, apt-7, vps-16, vps-41) but not AP1 or AP2 components, suggesting that AP3-mediaed lysosomal trafficking is involved in gut granule formation (Hermann et al., 2005).

To study biogenesis of this organelle, several mutants that show a reduction or loss of gut granules were isolated and referred to as the glo (gut granule loss) mutants (Hermann et al., 2005). In the glo- 1 and glo-2 mutant, gut granules are lost at the bean-stage of embryogenesis, apparently by fusion with the apical intestinal membrane, dumping the autofluorescent and birefringent material into the lumen. The glo-3 phenotype is similar, but some gut granules are retained. Notably, the activity and distribution of the conventional endocytic pathway appears largely unaffected in these mutants (Hermann et al., 2005; Rabbitts et al., 2008). glo-1 encodes a Rab GTPase homologous to mammalian Rab38, a protein involved in the biogenesis of the mammalian LROs such as melanosomes and platelet dense granules (Loftus et al., 2002; Oiso et al., 2004). Likewise the Drosophila glo-1 homolog Rab-RP1/Lightnoid is required for another type of LRO, the pigment granules found in retinal pigment cells (Fujikawa et al., 2002; Ma et al., 2004). C. elegans GLO-1 is highly expressed in the intestine and localizes to the gut granules (Hermann et al., 2005). C. elegans GLO-4 is a homolog of Drosophila Claret, which is a putative 
nucleotide exchange factor of Drosophila Rab-RP1 (Hermann et al., 2005). glo-1 and glo-4 are epistatic to mutations in AP3 components. glo- 1 and glo-4 are also expressed in neurons and required for RPM-1-mediated axon termination and synaptogenesis possibly by regulating the formation or accumulation of late endosomes in neurons (Grill et al., 2007).

ABC transporter PGP-2 was identified as GLO-5, and mainly localizes to gut granules in intestinal cells (Schroeder et al., 2007). pgp-2 mutants display reduced numbers of gut granules in intestinal cells, and mislocalize autofluorescent and birefringent gut granule contents to the gut lumen. In pgp-2 mutants, like glo-1 and apm-3/apt-7 mutants, the acidification of gut granules appears impaired. Since most ABC transporters act to transport substrates unidirectionally across membranes, PGP-2 may regulate gut granule biogenesis by modulating membrane composition, possibly via lipid transfer across organellar membranes. glo-3 encodes a novel worm-specific membrane protein, which is expressed in the intestine and localizes to gut granules (Rabbitts et al., 2008). By epistasis analysis with the other glo mutants, GLO-3 appears to function in parallel or downstream of AP3 and PGP-2 in gut granule biogenesis, although its precise role is unknown.

glo-2 encodes a pallidin/HSP-9 homologue, which is a subunit of the BLOC-1 complex (Hermann et al., 2012). GLO-2::GFP driven by the own promoter was expressed in the intestinal cells and localized in the cytoplasm. In mammals AP3, BLOC-1, BLOC-2 and BLOC-3 are all protein complexes involved in the biogenesis of LROs such as melanosomes and platelet dense granules. Mutations in the components of these complexes are associated with Hermansky-Pudlak syndrome (Huizing et al., 2008; Cullinane et al., 2011; Sitaram et al., 2012). The C. elegans genome encodes 7 BLOC-1 subunits including GLO-2, BLOS-1, BLOS-2, BLOS-4, DSBN-1, MUTD-1, and SNPN-1, but seems to lack a BLOS-3 homolog (Hermann et al., 2012). In yeast two-hybrid assay GLO-2 interacts with itself, BLOS-1, and BLOS-4, while SNPN-1 interacts with DSBN-1, suggesting that BLOC-1 components form complexes in C. elegans as they do in other species (Hermann et al., 2012). When each BLOC-1 subunit was knocked down in the background of a partial loss of function glo-2(tm592) allele, the Glo phenotype of glo-2(tm592) was enhanced. In addition, snpn-1 null mutants showed a complete loss of gut granules, again suggesting that the BLOC-1 complex functions in the gut granule biogenesis. In wild-type animals, PGP-2 and CDF-2, which is a zinc transporter homologous to ZnT2/ZnT3, localizes to gut granules, while LMP-1 localizes to both gut granules and conventional lysosomes (Schroeder et al., 2007; Davis et al., 2009; Hermann et al., 2012; Roh et al., 2012). In glo-2(zu455) and snpn-1(tm1892) null mutants, PGP-2-positive gut granules disappeared, and LMP-1 and CDF-2::GFP were mislocalized to the plasma membrane and conventional lysosomes, suggesting that BLOC-1 function is important for proper sorting of these proteins to gut granules (Hermann et al., 2012). In AP3 deficient mutants, PGP-2-positive gut granules still remained, albeit in greatly reduced numbers. In these mutants, LMP-1 disappeared from PGP-2-positive gut granules, suggesting that AP3 is essential for LMP-1 targeting to gut granules. Although AP3 is required for ZnT3 trafficking in mammals, AP3 is not essential for targeting of CDF-2::GFP to gut granules in C. elegans. These disparities in phenotype suggest that, although BLOC-1 interacts physically and functionally with AP3 in mammalian cells, its function is probably not limited to interaction with AP3 (Di Pietro et al., 2006).

\section{The exocytic pathway (secretion)}

After initial synthesis in the ER, lumenal proteins and the lumenal domains of transmembrane proteins are subject to chaperone-mediated protein folding and protein complex formation, and initial glycosylation. Proper folding and protein complex formation is monitored by the ER quality control system, which will not allow misfolded or improperly assembled complexes out of the ER, and will eventually degrade them if folding/assembly fails for an extended period. The C. elegans ER can be visualized by a variety of GFP-tagged marker proteins or by immunostaining using antibodies to endogenous ER-resident proteins such as RET-1 (Basham and Rose, 2001; Rolls et al., 2002; Poteryaev et al., 2005; Audhya et al., 2007a). The most widely used marker for ER in living $C$. elegans is GFP-tagged signal peptidase SP12 (C34B2.10) (Rolls et al., 2002; Poteryaev et al., 2005).

Correctly folded proteins are exported from ER exit sites to the Golgi apparatus via COPII coated vesicles. ER exit sites can be identified in C. elegans cells using antibodies against SEC-16 or TFG-1 (Witte et al., 2011). Sec16 is known to function as a scaffold protein for COPII at ER exit sites in yeast and mammals, and is essential for ER-Golgi transport (Miller and Barlowe, 2010). Recent work in C. elegans was the first to identify TFG-1 as a SEC-16 binding protein that regulates the assembly of SEC-16 with COPII at ER exit sites (Witte et al., 2011). This work also showed that TFG-1 is required for ER-exit of several transmembrane proteins in the C. elegans germline, and that TFG-1 is required for secretion in human cells. SEC-16 and TFG-1 colocalize at ER-exit sites and are juxtaposed to Golgi ministacks. Thus it appears that ER-exit-site/Golgi-ministack pairs form functional secretory units in C. elegans. A similar organization is observed in other invertebrates such as Drosophila (Lerner et al., 2013). 
COPII coated vesicle formation is regulated by the small GTPase Sar1, and the COPII coat itself consists of Sec13-Sec31 and Sec23-Sec24 subcomplexes (Dancourt and Barlowe, 2010). A C. elegans sec-23 mutant was identified in a screen for genes affecting secretion of embryonic cuticle collagen DPY-7 by hypodermal cells (Roberts et al., 2003). This work showed that sec-23 is continually required for viability and fertility, and is required for cell surface accumulation of the GLP-1 and RME-2 receptors in the germline. Depletion of COPII subunits by RNAi also blocks the transport of CAV-1::GFP in oocytes as well as YP170::GFP secretion in the intestine (see below), further indicating a wide requirement for COPII in C. elegans ER-Golgi transport, as expected from analysis in yeast and mammals (Sato et al., 2006; Balklava et al., 2007).

In the Golgi apparatus, cargo proteins are subject to further glycosylation, modification, and processing. Golgi-to-ER retrograde transport and intra-Golgi retrograde transport are thought to be mediated by COPI-coated vesicles in yeast and mammalian cells. Formation of the COPI-coated vesicles is regulated by small GTPase ARF1 and the coatomer complex $\left(\alpha, \beta, \beta^{\prime}, \gamma, \delta, \varepsilon, \zeta\right)$, all of which are conserved in C. elegans. RNAi-mediated knockdown of COPI genes causes intracellular accumulation of normally secreted proteins, but these precise functions of COPI in C. elegans have not been studied in detail (Grant and Hirsh, 1999; Balklava et al., 2007).

Early/Medial Golgi cisternae can be visualized by GFP-tagged AMAN-2 (also called MANS/mannosidase) and RER-1 (a homolog of the yeast retrieval receptor for ER membrane proteins) (Sato et al., 2011). GFP-tagged SYN-16 (a syntaxin 16 homolog) and RAB-6.2 mark late-Golgi compartments (Simonsen et al., 1998; Jantsch-Plunger and Glotzer, 1999; Chen et al., 2006; Gengyo-Ando et al., 2007; Zhang et al., 2012a). Most of early Golgi compartments appear directly juxtaposed to late Golgi compartments, indicating that Golgi mini-stacks contain early and late compartments in $C$. elegans, which is similar to mammals but distinct from budding yeast where early and late Golgi compartments are spatially separated (Sato et al., 2011). Depending upon their sorting in the TGN, newly synthesized proteins are transported to the plasma membrane or endosomes. Lysosomal hydrolases are typically sorted in the TGN for delivery to endosomes by mannose-6-phosphate receptors or Vps10/sortillin type receptors in other organisms. However, $C$. elegans does not have any obvious homologs of mannose-6-phosphate receptors or Vps10/sortillin type receptors, leaving it a mystery for the moment as to how the sorting of lysosomal hydrolases is achieved. Interestingly, Golgi compartments are often observed closely juxtaposed to RAB-5-positive endosomes and partly to RME-1 positive tubulo-vesicular recycling endosomes, consistent with the existence of transport pathways linking these organelles (Sato et al., 2009; Kang et al., 2011; Sato et al., 2011).

\subsection{Yolk as a model cargo for studies of secretion and endocytosis}

C. elegans yolk is a lipoprotein particle related to LDL, expressed specifically in the adult hermaphrodite intestine, that is responsible for transporting lipids from the intestine to the oocytes (Kimble and Sharrock, 1983; Sharrock, 1983). Similarly, vertebrate yolk particles, in animals such as birds and amphibians, transport lipids from the liver to the oocytes. C. elegans yolk consists of yolk proteins (YPs) YP170a, YP170b, YP115, and YP88 complexed with phospholipids, cholesterol, triglicerides, and other lipids (Kimble and Sharrock, 1983; Sharrock, 1983; Sharrock et al., 1990). The polyunsaturated fatty acids (PUFAs) found in C. elegans yolk are precursors of eicosanoid signaling molecules required for attracting sperm to oocytes for fertilization (Kubagawa et al., 2006). $C$. elegans YP170 shares significant homology with ApoB-100, a core component of mammalian LDL particles responsible for lipid transport between the liver and peripheral tissues, and LDL-mediated lipid transport is likely to have evolved from the yolk transport system (Nardelli et al., 1987; Shen et al., 1993). Consistent with this idea, yolk receptors such as the C. elegans yolk receptor RME-2 are oocyte-specific member of the LDL receptor superfamily (Sappington and Raikhel, 1998; Grant and Hirsh, 1999; Stifani et al., 1990).

C. elegans YP170 fused to GFP (YP170::GFP) has been used extensively as a marker protein for basolateral secretion by the intestine and endocytosis by the oocytes. Genome-wide RNAi screens identified many proteins required for yolk secretion, including COPII proteins SAR-1, SEC-13 and SEC-23 (Grant and Hirsh, 1999; Balklava et al., 2007). RNAi-mediated knockdown of COPI components, which are involved in retrograde transport from the Golgi to the ER and intra-Golgi transport in yeast and mammalian cells, also results in accumulation of YP170::GFP in the intestinal cells (Grant and Hirsh, 1999; Balklava et al., 2007). These results suggest that yolk is transported in a COPII- and COPI-dependent fashion as expected. SNARE proteins important for yolk secretion include SYN-5, a Syntaxin type t-SNARE required for ER to the Golgi transport, and SNAP-29, a SNAP-25 family t-SNARE required for multiple transport steps (Kang et al., 2011; Sato et al., 2011). Mechanisms of yolk transport from the Golgi to the plasma membrane remain unknown.

\subsection{Regulated secretion of cortical granules in the zygote}

Cortical granules (CGs) are fertilization-regulated secretory granules that deliver enzymes for the modification of the zona pellucida, a process important for the prevention of polyspermy in animal zygotes (Liu, 2011). Although 
CG exocytosis is a classic example of developmentally controlled trafficking, until recently the molecular mechanisms underlying the biogenesis and exocytosis of CGs had remained largely unknown. In C. elegans, CGs were first identified because they are strongly labeled by CAV-1::GFP expressed in oocytes (Sato et al., 2006; Sato et al., 2008a). This work showed that as developing oocytes enter diplotene of meiosis I, CAV-1::GFP begins to accumulate in large vesicles associated with the Golgi. These CAV-1::GFP positive CGs are scattered around the cytoplasm of developing oocytes, but redistribute to a bimodal localization near the plasma membrane and around the nucleus during oocyte maturation and ovulation. Upon fertilization, all CGs move to the cell periphery and appear to be tethered to the PM. Finally, CGs undergo synchronous fusion with the PM. C. elegans CG exocytosis is required for proper eggshell formation and normal cytokinesis of embryonic cells, rather than the prevention of polyspermy (Bembenek et al., 2007; Sato et al., 2008a). Chondroitin synthesis is known to be required for early embryonic cytokinesis, and chondroitin proteoglycans, along with other extracellular matrix components such as mucin-like glycoproteins, are known CG cargo released upon plasma membrane fusion (Mizuguchi et al., 2003; Bembenek et al., 2007; Sato et al., 2008a; Olson et al., 2012).

The small GTPases, SAR-1 and RAB-1, are required for the transport of CAV-1::GFP from the ER to the Golgi. The formation of CGs from the Golgi depends on an Arf family GTPase, ARF-1, and its guanine nucleotide exchange factor, AGEF-1 (Sato et al., 2006). RAB-11, SYN-4 (syntaxin 1 homolog), RAB-6.1, and Separase are all required for late steps CG exocytosis (Bembenek et al., 2007; Sato et al., 2008a; Kimura and Kimura, 2012). SNB-1 (synaptobrevin1 homolog) localizes to CGs and may function in the fusion with the PM together with SYN-4, although this awaits a direct demonstration (Sato et al., 2008a). Interestingly, the localization of RAB-11 seems to be dynamically regulated during oocyte maturation. In growing oocytes, RAB-11 is required for the endocytic recycling of a yolk receptor, RME-2, and GFP::RAB-11 localizes to the peripheral recycling endosomes and the Golgi-like puncta (Grant and Hirsh, 1999). When oocytes mature in response to a signal from sperm, GFP::RAB-11 moves transiently to the CGs (Sato et al., 2008a). After CG exocytosis, GFP::RAB-11 redistributes to endosomes in zygotes.

The timing of CG exocytosis is tightly linked to developmental events. CG exocytosis occurs at the onset of anaphase I after fertilization (Sato et al., 2006). The anaphase promoting complex (APC/C) is essential for the transition from metaphase I to anaphase I (Golden et al., 2000). Depletion of APC/C subunits does not affect CG biogenesis or plasma membrane tethering, but blocks CG exocytosis (Sato et al., 2006). Mutations in separase (SEP-1), an effector of APC/C, also blocks CG exocytosis, and SEP-1 is thought to function directly in CG exocytosis, in addition to its more well-known roles in chromosome segregation (Bembenek et al., 2007). During anaphase separase cleaves a subunit of cohesin, allowing the sister chromatid segregation (Nasmyth, 2002). APC/C activates seperase by degrading securin, an inhibitor of separase.

Work in C. elegans identified a new and unexpected role for seperase in membrane traffic (Bembenek et al., 2007). Transient recruitment of SEP-1 to CGs appears to be required for CG exocytosis. Such recruitment requires cyclin-dependent kinase CDK-1 and the small GTPase, RAB-6.1 (Kimura and Kimura, 2012), and is negatively regulated by protein phosphatase 5 (Richie et al., 2011). RAB-6.1 may directly recruit separase to the cortical granules, but no physical interaction between these two proteins has yet been demonstrated. Interestingly recent work indicates that depletion of separase or securin affects both exocytic and endocytic pathways in mammalian cells by affecting acidification of the endomembrane system (Bacac et al., 2011). Thus separase and securin may have more general roles in membrane trafficking, rather than being specific for CG exocytosis, although their precise role on membranes remains unclear.

\subsection{Secretion of Hedgehog-related proteins}

Hedgehog $(\mathrm{Hh})$ family proteins are secreted signaling proteins with diverse developmental roles in metazoans (Therond, 2012). Canonical Hh proteins are proproteins consisting of an N-terminal and a C-terminal domain. The C-terminal domain contains a protease with autocatalytic cleavage activity. In Drosophila, after Hh has been subject to autocleavage, a cholesterol moiety is covalently linked to the Hh C-terminus, and a palmitoyl moiety is added to its N-terminus. Palmytoylation of $\mathrm{Hh}$ proteins is catalyzed by $\mathrm{O}$-acyltransferase. Secretion of lipid-modified $\mathrm{Hh}$ depends upon Dispatched (Disp), which has 12 transmembrane-domains and a sterol sensing domain (Burke et al., 1999). C. elegans has 61 proteins that show a similarity with the C-terminal protease domain of Hh, although the $C$. elegans genome lacks a direct Hh ortholog (Zugasti et al., 2005). The 61 C. elegans Hh-related proteins have been divided into 4 families named Warthog (WRT), Ground hog (GRD), Ground hog-like (GRL), and Quahog (QUA). The $C$. elegans genome also contains two dispatched homologs, CHE-14 and PTD-2 (see WormBook chapter Homologs of the Hh signaling network in C. elegans). The che-14 gene is expressed in epithelial cells of the ectoderm and localizes to apical membranes (Michaux et al., 2000). che-14 mutants accumulate secretory vesicles beneath the apical surface of the amphid support cells, and amorphous materials at the apical surface of the epidermis, indicating a role for CHE-14 in apical secretion. 
Exosomes are small secreted vesicles released from the cell surface via direct fusion of MVBs, including their ILVs (exosomes), with the plasma membrane (Bobrie et al., 2011). Exosomes appear to be involved in the apical secretion of Hh-related proteins in C. elegans (Liegeois et al., 2006). In wild-type animals, small light MVBs, including ILVs, are often observed just beneath the apical membrane of the hypodermis, while small vesicles, which appear to be derived from ILVs in MVBs, are found immediately above the plasma membrane in the inner cuticular layer, suggesting that exosomes are released from the apical plasma membrane of epidermis by a direct fusion of MVBs with the plasma membrane (Liegeois et al., 2006). Interestingly, some mutant alleles of vha-5, which encodes an a-subunit of the V0 sector functioning together with V1 sectors as the V-ATPase, accumulate large dense MVBs in the hypodermis, and show defects in cuticle formation. The V0 sector of the V-ATPase is known to promote membrane fusion in other organisms and thus may function to help fusion of the MVBs to the apical membrane and release of the internal vesicles as exosomes containing Hh-related proteins (Peters et al., 2001; Bayer et al., 2003; Hiesinger et al., 2005). Consistent with this model, VHA-5 protein localizes to the apical membrane and the limiting membrane of MVBs in the hypodermis (Liegeois et al., 2006). Fluorescently labeled Hh-related proteins such as WRT-2 and WRT-8 accumulate in the MVBs of the epidermis of vha-5 mutant animals, suggesting these may be secreted in a V0 dependent manner. Mutant alleles of vha-5 were also identified as $r d y-1$ (rod-like larval lethality and dye-filling defective), a mutant that displays a che-14-like phenotype (Liegeois et al., 2007). In one mutant that affects the trafficking function of VHA-5, CHE-14 protein accumulates with the mutant VHA-5 protein, presumably in MVBs. These results suggest that VHA-5 is involved in a late step of exosome secretion in the hypodermis. The precise roles of VHA-5 and CHE-14, and the relationship between these proteins in secretion of Hh-related proteins, remain unknown.

\subsection{Secretion of Wnt proteins}

Recent studies in C. elegans have revealed a specific role for the transmembrane protein MIG-14/Wntless in secretion of Wnt proteins such as EGL-20 (Coudreuse et al., 2006; Pan et al., 2008; Yang et al., 2008b). EGL-20 is a Wnt family ligand that directs the migration of the HSN neurons and QL neuroblasts along anterior-posterior axis (see Wnt signaling in C. elegans). Wnt family ligands are lipid-modified secreted proteins that bind to Wnt receptors such as MIG-1 and LIN-17, triggering an intracellular Wnt signaling cascade (Wnt signaling in C. elegans). MIG-14 appears to bind to Wnt ligands in the Golgi and chaperone them to the cell surface for release (Pan et al., 2008; Yang et al., 2008b). Without MIG-14, Wnt-ligands are trapped intracellularly and Wnt secretion is very poor, leading to multiple Wnt-related phenotypes. MIG-14 must be efficiently endocytosed and recycled to maintain this pathway. The recycling process is discussed above.

\subsection{Function of Golgi clathrin and the AP1 clathrin adaptor complex in intestinal tubulogenesis}

Besides endocytosis, another important role for clathrin, with its Golgi adapter AP1, is in the production of vesicles that bud from the Golgi. In general such vesicles are not thought to carry cargo to the plasma membrane, but rather they are thought to carry cargo to endosomes. In most cases these vesicles contain newly synthesized lysosomal hydrolases that will move from endosomes to lysosomes. However in polarized epithelial cells, there is evidence that endosomes can act as intermediaries in the secretory pathway, receiving cargo from the Golgi and sending it to either the basolateral or apical plasma membrane (Ang et al., 2004; Hua et al., 2006). Consistent with this idea, analysis in C. elegans identified a requirement for CHC-1 and the AP1 adaptor in establishing/maintaining polarity during intestinal development (Shafaq-Zadah et al., 2012; Zhang et al., 2012b). Loss of CHC-1 or AP1 subunits mislocalized apical membrane markers basolaterally, and mislocalized some basolateral markers apically. Furthermore loss of CHC-1 or AP1 often produced ectopic lateral lumens. AP1 was also important for the apical localization of peripheral membrane proteins CDC-42 and PAR-6. Thus it appears that AP1 is important for the apico-basal sorting of secretory proteins in the intestine, and is a key factor in polarized epithelial tubulogenesis. $\mathrm{CHC}$ and AP-1 likely function directly in the sorting process, since work in mammalian polarized MDCK cells shows that knockdown of AP1 components also causes missorting of basolateral proteins (Gonzalez and Rodriguez-Boulan, 2009). This MDCK cell work showed roles for AP1 at the TGN and common recycling endosomes.

\subsection{Secretion of fibroblast growth factor (FGF) EGL-17}

During larval development FGF-type ligand EGL-17 is secreted by the vulval precursor cell P6.p to attract migrating sex myoblast cells (SMs). The SMs migrate anteriorly to the presumptive vulval region, eventually forming the vulval muscles required for efficient egg laying and contributing to the uterus as well (Thomas et al., 1990). Loss of the large lipoprotein receptors LRP-1 and LRP-2 inhibited secretion of EGL-17 from P6.p, and a specific region of the extracellular domain of LRP-2 was shown to bind to EGL-17 in vitro (Kamikura and Cooper, 2003; Kamikura and Cooper, 2006). Thus it was proposed that LRP-1 and LRP-2 bind to EGL-17 in the ER or Golgi 
and chaperone EGL-17 through the secretory system to the cell surface for release. The clathrin adapter DAB-1 was shown to bind to the intracellular domains of LRP-1 and LRP-2, and dab-1 mutants trap EGL-17 in the VPCs, blocking EGL-17 secretion (Kamikura and Cooper, 2003; Kamikura and Cooper, 2006). Loss of DAB-1 may affect EGL-17 indirectly by trapping LRP-1 and LRP-2 at the plasma membrane, or directly, by blocking secretion from the Golgi. EGL-17 secretion also depended upon the clathrin adapter complex AP-1, UNC-41/stonin and APT-9/gga1 clathrin adapters, and GTPases RAB-5 and RAB-8, suggesting that EGL-17/LRP complex secretion requires transport through endosomes.

\subsection{COG and GARP tethering complexes}

The COG (conserved oligomeric Golgi) complex consists of 8 subunits (COG1-COG8) and has been shown to function as a vesicle tethering factor that regulates retrograde transport within the Golgi and between the Golgi and endosome-derived transport vesicles (Miller and Ungar, 2012). In other species loss of COG function leads to mislocalization of Golgi glycosylation enzymes and defects in Golgi-mediated glycosylation of secreted proteins (Miller and Ungar, 2012). The C. elegans genome encodes homologs of all 8 COG complex subunits (Kubota et al., 2006). Recently, mutant alleles of $\operatorname{cog} c-1$ and $\operatorname{cog} c-3$, which encode $C$. elegans COG1 and COG3 respectively, were identified as mig mutants defective in the migration of gonadal distal tip cells (DTCs) (Kubota et al., 2006). Further investigation showed that a Golgi-localized nucleocide diphosphatase, MIG-23, is mislocalized, and its steady-state levels dramatically reduced in the $\operatorname{cog} c$ mutants.

Gonadal DTCs migration is regulated by several secreted proteins such as UNC-6/netrin and the ADAM family proteases, MIG-17 and GON-1. In the $\operatorname{cog} c$ mutants, MIG-17 glycosylation is incomplete. Such underglycosylated MIG-17 is secreted, but fails to properly assemble within the gonadal basement membrane where it is required for gonad migration (Kubota et al., 2006). These observations suggests that normal glycosylation of guidance factors in the Golgi is important for the correct targeting of these molecules to a specific ECM destinations.

The GARP (Golgi-associated retrograde transport) complex consists of 4 subunits (Vps51p, Vps52p, Vps53, Vps54p) and has been shown to function as a vesicle-tethering factor for the retrograde transport from endosomes to the Golgi in other species. The C. elegans genome has genes encoding all 4 GARP subunits (Luo et al., 2011). The C. elegans GARP complex localizes to the Golgi and interacts with Golgi SNAREs, such as SYN-5, SYN-16, and VTI-1, as well as the small GTPases, RAB-6.1 and RAB-6.2, as seen in yeast and mammals. Knockout mutants of each GARP subunit gene are viable but exhibit enlargement of lysosomal compartments in coelomocytes. Combination of the vps-52 mutation with a mutation of the COG complex genes leads to a synthetic lethal phenotype, suggesting that the GARP complex shares partly overlapping functions with the COG complex at the Golgi. It remains to be determined if COG or GARP affect endosome to Golgi transport of cargo in C. elegans.

\section{Autophagy in C. elegans embryos}

Macroautophagy (hereafter called autophagy) is a transport pathway leading to lysosomes (Mizushima, 2011). In autophagy, cytosolic proteins and organelles are sequestered by double-membrane autophagosomes. Autophagosomes then fuse with lysosomes, resulting in degradation of their contents. The bulk degradation of cytoplasm by autophagy is essential for cell survival under starvation conditions, promoting the recycling of cellular materials such as amino acids and lipids. In addition to bulk degradation, autophagy could be a selective process, which mediates the degradation of specific targets such as pathogens, protein aggregates, and organelles. Many proteins, including ATG proteins, have been identified as regulators of autophagy in yeast and mammals, and most of them are conserved in C. elegans (see WormBook chapter Autophagy in C. elegans). In C. elegans, autophagy is involved in various physiological processes including dauer formation (Melendez et al., 2003), survival of L1 larvae under starvation condition (Kang et al., 2007), longevity (Melendez et al., 2003; Hars et al., 2007; Jia and Levine, 2007; Hansen et al., 2008; Toth et al., 2008; Lapierre et al., 2011) and necrotic-like degeneration of neurons (Samara et al., 2008; Toth et al., 2008). Autophagy genes also play a role in apoptotic cell corpse degradation during development (Ruck et al., 2011; Gong et al., 2012; Li et al., 2012). More information about this work is summarized in detail in Autophagy in C. elegans.

In addition to these functions, recent studies have revealed that autophagy also plays unique physiological roles during early embryogenesis. ATG8/LC3 generally serves as a marker of the autophagosomal membrane in other systems, and the worm has two ATG8/LC3 homologs, LGG-1 and LGG-2 (Melendez et al., 2003). When autophagic activity is monitored in embryos, autophagosomes labeled with GFP::LGG1/2 surround the penetrating paternal pronucleus in the fertilized 1-cell-stage embryos (Al Rawi et al., 2011; Sato and Sato, 2011). In addition to the 1-cell stage embryos, puncta of GFP::LGG-1 are induced in whole embryos around the 32- to 64-cell stages, suggesting multiple rounds of induction of autophagy during embryogenesis (Sato and Sato, 2011). The first 
induction of autophagy in 1-cell stage embryos depends on fertilization, because autophagy is not induced in unfertilized eggs of the spe- 9 mutant that has infertile sperm but shows normal oocyte maturation and ovulation. Interestingly, in polyspermic embryos autophagosomes surround each penetrating sperm. This observation suggests that autophagy is induced by sperm entry to remove some of the paternally provided components. In fact, it was determined that these autophagosomes selectively sequester paternally inherited organelles such as mitochondria and membranous organelles (MOs), and target them to lysosomal degradation (Al Rawi et al., 2011; Sato and Sato, 2011). MOs are sperm-specific post-Golgi organelles essential for sperm fertility and enter the ooplasm upon fertilization together with sperm mitochondria (Gleason et al., 2006). This type of autophagy is referred to as "allophagy" for allogeneic (non-self) organelle autophagy (Sato and Sato, 2012). Allophagy could also explain the mechanism of maternal inheritance of mitochondrial DNA (mtDNA). Maternal (uniparental) inheritance of mtDNA is observed in many species, although the precise mechanism has not been clear (Sato and Sato, 2013b). mtDNA is maternally inherited in wild-type $C$. elegans. However, in autophagy-defective mutants, paternal mtDNA is abnormally transmitted to the next generation, suggesting that paternal mtDNA is eliminated from embryos by allophagy (Al Rawi et al., 2011; Sato and Sato, 2011). More work will be required to understand how the paternal organelles are recognized and targeted to autophagosomes. Ubiquitylation may be involved in MO recognition because a strong ubiquitylation signal is detectable on MOs in fertilized embryos. In addition to autophagy, it has been reported that RNAi of some proteasomal subunits results in the defective elimination of paternal mtDNA from embryos (Zhou et al., 2011b), implying that the proteasomal system is also involved in degradation of paternal mitochondria.

Autophagy is also involved in degradation of extra P-granule components in embryonic somatic cells. P-granules are large ribonucleoprotein complexes found specifically in the germ line cytoplasm, and PGL-1 and PGL-3 proteins are representative constituents of P-granules (Kawasaki et al., 1998; Kawasaki et al., 2004; Updike and Strome, 2010). During early embryogenesis, several mechanisms promote segregation of maternally inherited P-granules specifically to the germ cell lineage. Autophagy further degrades extra P-granule components in embryonic somatic cells. In 100-cell stage embryos defective in autophagy, PGL-1 and PGL-3 remain abnormally in somatic cells and form aggregates called PGL granules. sepa- 1 was identified in a genetic screen for suppressors of the ectopic accumulation of PGL granules in autophagy mutants (Zhang et al., 2009). It was demonstrated that SEPA-1 binds both PGL-3 and LGG-1 and functions as an adaptor molecule that recruits P-granule components to autophagosomes. Many additional autophagy-related mutants were identified in a worm genetic screen based on ectopic accumulation of GFP::PGL-1 in somatic cells (epg: ectopic PGL granule mutants). This screen successfully identified several genes conserved in mammals but not in yeast, suggesting that worm genetics is a powerful tool to identify new factors conserved in multicellular organisms (Lu et al., 2013 ; Sato and Sato, 2013a).

\section{Summary}

Recent years have marked an explosion in membrane trafficking data from C. elegans studies. Many of these studies have identified novel mechanisms that regulate specific transport steps and have shown that the mechanistic insights gleaned from analysis of trafficking in C. elegans can predict the mechanisms of membrane trafficking in mammals. Many studies have started to integrate this basic cell biological information with analysis of higher order development and physiology. Understanding how trafficking regulates, and is regulated by, the development and functions of differing cell-types and tissues will remain a major goal for the field to tackle in the coming years.

\section{Tables 1 and 2}

Table 1: Arf, Sar, and Rab family genes

\begin{tabular}{|l|l|l|l|}
\hline Arf/Sar family & Gene number & Chrom & Human homolog \\
\hline Gene name & F45E4.1 & IV & Arf1 \\
\hline arf-1.1/arl-6 & B0336.2 & III & Arf1 \\
\hline arf-1.2/arf-1 & F57H12.1 & IV & Arf5 \\
\hline arf-3 & Y116A8C.12 & IV & Arf6 \\
\hline arf-6 & F54C9.10 & II & Arl1 \\
\hline arl-1 & F22B5.1 & II & Arl2 \\
\hline arf-2/evl-20/arl-2/arl-5 & F19H8.3 & II & Arl3 \\
\hline arl-3 & & 27 & \\
\hline
\end{tabular}


C. elegans as a model for membrane traffic

\begin{tabular}{|c|c|c|c|}
\hline arl-5/arl-2 & ZK632.8 & III & Arl-5 \\
\hline arl-6/bbs-3 & C38D4.8 & III & Arl6 \\
\hline arl-8 & Y57G11C.13 & IV & Arl8 \\
\hline arl-13 & Y37E3.5 & I & Arl13 \\
\hline sar-1 & ZK180.4 & IV & Sar1 \\
\hline \multicolumn{4}{|l|}{ Rab family } \\
\hline Gene name & Gene number & Chrom & Human \\
\hline$r a b-1$ & C39F7.4 & V & Rab1a \\
\hline rab-2/unc-108/unc-67 & F53F10.4 & I & Rab2 \\
\hline$r a b-3 / r b l-3$ & C18A3.6 & II & Rab3 \\
\hline$r a b-5$ & F26H9.6 & I & Rab5 \\
\hline rab-6.1 & F59B2.7 & III & Rab6 \\
\hline$r a b-6.2$ & T25G12.4 & $X$ & Rab6 \\
\hline rab-7 & W03C9.3 & II & Rab7 \\
\hline$r a b-8$ & \begin{tabular}{|l|} 
D1037.4 \\
\end{tabular} & I & Rab8 \\
\hline$r a b-10$ & $\mathrm{~T} 23 \mathrm{H} 2.5$ & I & Rab10 \\
\hline rab-11.1 & F53G12.1 & I & Rab11 \\
\hline$r a b-11.2$ & W04G5.2 & I & Rab11 \\
\hline$r a b-14$ & K09A9.2 & $\mathrm{X}$ & Rab14 \\
\hline rab-18/pra-1 & Y92C3B.3 & III & Rab18 \\
\hline$r a b-19$ & Y62E10A.9 & IV & Rab43 \\
\hline$r a b-21$ & T01B7.3 & II & Rab21 \\
\hline rab-27/aex-6 & Y87G2A.4 & I & Rab27 \\
\hline$r a b-28$ & Y11D7A.4 & IV & Rab28 \\
\hline$r a b-30$ & Y45F3A.2 & III & Rab30 \\
\hline glo-1 & R07B1.12 & $\mathrm{x}$ & Rab32 \\
\hline$r a b-33$ & \begin{tabular}{|l|} 
F43D9.2 \\
\end{tabular} & III & Rab33 \\
\hline rab-35/rme-5 & Y47D3A.25 & III & Rab35 \\
\hline$r a b-37$ & W01H2.3 & $\mathrm{X}$ & Rab37 \\
\hline$r a b-39$ & D2013.1 & II & Rab39 \\
\hline RabY1/tag-312 & C33D12.6 & $\mathrm{X}$ & $\begin{array}{l}\text { Ras and EF-hand } \\
\text { domain-containing protein }\end{array}$ \\
\hline rabY2 & 4R79.2 & IV & $\begin{array}{l}\text { EF-hand calcium-binding } \\
\text { domain-containing protein } \\
\text { 4B }\end{array}$ \\
\hline rabY3 & K02E10.1 & $\mathrm{X}$ & Rab1 \\
\hline rabY4 & F11A5.4 & $\mathrm{V}$ & Rab2 \\
\hline rabY5 & F11A5.3 & $\mathrm{V}$ & Rab2 \\
\hline rabY6 & C56E6.2 & II & Rab6 \\
\hline N/A & Y71H2AM.12 & III & Rab6 \\
\hline N/A & ZK669.5 & II & Rab23 \\
\hline
\end{tabular}


Table 2: SNARE family genes

\begin{tabular}{|c|c|c|c|}
\hline \multicolumn{4}{|l|}{ Syntaxin family } \\
\hline Gene name & Gene number & Chrom & Human \\
\hline unc-64/syx-1 & F56A8.7a,b & III & Syntaxin 1A \\
\hline syn-2/syx-2 & F48F7.2 & $\mathrm{X}$ & Syntaxin 1A \\
\hline syn-1/syx-3 & F35C8.4 & $\mathrm{X}$ & Syntaxin 1B \\
\hline syn-4/syx-4 & T01B11.3 & IV & Syntaxin 1B \\
\hline syn-3/syx-5 & F55A11.2 & V & Syntaxin 5 \\
\hline syx-6 & C15C7.1 & $\mathrm{X}$ & Syntaxin 6 \\
\hline syn-13/syx-7 & F36F2.4 & I & Syntaxin 12 \\
\hline syn-16 & ZC155.7 & III & Syntaxin 16 \\
\hline syx-17 & VF39H2L.1 & I & Syntaxin 17 \\
\hline syx-18 & T10H9.3 & $\mathrm{V}$ & Syntaxin 18 \\
\hline \multicolumn{4}{|l|}{ SNAP25 family } \\
\hline Gene name & Gene number & Chrom & Human \\
\hline ric-4/snap-25 & Y22F5A.3 & $\mathrm{V}$ & SNAP-25 \\
\hline aex-4 & T14G12.2 & $\mathrm{X}$ & SNAP-25 \\
\hline snap-29 & K02D10.5 & III & SNAP-29 \\
\hline \multicolumn{4}{|l|}{ Other SNAREs } \\
\hline Gene name & Gene number & Chrom & Human \\
\hline snb-1/sup-8 & T10H9.4 & V & VAMP2 \\
\hline snb-2 & F23H12.1 & V & VAMP2 \\
\hline snb-7 & ZK795.4 & IV & VAMP3 \\
\hline snb-5 & C30A5.5 & III & VAMP3 \\
\hline snb-6 & T14D7.3 & II & VAMP3 \\
\hline N/A & B0513.9 & IV & VAMP8 \\
\hline N/A & Y69A2AR.6 & IV & VAMP8 \\
\hline vti-1 & Y57G11C.4 & IV & Vti1 \\
\hline nbet-1 & Y59E9AL.7 & IV & BET1 \\
\hline memb-1/gosr-2.1 & B0272.2 & $\mathrm{X}$ & $\begin{array}{l}\text { Golgi SNAP receptor } \\
\text { complex member } 2\end{array}$ \\
\hline memb-2 & M03E7.5 & $\mathrm{V}$ & $\begin{array}{l}\text { Golgi SNAP receptor } \\
\text { complex member } 2\end{array}$ \\
\hline gos-28/gosr-1 & F08F8.8 & III & $\begin{array}{l}\text { Golgi SNAP receptor } \\
\text { complex member } 1\end{array}$ \\
\hline$y k t-6$ & B0361.10 & III & YKT6 \\
\hline sec-22 & F55A4.1 & $\mathrm{X}$ & Sec22 \\
\hline sec-20 & F40G9.1 & III & BNIP1 \\
\hline use-1 & Y110A7A.11 & I & USE1 \\
\hline
\end{tabular}




\section{Acknowledgements}

We thank Willisa Liou (Department of Anatomy, Chang Gung University, Taiwan, ROC) for the anti-RME-1 immunoEM image in Figure 6. This work was supported by NIH Grants GM067237 and GM103995 to B.D.G. and F32 GM096599 to A.N., by the Funding Program for Next Generation World-leading Researchers (NEXT program), The Naito Foundation, The Mochida Memorial Foundation for Medical and Pharmaceutical Research, and The Sumitomo Foundation to K.S., and by JSPS KAKENHI Grant Number 23687027 to M.S.

\section{References}

Akbar, M.A., Tracy, C., Kahr, W.H., and Kramer, H. (2011). The full-of-bacteria gene is required for phagosome maturation during immune defense in Drosophila. J. Cell Biol. 192, 383-390. Abstract Article

Al Rawi, S., Louvet-Vallee, S., Djeddi, A., Sachse, M., Culetto, E., Hajjar, C., Boyd, L., Legouis, R., and Galy, V. (2011). Postfertilization autophagy of sperm organelles prevents paternal mitochondrial DNA transmission. Science 334, 1144-1147. Abstract Article

Allaire, P.D., Marat, A.L., Dall'Armi, C., Di Paolo, G., McPherson, P.S., and Ritter, B. (2010). The Connecdenn DENN domain: a GEF for Rab35 mediating cargo-specific exit from early endosomes. Mol. Cell 37, $370-382$. Abstract Article

Ang, A.L., Folsch, H., Koivisto, U.M., Pypaert, M., and Mellman, I. (2003). The Rab8 GTPase selectively regulates AP-1B-dependent basolateral transport in polarized Madin-Darby canine kidney cells. J. Cell Biol. 163, 339-350. Abstract Article

Ang, A.L., Taguchi, T., Francis, S., Folsch, H., Murrells, L.J., Pypaert, M., Warren, G., and Mellman, I. (2004). Recycling endosomes can serve as intermediates during transport from the Golgi to the plasma membrane of MDCK cells. J. Cell Biol. 167, 531-543. Abstract

Ang, S.F., and Folsch, H. (2012). The role of secretory and endocytic pathways in the maintenance of cell polarity. Essays Biochem. 53, 29-39. Abstract Article

Audhya, A., Desai, A., and Oegema, K. (2007a). A role for Rab5 in structuring the endoplasmic reticulum. J. Cell Biol. 178, 43-56. Abstract Article

Audhya, A., McLeod, I.X., Yates, J.R., and Oegema, K. (2007b). MVB-12, a fourth subunit of metazoan ESCRT-I, functions in receptor downregulation. PLoS One 2, e956. Abstract Article

Babbey, C.M., Ahktar, N., Wang, E., Chen, C.C., Grant, B.D., and Dunn, K.W. (2006). Rab10 regulates membrane transport through early endosomes of polarized Madin-Darby canine kidney cells. Mol. Biol. Cell 17, 3156-3175. Abstract

Bacac, M., Fusco, C., Planche, A., Santodomingo, J., Demaurex, N., Leemann-Zakaryan, R., Provero, P., and Stamenkovic, I. (2011). Securin and separase modulate membrane traffic by affecting endosomal acidification. Traffic 12, 615-626. Abstract Article

Balklava, Z., Pant, S., Fares, H., and Grant, B.D. (2007). Genome-wide analysis identifies a general requirement for polarity proteins in endocytic traffic. Nat. Cell Biol. 9, 1066-1073. Abstract Article

Basham, S.E., and Rose, L.S. (2001). The Caenorhabditis elegans polarity gene ooc-5 encodes a Torsin-related protein of the AAA ATPase superfamily. Development 128, 4645-4656. Abstract

Bayer, M.J., Reese, C., Buhler, S., Peters, C., and Mayer, A. (2003). Vacuole membrane fusion: V0 functions after trans-SNARE pairing and is coupled to the $\mathrm{Ca}^{2+}$-releasing channel. J. Cell Biol. 162, 211-222. Abstract

Bednarek, E.M., Schaheen, L., Gaubatz, J., Jorgensen, E.M., and Fares, H. (2007). The plasma membrane calcium ATPase MCA-3 is required for clathrin-mediated endocytosis in scavenger cells of Caenorhabditis elegans. Traffic 8, 543-553. Abstract Article 
Belenkaya, T.Y., Wu, Y., Tang, X., Zhou, B., Cheng, L., Sharma, Y.V., Yan, D., Selva, E.M., and Lin, X. (2008). The retromer complex influences Wnt secretion by recycling wntless from endosomes to the trans-Golgi network. Dev. Cell 14, 120-131. Abstract Article

Bembenek, J.N., Richie, C.T., Squirrell, J.M., Campbell, J.M., Eliceiri, K.W., Poteryaev, D., Spang, A., Golden, A., and White, J.G. (2007). Cortical granule exocytosis in C. elegans is regulated by cell cycle components including separase. Development 134, 3837-3848. Abstract Article

Blott, E.J., and Griffiths, G.M. (2002). Secretory lysosomes. Nat. Rev. Mol. Cell Biol. 3, 122-131. Abstract Article

Bobrie, A., Colombo, M., Raposo, G., and Thery, C. (2011). Exosome secretion: molecular mechanisms and roles in immune responses. Traffic 12, 1659-1668. Abstract Article

Bonifacino, J.S., and Glick, B.S. (2004). The mechanisms of vesicle budding and fusion. Cell 116, 153-166. Abstract Article

Brini, M., and Carafoli, E. (2011). The plasma membrane $\mathrm{Ca}^{2+}$ ATPase and the plasma membrane sodium calcium exchanger cooperate in the regulation of cell calcium. Cold Spring Harb. Perspect. Biol. 3, a004168. Abstract Article

Brodsky, F.M., Chen, C.Y., Knuehl, C., Towler, M.C., and Wakeham, D.E. (2001). Biological basket weaving: formation and function of clathrin-coated vesicles. Annu. Rev. Cell Dev. Biol. 17, 517-568. Abstract Article

Brown, F.D., Rozelle, A.L., Yin, H.L., Balla, T., and Donaldson, J.G. (2001). Phosphatidylinositol 4,5-bisphosphate and Arf6-regulated membrane traffic. J. Cell Biol. 154, 1007-1017. Abstract Article

Bucci, C., Parton, R.G., Mather, I.H., Stunnenberg, H., Simons, K., Hoflack, B., and Zerial, M. (1992). The small GTPase rab5 functions as a regulatory factor in the early endocytic pathway. Cell 70, 715-728. Abstract Article

Burbea, M., Dreier, L., Dittman, J.S., Grunwald, M.E., and Kaplan, J.M. (2002). Ubiquitin and AP180 regulate the abundance of GLR-1 glutamate receptors at postsynaptic elements in C. elegans. Neuron 35, 107-120. Abstract Article

Burglin, T.R., and Kuwabara, P.E. (2006). Homologs of the Hh signalling network in C. elegans, WormBook, ed. The C. elegans Research Community, WormBook, doi/10.1895/wormbook.1.76.1, http://www.wormbook.org.

Burke, R., Nellen, D., Bellotto, M., Hafen, E., Senti, K.A., Dickson, B.J., and Basler, K. (1999). Dispatched, a novel sterol-sensing domain protein dedicated to the release of cholesterol-modified hedgehog from signaling cells. Cell 99, 803-815. Abstract Article

Campbell, E.M., and Fares, H. (2010). Roles of CUP-5, the Caenorhabditis elegans orthologue of human TRPML1, in lysosome and gut granule biogenesis. BMC Cell Biol. 11, 40. Abstract Article

Caplan, S., Naslavsky, N., Hartnell, L.M., Lodge, R., Polishchuk, R.S., Donaldson, J.G., and Bonifacino, J.S. (2002). A tubular EHD1-containing compartment involved in the recycling of major histocompatibility complex class I molecules to the plasma membrane. EMBO J. 21, 2557-2567. Abstract Article

Carney, D.S., Davies, B.A., and Horazdovsky, B.F. (2006). Vps9 domain-containing proteins: activators of Rab5 GTPases from yeast to neurons. Trends Cell Biol. 16, 27-35. Abstract Article

Chang, H.C., Hull, M., and Mellman, I. (2004). The J-domain protein Rme-8 interacts with Hsc70 to control clathrin-dependent endocytosis in Drosophila. J. Cell Biol. 164, 1055-1064. Abstract Article

Chavrier, P., and Goud, B. (1999). The role of ARF and Rab GTPases in membrane transport. Curr. Opin. Cell Biol. 11, 466-475. Abstract Article

Chen, B., Jiang, Y., Zeng, S., Yan, J., Li, X., Zhang, Y., Zou, W., and Wang, X. (2010). Endocytic sorting and recycling require membrane phosphatidylserine asymmetry maintained by TAT-1/CHAT-1. PLoS Genet. 6, e1001235. Abstract Article 
Chen, C., Garcia-Santos, D., Ishikawa, Y., Seguin, A., Li, L., Fegan, K.H., Hildick-Smith, G.J., Shah, D.I., Cooney, J.D., Chen, W., et al. (2013). Snx3 regulates recycling of the transferrin receptor and iron assimilation. Cell Metab. 17, 343-352. Abstract Article

Chen, C.C., Schweinsberg, P.J., Vashist, S., Mareiniss, D.P., Lambie, E.J., and Grant, B.D. (2006). RAB-10 is required for endocytic recycling in the Caenorhabditis elegans intestine. Mol. Biol. Cell 17, 1286-1297. Abstract Article

Chen, D., Xiao, H., Zhang, K., Wang, B., Gao, Z., Jian, Y., Qi, X., Sun, J., Miao, L., and Yang, C. (2010). Retromer is required for apoptotic cell clearance by phagocytic receptor recycling. Science 327, 1261-1264. Abstract Article

Cheng, H., Govindan, J.A., and Greenstein, D. (2008). Regulated trafficking of the MSP/Eph receptor during oocyte meiotic maturation in C. elegans. Curr. Biol. 18, 705-714. Abstract Article

Chibalina, M.V., Seaman, M.N., Miller, C.C., Kendrick-Jones, J., and Buss, F. (2007). Myosin VI and its interacting protein LMTK2 regulate tubule formation and transport to the endocytic recycling compartment. J. Cell Sci. 120, 4278-4288. Abstract Article

Chotard, L., Mishra, A.K., Sylvain, M.A., Tuck, S., Lambright, D.G., and Rocheleau, C.E. (2010a). TBC-2 regulates RAB-5/RAB-7-mediated endosomal trafficking in Caenorhabditis elegans. Mol. Biol. Cell 21, 2285-2296. Abstract Article

Chotard, L., Skorobogata, O., Sylvain, M.A., Shrivastava, S., and Rocheleau, C.E. (2010b). TBC-2 is required for embryonic yolk protein storage and larval survival during L1 diapause in Caenorhabditis elegans. PLoS One 5, e15662. Abstract Article

Chun, D.K., McEwen, J.M., Burbea, M., and Kaplan, J.M. (2008). UNC-108/Rab2 regulates postendocytic trafficking in Caenorhabditis elegans. Mol. Biol. Cell 19, 2682-2695. Abstract Article

Clokey, G.V., and Jacobson, L.A. (1986). The autofluorescent "lipofuscin granules" in the intestinal cells of Caenorhabditis elegans are secondary lysosomes. Mech. Ageing Dev. 35, 79-94. Abstract Article

Coudreuse, D.Y., Roel, G., Betist, M.C., Destree, O., and Korswagen, H.C. (2006). Wnt gradient formation requires retromer function in Wnt-producing cells. Science 312, 921-924. Abstract Article

Cullen, P.J., and Korswagen, H.C. (2011). Sorting nexins provide diversity for retromer-dependent trafficking events. Nat. Cell Biol. 14, 29-37. Abstract Article

Cullinane, A.R., Curry, J.A., Carmona-Rivera, C., Summers, C.G., Ciccone, C., Cardillo, N. D., Dorward, H., Hess, R.A., White, J.G., Adams, D., et al. (2011). A BLOC-1 mutation screen reveals that PLDN is mutated in Hermansky-Pudlak Syndrome type 9. Am. J. Hum. Genet. 88, 778-787. Abstract Article

Currie, E., King, B., Lawrenson, A.L., Schroeder, L.K., Kershner, A.M., and Hermann, G.J. (2007). Role of the Caenorhabditis elegans multidrug resistance gene, $m r p-4$, in gut granule differentiation. Genetics 177, 1569-1582. Abstract Article

Dancourt, J., and Barlowe, C. (2010). Protein sorting receptors in the early secretory pathway. Annu. Rev. Biochem. 79, 777-802. Abstract Article

Dang, H., Klokk, T.I., Schaheen, B., McLaughlin, B.M., Thomas, A.J., Durns, T.A., Bitler, B.G., Sandvig, K., and Fares, H. (2011). Derlin-dependent retrograde transport from endosomes to the Golgi apparatus. Traffic 12, 1417-1431. Abstract Article

Dang, H., Li, Z., Skolnik, E.Y., and Fares, H. (2004). Disease-related myotubularins function in endocytic traffic in Caenorhabditis elegans. Mol. Biol. Cell 15, 189-196. Abstract Article

Darland-Ransom, M., Wang, X., Sun, C.L., Mapes, J., Gengyo-Ando, K., Mitani, S., and Xue, D. (2008). Role of $C$. elegans TAT-1 protein in maintaining plasma membrane phosphatidylserine asymmetry. Science 320, 528-531. Abstract Article 
Daumke, O., Lundmark, R., Vallis, Y., Martens, S., Butler, P.J., and McMahon, H.T. (2007). Architectural and mechanistic insights into an EHD ATPase involved in membrane remodelling. Nature 449, 923-927. Abstract Article

Davis, D.E., Roh, H.C., Deshmukh, K., Bruinsma, J.J., Schneider, D.L., Guthrie, J., Robertson, J.D., and Kornfeld, K. (2009). The cation diffusion facilitator gene $c d f-2$ mediates zinc metabolism in Caenorhabditis elegans. Genetics 182, 1015-1033. Abstract Article

Davis, K.M., Sturt, B.L., Friedmann, A.J., Richmond, J.E., Bessereau, J.L., Grant, B.D., and Bamber, B.A. (2010). Regulated lysosomal trafficking as a mechanism for regulating GABAA receptor abundance at synapses in Caenorhabditis elegans. Mol. Cell. Neurosci. 44, 307-317. Abstract Article

Delic, M., Valli, M., Graf, A.B., Pfeffer, M., Mattanovich, D., and Gasser, B. (2013). The secretory pathway: exploring yeast diversity. FEMS Microbiol. Rev. 37, 872-914. Abstract Article

Di Paolo, G., and De Camilli, P. (2006). Phosphoinositides in cell regulation and membrane dynamics. Nature 443, 651-657. Abstract Article

Di Pietro, S.M., Falcon-Perez, J.M., Tenza, D., Setty, S.R., Marks, M.S., Raposo, G., and Dell'Angelica, E.C. (2006). BLOC-1 interacts with BLOC-2 and the AP-3 complex to facilitate protein trafficking on endosomes. Mol. Biol. Cell 17, 4027-4038. Abstract Article

DiNitto, J.P., and Lambright, D.G. (2006). Membrane and juxtamembrane targeting by PH and PTB domains. Biochim. Biophys. Acta 1761, 850-867. Abstract Article

Doherty, G.J., and McMahon, H.T. (2009). Mechanisms of endocytosis. Annu. Rev. Biochem. 78, 857-902. Abstract Article

Doray, B., Lee, I., Knisely, J., Bu, G., and Kornfeld, S. (2007). The $\gamma / \sigma 1$ and $\alpha / \sigma 2$ hemicomplexes of clathrin adaptors AP-1 and AP-2 harbor the dileucine recognition site. Mol. Biol. Cell 18, 1887-1896. Abstract Article

Dwivedi, M., Sung, H., Shen, H., Park, B.J., and Lee, S. (2011). Disruption of endocytic pathway regulatory genes activates autophagy in C. elegans. Mol Cells 31, 477-481. Abstract Article

Eaton, S. (2008). Retromer retrieves wntless. Dev. Cell 14, 4-6. Abstract Article

Eddins, M.J., Carlile, C.M., Gomez, K.M., Pickart, C.M. and Wolberger, C. (2006). Mms2-Ubc13 covalently bound to ubiquitin reveals the structural basis of linkage-specific polyubiquitin chain formation. Nat. Struct. Mol. Biol. 13, 915-920. Abstract Article

Eisenberg, E., and Greene, L.E. (2007). Multiple roles of auxilin and hsc70 in clathrin-mediated endocytosis. Traffic 8, 640-646. Abstract Article

Fares, H., and Greenwald, I. (2001a). Genetic analysis of endocytosis in Caenorhabditis elegans: coelomocyte uptake defective mutants. Genetics 159, 133-145. Abstract

Fares, H., and Greenwald, I. (2001b). Regulation of endocytosis by CUP-5, the Caenorhabditis elegans mucolipin-1 homolog. Nat. Genet. 28, 64-68. Abstract Article

Franch-Marro, X., Wendler, F., Guidato, S., Griffith, J., Baena-Lopez, A., Itasaki, N., Maurice, M.M., and Vincent, J.-P. (2008). Wingless secretion requires endosome-to-Golgi retrieval of Wntless/Evi/Sprinter by the retromer complex. Nat. Cell Biol. 10, 170-177. Abstract Article

Fu, L., Rab, A., Tang, L.P., Rowe, S.M., Bebok, Z., and Collawn, J.F. (2012). Dab2 is a key regulator of endocytosis and post-endocytic trafficking of the cystic fibrosis transmembrane conductance regulator. Biochem. J. 441, 633-643. Abstract Article

Fujikawa, K., Satoh, A.K., Kawamura, S., and Ozaki, K. (2002). Molecular and functional characterization of a unique Rab protein, RABRP1, containing the WDIAGQE sequence in a GTPase motif. Zoolog. Sci. 19, 981-993. Abstract Article 
Funderburk, S.F., Wang, Q.J., and Yue, Z. (2010). The Beclin 1-VPS34 complex-at the crossroads of autophagy and beyond. Trends Cell Biol. 20, 355-362. Abstract Article

Fyfe, I., Schuh, A.L., Edwardson, J.M., and Audhya, A. (2011). Association of the endosomal sorting complex ESCRT-II with the Vps20 subunit of ESCRT-III generates a curvature-sensitive complex capable of nucleating ESCRT-III filaments. J. Biol. Chem. 286, 34262-34270. Abstract Article

Gahl, W.A., Thoene, J.G., and Schneider, J.A. (2002). Cystinosis. N. Engl. J. Med. 347, 111-121. Abstract Article

Gahl, W.A., Tietze, F., Butler, J.D., and Schulman, J.D. (1985). Cysteamine depletes cystinotic leucocyte granular fractions of cystine by the mechanism of disulphide interchange. Biochem. J. 228, 545-550. Abstract

Gallegos, M.E., Balakrishnan, S., Chandramouli, P., Arora, S., Azameera, A., Babushekar, A., Bargoma, E., Bokhari, A., Chava, S.K., Das, P., et al. (2012). The C. elegans Rab family: identification, classification and toolkit construction. PLoS One 7, e49387. Abstract Article

Gengyo-Ando, K., Kuroyanagi, H., Kobayashi, T., Murate, M., Fujimoto, K., Okabe, S., and Mitani, S. (2007). The SM protein VPS-45 is required for RAB-5-dependent endocytic transport in Caenorhabditis elegans. EMBO Rep. 8, 152-157. Abstract Article

Georgiou, M., Marinari, E., Burden, J., and Baum, B. (2008). Cdc42, Par6, and aPKC regulate Arp2/3-mediated endocytosis to control local adherens junction stability. Curr. Biol. 18, 1631-1638. Abstract Article

Girard, M., Poupon, V., Blondeau, F., and McPherson, P.S. (2005). The DnaJ-domain protein RME-8 functions in endosomal trafficking. J. Biol. Chem. 280, 40135-40143. Abstract Article

Gleason, E.J., Lindsey, W.C., Kroft, T.L., Singson, A.W., and L'Hernault S,W. (2006). spe-10 encodes a DHHC-CRD zinc-finger membrane protein required for endoplasmic reticulum/Golgi membrane morphogenesis during Caenorhabditis elegans spermatogenesis. Genetics 172, 145-158. Abstract Article

Glodowski, D.R., Chen, C.C., Schaefer, H., Grant, B.D., and Rongo, C. (2007). RAB-10 regulates glutamate receptor recycling in a cholesterol-dependent endocytosis pathway. Mol. Biol. Cell 18, 4387-4396. Abstract Article

Glodowski, D.R., Wright, T., Martinowich, K., Chang, H.C., Beach, D., and Rongo, C. (2005). Distinct LIN-10 domains are required for its neuronal function, its epithelial function, and its synaptic localization. Mol. Biol. Cell 16, 1417-1426. Abstract Article

Golden, A., Sadler, P.L., Wallenfang, M.R., Schumacher, J.M., Hamill, D.R., Bates, G., Bowerman, B., Seydoux, G., and Shakes, D.C. (2000). Metaphase to anaphase (mat) transition-defective mutants in Caenorhabditis elegans. J. Cell Biol. 151, 1469-1482. Abstract Article

Goldstein, B., and Macara, I.G. (2007). The PAR proteins: fundamental players in animal cell polarization. Dev. Cell 13, 609-622. Abstract Article

Gong, K., Chen, C., Zhan, Y., Chen, Y., Huang, Z., and Li, W. (2012). Autophagy-related gene 7 (ATG7) and reactive oxygen species/extracellular signal-regulated kinase regulate tetrandrine-induced autophagy in human hepatocellular carcinoma. J. Biol. Chem. 287, 35576-35588. Abstract Article

Gonzalez, A., and Rodriguez-Boulan, E. (2009). Clathrin and AP1B: key roles in basolateral trafficking through trans-endosomal routes. FEBS Lett. 583, 3784-3795. Abstract Article

Grant, B., and Hirsh, D. (1999). Receptor-mediated endocytosis in the Caenorhabditis elegans oocyte. Mol. Biol. Cell 10, 4311-4326. Abstract Article

Grant, B.D., and Donaldson, J.G. (2009). Pathways and mechanisms of endocytic recycling. Nat. Rev. Mol. Cell Biol. 10, 597-608. Abstract Article

Grant, B. D. and Sato, M. (2006). Intracellular trafficking, WormBook, ed. The C. elegans Research Community, WormBook, doi/10.1895/wormbook.1.77.1, http://www.wormbook.org. 
Grant, B.D., Zhang, Y., Paupard, M.C., Lin, S.X., Hall, D.H., and Hirsh, D. (2001). Evidence that RME-1, a conserved C. elegans EH-domain protein, functions in endocytic recycling. Nat. Cell Biol. 3, 573-579. Abstract Article

Grill, B., Bienvenut, W.V., Brown, H.M., Ackley, B.D., Quadroni, M., and Jin, Y. (2007). C. elegans RPM-1 regulates axon termination and synaptogenesis through the Rab GEF GLO-4 and the Rab GTPase GLO-1. Neuron 55, 587-601. Abstract Article

Gu, M., Liu, Q., Watanabe, S., Sun, L., Hollopeter, G., Grant, B.D., and Jorgensen, E.M. (2013). AP2 hemicomplexes contribute independently to synaptic vesicle endocytosis. Elife 2, e00190. Abstract Article

Guo, P., Hu, T., Zhang, J., Jiang, S., and Wang, X. (2010). Sequential action of Caenorhabditis elegans Rab GTPases regulates phagolysosome formation during apoptotic cell degradation. Proc. Natl. Acad. Sci. U. S. A. 107, 18016-18021. Abstract Article

Hansen, M., Chandra, A., Mitic, L.L., Onken, B., Driscoll, M., and Kenyon, C. (2008). A role for autophagy in the extension of lifespan by dietary restriction in C. elegans. PLoS Genet. 4, e24. Abstract Article

Hars, E.S., Qi, H., Ryazanov, A.G., Jin, S., Cai, L., Hu, C., and Liu, L.F. (2007). Autophagy regulates ageing in $C$. elegans. Autophagy 3, 93-95. Abstract

Harterink, M., Port, F., Lorenowicz, M.J., McGough, I.J., Silhankova, M., Betist, M.C., van Weering, J.R., van Heesbeen, R.G., Middelkoop, T.C., Basler, K., et al. (2011). A SNX3-dependent retromer pathway mediates retrograde transport of the Wnt sorting receptor Wntless and is required for Wnt secretion. Nat. Cell Biol. 13, 914-923. Abstract Article

Hausmann, G., Banziger, C., and Basler, K. (2007). Helping Wingless take flight: how WNT proteins are secreted. Nat. Rev. Mol. Cell Biol. 8, 331-336. Abstract Article

Henne, W.M., Buchkovich, N.J., and Emr, S.D. (2011). The ESCRT pathway. Dev. Cell 21, 77-91. Abstract Article

Hermann, G.J., Scavarda, E., Weis, A.M., Saxton, D.S., Thomas, L.L., Salesky, R., Somhegyi, H., Curtin, T.P., Barrett, A., Foster, O.K., et al. (2012). C. elegans BLOC-1 functions in trafficking to lysosome-related gut granules. PLoS One 7, e43043. Abstract Article

Hermann, G.J., Schroeder, L.K., Hieb, C.A., Kershner, A.M., Rabbitts, B.M., Fonarev, P., Grant, B.D., and Priess, J.R. (2005). Genetic analysis of lysosomal trafficking in Caenorhabditis elegans. Mol. Biol. Cell 16, 3273-3288. Abstract Article

Hersh, B.M., Hartwieg, E., and Horvitz, H.R. (2002). The Caenorhabditis elegans mucolipin-like gene cup-5 is essential for viability and regulates lysosomes in multiple cell types. Proc. Natl. Acad. Sci. U. S. A. 99, 4355-4360. Abstract

Hiesinger, P.R., Fayyazuddin, A., Mehta, S.Q., Rosenmund, T., Schulze, K.L., Zhai, R.G., Verstreken, P., Cao, Y., Zhou, Y., Kunz, J., and Bellen, H.J. (2005). The v-ATPase V0 subunit a1 is required for a late step in synaptic vesicle exocytosis in Drosophila. Cell 121, 607-620. Abstract Article

Hoekstra, D., Tyteca, D., and van IJizendoorn, S.C.D. (2004). The subapical compartment: a traffic center in membrane polarity development. J. Cell Sci. 117, 2183-2192. Abstract Article

Holmes, A., Flett, A., Coudreuse, D., Korswagen, H.C., and Pettitt, J. (2007). C. elegans Disabled is required for cell-type specific endocytosis and is essential in animals lacking the AP-3 adaptor complex. J. Cell Sci. 120, 2741-2751. Abstract Article

Horiuchi, H., Giner, A., Hoflack, B., and Zerial, M. (1995). A GDP/GTP exchange-stimulatory activity for the Rab5-RabGDI complex on clathrin-coated vesicles from bovine brain. J. Biol. Chem. 270, 11257-11262. Abstract

Hua, W., Sheff, D., Toomre, D., and Mellman, I. (2006). Vectorial insertion of apical and basolateral membrane proteins in polarized epithelial cells revealed by quantitative 3D live cell imaging. J. Cell Biol. 172, 1035-1044. Abstract Article 
Huizing, M., Helip-Wooley, A., Westbroek, W., Gunay-Aygun, M., and Gahl, W.A. (2008). Disorders of lysosome-related organelle biogenesis: clinical and molecular genetics. Annu. Rev. Genomics Hum. Genet. 9, 359-386. Abstract Article

Hurley, J.H. (2010). The ESCRT complexes. Crit. Rev. Biochem. Mol. Biol. 45, 463-487. Abstract Article

Hutagalung, A.H., and Novick, P.J. (2011). Role of Rab GTPases in membrane traffic and cell physiology. Physiol. Rev. 91, 119-149. Abstract Article

Hyenne, V., Tremblay-Boudreault, T., Velmurugan, R., Grant, B.D., Loerke, D., and Labbe, J.C. (2012). RAB-5 controls the cortical organization and dynamics of PAR proteins to maintain C. elegans early embryonic polarity. PLoS One 7, e35286. Abstract Article

Jahn, R., and Scheller, R.H. (2006). SNAREs-engines for membrane fusion. Nat. Rev. Mol. Cell Biol. 7, 631-643. Abstract

Jantsch-Plunger, V., and Glotzer, M. (1999). Depletion of syntaxins in the early Caenorhabditis elegans embryo reveals a role for membrane fusion events in cytokinesis. Curr. Biol. 9, 738-745. Abstract Article

Jia, K., and Levine, B. (2007). Autophagy is required for dietary restriction-mediated life span extension in $C$. elegans. Autophagy 3,597-599. Abstract

Jovic, M., Kieken, F., Naslavsky, N., Sorgen, P.L., and Caplan, S. (2009). Eps15 homology domain 1-associated tubules contain phosphatidylinositol-4-phosphate and phosphatidylinositol-(4,5)-bisphosphate and are required for efficient recycling. Mol. Biol. Cell 20,2731-2743. Abstract Article

Kaksonen, M., Toret, C.P., and Drubin, D.G. (2006). Harnessing actin dynamics for clathrin-mediated endocytosis. Nat. Rev. Mol. Cell Biol. 7, 404-414. Abstract Article

Kamikura, D.M., and Cooper, J.A. (2003). Lipoprotein receptors and a disabled family cytoplasmic adaptor protein regulate EGL-17/FGF export in C. elegans. Genes Dev. 17, 2798-2811. Abstract Article

Kamikura, D.M., and Cooper, J.A. (2006). Clathrin interaction and subcellular localization of Ce-DAB-1, an adaptor for protein secretion in Caenorhabditis elegans. Traffic 7, 324-336. Abstract Article

Kanamori, T., Inoue, T., Sakamoto, T., Gengyo-Ando, K., Tsujimoto, M., Mitani, S., Sawa, H., Aoki, J., and Arai, H. (2008). $\beta$-Catenin asymmetry is regulated by PLA1 and retrograde traffic in C. elegans stem cell divisions. EMBO J. 27, 1647-1657. Abstract Article

Kang, C., You, Y.J., and Avery, L. (2007). Dual roles of autophagy in the survival of Caenorhabditis elegans during starvation. Genes Dev. 21, 2161-2171. Abstract Article

Kang, J., Bai, Z., Zegarek, M.H., Grant, B.D., and Lee, J. (2011). Essential roles of snap-29 in C. elegans. Dev. Biol. 355, 77-88. Abstract Article

Kang, Y.L., Yochem, J., Bell, L., Sorensen, E.B., Chen, L., and Conner, S.D. (2013). Caenorhabditis elegans reveals a FxNPxY-independent low-density lipoprotein receptor internalization mechanism mediated by epsin 1. Mol. Biol. Cell 24, 308-318. Abstract Article

Katzmann, D.J., Odorizzi, G., and Emr, S.D. (2002). Receptor downregulation and multivesicular-body sorting. Nat. Rev. Mol. Cell Biol. 3, 893-905. Abstract Article

Kawasaki, I., Amiri, A., Fan, Y., Meyer, N., Dunkelbarger, S., Motohashi, T., Karashima, T., Bossinger, O., and Strome, S. (2004). The PGL family proteins associate with germ granules and function redundantly in Caenorhabditis elegans germline development. Genetics 167, 645-661. Abstract Article

Kawasaki, I., Shim, Y.H., Kirchner, J., Kaminker, J., Wood, W.B., and Strome, S. (1998). PGL-1, a predicted RNA-binding component of germ granules, is essential for fertility in C. elegans. Cell 94, 635-645. Abstract Article 
Kimble, J., and Sharrock, W.J. (1983). Tissue-specific synthesis of yolk proteins in Caenorhabditis elegans. Dev. Biol. 96, 189-196. Abstract Article

Kimura, K., and Kimura, A. (2012). Rab6 is required for the exocytosis of cortical granules and the recruitment of separase to the granules during the oocyte-to-embryo transition in Caenorhabditis elegans. J. Cell Sci. 125, 5897-5905. Abstract Article

Kinchen, J.M., Doukoumetzidis, K., Almendinger, J., Stergiou, L., Tosello-Trampont, A., Sifri, C.D., Hengartner, M.O., and Ravichandran, K.S. (2008). A pathway for phagosome maturation during engulfment of apoptotic cells. Nat. Cell Biol. 10, 556-566. Abstract Article

Kinchen, J.M., and Ravichandran, K.S. (2010). Identification of two evolutionarily conserved genes regulating processing of engulfed apoptotic cells. Nature 464, 778-782. Abstract Article

Kobuna, H., Inoue, T., Shibata, M., Gengyo-Ando, K., Yamamoto, A., Mitani, S., and Arai, H. (2010). Multivesicular body formation requires OSBP-related proteins and cholesterol. PLoS Genet. 6, e100105. Abstract Article

Korolchuk, V.I., Schütz, M.M., Gómez-Llorente, C., Rocha, J., Lansu, N.R., Collins, S.M., Wairkar, Y.P., Robinson, I.M., and O'Kane, C.J. (2007). Drosophila Vps35 function is necessary for normal endocytic trafficking and actin cytoskeleton organisation. J. Cell Sci. 120, 4367-4376. Abstract Article

Kroschewski, R., Hall, A., and Mellman, I. (1999). Cdc42 controls secretory and endocytic transport to the basolateral plasma membrane of MDCK cells. Nat. Cell Biol. 1, 8-13. Abstract Article

Kubagawa, H.M., Watts, J.L., Corrigan, C., Edmonds, J.W., Sztul, E., Browse, J., and Miller, M.A. (2006). Oocyte signals derived from polyunsaturated fatty acids control sperm recruitment in vivo. Nat. Cell Biol. 8, 1143-1148. Abstract Article

Kubota, Y., Sano, M., Goda, S., Suzuki, N., and Nishiwaki, K. (2006). The conserved oligomeric Golgi complex acts in organ morphogenesis via glycosylation of an ADAM protease in C. elegans. Development 133, 263-273. Abstract Article

Lapierre, L.R., Gelino, S., Melendez, A., and Hansen, M. (2011). Autophagy and lipid metabolism coordinately modulate life span in germline-less C. elegans. Curr. Biol. 21, 1507-1514. Abstract Article

Lauwers, E., Jacob, C., and Andre, B. (2009). K63-linked ubiquitin chains as a specific signal for protein sorting into the multivesicular body pathway. J. Cell Biol. 185, 493-502. Abstract Article

Lee, D.W., Zhao, X., Scarselletta, S., Schweinsberg, P.J., Eisenberg, E., Grant, B.D., and Greene, L.E. (2005). ATP binding regulates oligomerization and endosome association of RME-1 family proteins. J. Biol. Chem. 280, 17213-17220. Abstract Article

Leibfried, A., Fricke, R., Morgan, M.J., Bogdan, S., and Bellaiche, Y. (2008). Drosophila Cip4 and WASp define a branch of the Cdc42-Par6-aPKC pathway regulating E-cadherin endocytosis. Curr. Biol. 18, 1639-1648. Abstract Article

Lerner, D.W., McCoy, D., Isabella, A.J., Mahowald, A.P., Gerlach, G.F., Chaudhry, T.A., and Horne-Badovinac, S. (2013). A Rab10-dependent mechanism for polarized basement membrane secretion during organ morphogenesis. Dev. Cell 24, 159-168. Abstract Article

Li, W., Zou, W., Yang, Y., Chai, Y., Chen, B., Cheng, S., Tian, D., Wang, X., Vale, R.D., and Ou, G. (2012). Autophagy genes function sequentially to promote apoptotic cell corpse degradation in the engulfing cell. J. Cell Biol. 197, 27-35. Abstract Article

Li, W., Zou, W., Zhao, D., Yan, J., Zhu, Z., Lu, J., and Wang, X. (2009). C. elegans Rab GTPase activating protein TBC-2 promotes cell corpse degradation by regulating the small GTPase RAB-5. Development 136, 2445-2455. Abstract Article 
Li, X., Chen, B., Yoshina, S., Cai, T., Yang, F., Mitani, S., and Wang, X. (2013). Inactivation of Caenorhabditis elegans aminopeptidase DNPP-1 restores endocytic sorting and recycling in tat-1 mutants. Mol. Biol. Cell 24, 1163-1175. Abstract Article

Liegeois, S., Benedetto, A., Garnier, J.M., Schwab, Y., and Labouesse, M. (2006). The V0-ATPase mediates apical secretion of exosomes containing Hedgehog-related proteins in Caenorhabditis elegans. J. Cell Biol. 173, 949-961. Abstract Article

Liegeois, S., Benedetto, A., Michaux, G., Belliard, G., and Labouesse, M. (2007). Genes required for osmoregulation and apical secretion in Caenorhabditis elegans. Genetics 175, 709-724. Abstract Article

Lin, S.X., Grant, B., Hirsh, D., and Maxfield, F.R. (2001). Rme-1 regulates the distribution and function of the endocytic recycling compartment in mammalian cells. Nat. Cell Biol. 3, 567-572. Abstract Article

Lindmo, K., and Stenmark, H. (2006). Regulation of membrane traffic by phosphoinositide 3-kinases. J. Cell Sci. 119, 605-614. Abstract Article

Liu, B., Du, H., Rutkowski, R., Gartner, A., and Wang, X. (2012). LAAT-1 is the lysosomal lysine/arginine transporter that maintains amino acid homeostasis. Science 337, 351-354. Abstract Article

Liu, M. (2011). The biology and dynamics of mammalian cortical granules. Reprod. Biol. Endocrinol. 9, 149. Abstract Article

Loftus, S.K., Larson, D.M., Baxter, L.L., Antonellis, A., Chen, Y., Wu, X., Jiang, Y., Bittner, M., Hammer, J.A., 3rd, and Pavan, W.J. (2002). Mutation of melanosome protein RAB38 in chocolate mice. Proc. Natl. Acad. Sci. U. S. A. 99, 4471-4476. Abstract Article

Lu, N., Shen, Q., Mahoney, T.R., Liu, X., and Zhou, Z. (2011). Three sorting nexins drive the degradation of apoptotic cells in response to PtdIns(3)P signaling. Mol. Biol. Cell 22, 354-374. Abstract Article

Lu, N., and Zhou, Z. (2012). Membrane trafficking and phagosome maturation during the clearance of apoptotic cells. Int. Rev. Cell Mol. Biol. 293, 269-309. Abstract Article

Lu, Q., Wu, F., and Zhang, H. (2013). Aggrephagy: lessons from C. elegans. Biochem. J. 452, 381-390. Abstract Article

Luo, L., Hannemann, M., Koenig, S., Hegermann, J., Ailion, M., Cho, M.K., Sasidharan, N., Zweckstetter, M., Rensing, S.A., and Eimer, S. (2011). The Caenorhabditis elegans GARP complex contains the conserved Vps51 subunit and is required to maintain lysosomal morphology. Mol. Biol. Cell 22, 2564-2578. Abstract Article

Luzio, J.P., Pryor, P.R., and Bright, N.A. (2007). Lysosomes: fusion and function. Nat. Rev. Mol. Cell Biol. 8, 622-632. Abstract Article

Ma, J., Plesken, H., Treisman, J.E., Edelman-Novemsky, I., and Ren, M. (2004). Lightoid and Claret: a rab GTPase and its putative guanine nucleotide exchange factor in biogenesis of Drosophila eye pigment granules. Proc. Natl. Acad. Sci. U. S. A. 101, 11652-11657. Abstract Article

Maxfield, F.R., and McGraw, T.E. (2004). Endocytic recycling. Nat. Rev. Mol. Cell Biol. 5, 121-132. Abstract Article

Mayers, J.R., Fyfe, I., Schuh, A.L., Chapman, E.R., Edwardson, J.M., and Audhya, A. (2011). ESCRT-0 assembles as a heterotetrameric complex on membranes and binds multiple ubiquitinylated cargoes simultaneously. J. Biol. Chem. 286, 9636-9645. Abstract Article

Mayers, J.R., Wang, L., Pramanik, J., Johnson, A., Sarkeshik, A., Wang, Y., Saengsawang, W., Yates J.R. 3rd, and Audhya, A. (2013). Regulation of ubiquitin-dependent cargo sorting by multiple plasma membrane endocytic adaptor proteins. Proc. Natl. Acad. Sci. U. S. A. 110, 11857-11862. Abstract Article

Mayor, S., and Pagano, R.E. (2007). Pathways of clathrin-independent endocytosis. Nat. Rev. Mol. Cell Biol. 8, 603-612. Abstract Article 
McGhee, J.D. (2007). The C. elegans intestine, WormBook, ed. The C. elegans Research Community, WormBook, doi/10.1895/wormbook.1.133.1, http://www.wormbook.org

McGough, I.J., and Cullen, P.J. (2013). Clathrin is not required for SNX-BAR-retromer-mediated carrier formation. J. Cell Sci. 126, 45-52. Abstract Article

Meléndez, A. and Levine, B. (2009). Autophagy in C. elegans, WormBook, ed. The C. elegans Research Community, WormBook, doi/10.1895/wormbook.1.147.1, http://www.wormbook.org.

Melendez, A., Talloczy, Z., Seaman, M., Eskelinen, E.L., Hall, D.H., and Levine, B. (2003). Autophagy genes are essential for dauer development and life-span extension in C. elegans. Science 301, 1387-1391. Abstract Article

Michaux, G., Gansmuller, A., Hindelang, C., and Labouesse, M. (2000). CHE-14, a protein with a sterol-sensing domain, is required for apical sorting in C. elegans ectodermal epithelial cells. Curr. Biol. 10, 1098-1107. Abstract Article

Michelet, X., Alberti, A., Benkemoun, L., Roudier, N., Lefebvre, C., and Legouis, R. (2009). The ESCRT-III protein CeVPS-32 is enriched in domains distinct from CeVPS-27 and CeVPS-23 at the endosomal membrane of epithelial cells. Biol. Cell 101, 599-615. Abstract Article

Michelet, X., Djeddi, A., and Legouis, R. (2010). Developmental and cellular functions of the ESCRT machinery in pluricellular organisms. Biol. Cell 102, 191-202. Abstract Article

Miller, E.A., and Barlowe, C. (2010). Regulation of coat assembly-sorting things out at the ER. Curr. Opin. Cell Biol. 22, 447-453. Abstract Article

Miller, V.J., and Ungar, D. (2012). Re'COG'nition at the Golgi. Traffic 13, 891-897. Abstract Article

Mishra, S.K., Keyel, P.A., Hawryluk, M.J., Agostinelli, NR., Watkins, S.C., and Traub, L.M. (2002). Disabled-2 exhibits the properties of a cargo-selective endocytic clathrin adaptor. EMBO J. 21, 4915-4926. Abstract Article

Mizuguchi, S., Uyama, T., Kitagawa, H., Nomura, K.H., Dejima, K., Gengyo-Ando, K., Mitani, S., Sugahara, K., and Nomura, K. (2003). Chondroitin proteoglycans are involved in cell division of Caenorhabditis elegans. Nature 423, 443-448. Abstract Article

Mizushima, N. (2011). Autophagy in protein and organelle turnover. Cold Spring Harb. Symp. Quant. Biol. 76, 397-402. Abstract Article

Mukherjee, S., Ghosh, R.N., and Maxfield, F.R. (1997). Endocytosis. Physiol. Rev. 77, 759-803. Abstract

Musch, A., Cohen, D., Kreitzer, G., and Rodriguez-Boulan, E. (2001). cdc42 regulates the exit of apical and basolateral proteins from the trans-Golgi network. EMBO J. 20, 2171-2179. Abstract Article

Nakae, I., Fujino, T., Kobayashi, T., Sasaki, A., Kikko, Y., Fukuyama, M., Gengyo-Ando, K., Mitani, S., Kontani, K., and Katada, T. (2010). The arf-like GTPase Arl8 mediates delivery of endocytosed macromolecules to lysosomes in Caenorhabditis elegans. Mol. Biol. Cell 21, 2434-2442. Abstract Article

Nakayama, Y., Shivas, J.M., Poole, D.S., Squirrell, J.M., Kulkoski, J.M., Schleede, J.B., and Skop, A.R. (2009). Dynamin participates in the maintenance of anterior polarity in the Caenorhabditis elegans embryo. Dev. Cell 16, 889-900. Abstract Article

Nardelli, D., Gerber-Huber, S., van het Schip, F.D., Gruber, M., Geert, A.B., and Wahli, W. (1987). Vertebrate and nematode genes coding for yolk proteins are derived from a common ancestor. Biochemistry 26, 6397-6402. Abstract Article

Naslavsky, N., Weigert, R., and Donaldson, J.G. (2003). Convergence of non-clathrin- and clathrin-derived endosomes involves Arf6 inactivation and changes in phosphoinositides. Mol. Biol. Cell 14, 417-431. Abstract Article 
Naslavsky, N., Weigert, R., and Donaldson, J.G. (2004). Characterization of a nonclathrin endocytic pathway: membrane cargo and lipid requirements. Mol. Biol. Cell 15, 3542-3552. Abstract Article

Nasmyth, K. (2002). Segregating sister genomes: the molecular biology of chromosome separation. Science 297, 559-565. Abstract Article

Nicot, A.S., Fares, H., Payrastre, B., Chisholm, A.D., Labouesse, M., and Laporte, J. (2006). The phosphoinositide kinase PIKfyve/Fablp regulates terminal lysosome maturation in Caenorhabditis elegans. Mol. Biol. Cell 17, 3062-3074. Abstract Article

Nilsson, L., Conradt, B., Ruaud, A.F., Chen, C.C., Hatzold, J., Bessereau, J.L., Grant, B.D., and Tuck, S. (2008). Caenorhabditis elegans num-1 negatively regulates endocytic recycling. Genetics 179, 375-387. Abstract Article

Nilsson, L., Jonsson, E., and Tuck, S. (2011). Caenorhabditis elegans Numb inhibits endocytic recycling by binding TAT-1 aminophospholipid translocase. Traffic 12, 1839-1849. Abstract Article

Nordmann, M., Cabrera, M., Perz, A., Brocker, C., Ostrowicz, C., Engelbrecht-Vandre, S., and Ungermann, C. (2010). The Mon1-Ccz1 complex is the GEF of the late endosomal Rab7 homolog Ypt7. Curr. Biol. 20, 1654-1659. Abstract Article

Oikonomou, G., Perens, E.A., Lu, Y., and Shaham, S. (2012). Some, but not all, retromer components promote morphogenesis of C. elegans sensory compartments. Dev. Biol. 362, 42-49. Abstract Article

Oikonomou, G., Perens, E.A., Lu, Y., Watanabe, S., Jorgensen, E.M., and Shaham, S. (2011). Opposing activities of LIT-1/NLK and DAF-6/patched-related direct sensory compartment morphogenesis in C. elegans. PLoS Biol. 9, e1001121. Abstract Article

Oiso, N., Riddle, S.R., Serikawa, T., Kuramoto, T., and Spritz, R.A. (2004). The rat Ruby (R) locus is Rab38: identical mutations in Fawn-hooded and Tester-Moriyama rats derived from an ancestral Long Evans rat sub-strain. Mamm. Genome 15, 307-314. Abstract

Olson, S.K., Greenan, G., Desai, A., Muller-Reichert, T., and Oegema, K. (2012). Hierarchical assembly of the eggshell and permeability barrier in C. elegans. J. Cell Biol. 198, 731-748. Abstract Article

Pan, C.L., Baum, P.D., Gu, M., Jorgensen, E.M., Clark, S.G., and Garriga, G. (2008). C. elegans AP-2 and retromer control Wnt signaling by regulating MIG-14/Wntless. Dev. Cell 14, 132-139. Abstract Article

Pant, S., Sharma, M., Patel, K., Caplan, S., Carr, C.M., and Grant, B.D. (2009). AMPH-1/Amphiphysin/Bin1 functions with RME-1/Ehd1 in endocytic recycling. Nat. Cell Biol. 11, 1399-1410. Abstract Article

Patton, A., Knuth, S., Schaheen, B., Dang, H., Greenwald, I., and Fares, H. (2005). Endocytosis function of a ligand-gated ion channel homolog in Caenorhabditis elegans. Curr. Biol. 15, 1045-1050. Abstract Article

Penheiter, S.G., Singh, R.D., Repellin, C.E., Wilkes, M.C., Edens, M., Howe, P.H., Pagano, R.E., and Leof, E.B. (2010). Type II transforming growth factor- $\beta$ receptor recycling is dependent upon the clathrin adaptor protein Dab2. Mol. Biol. Cell 21, 4009-4019. Abstract Article

Pereira-Leal, J.B., and Seabra, M.C. (2001). Evolution of the Rab family of small GTP-binding proteins. J. Mol. Biol. 313, 889-901. Abstract Article

Peters, C., Bayer, M.J., Buhler, S., Andersen, J.S., Mann, M., and Mayer, A. (2001). Trans-complex formation by proteolipid channels in the terminal phase of membrane fusion. Nature 409, 581-588. Abstract Article

Platt, F.M., Boland, B., and van der Spoel, A.C. (2012). The cell biology of disease: lysosomal storage disorders: the cellular impact of lysosomal dysfunction. J. Cell Biol. 199, 723-734. Abstract Article

Pocha, S.M., Wassmer, T., Niehage, C., Hoflack, B., and Knust, E. (2011). Retromer controls epithelial cell polarity by trafficking the apical determinant Crumbs. Curr. Biol. 21, 1111-1117. Abstract Article 
Popoff, V., Mardones, G.A., Bai, S.K., Chambon, V., Tenza, D., Burgos, P.V., Shi, A., Benaroch, P., Urbe, S., Lamaze, C., et al. (2009). Analysis of articulation between clathrin and retromer in retrograde sorting on early endosomes. Traffic 10, 1868-1880. Abstract Article

Port, F., Kuster, M., Herr, P., Furger, E., Bänziger, C., Hausmann, G., and Basler, K. (2008). Wingless secretion promotes and requires retromer-dependent cycling of Wntless. Nat. Cell Biol. 10, 178-185. Abstract Article

Poteryaev, D., Datta, S., Ackema, K., Zerial, M., and Spang, A. (2010). Identification of the switch in early-to-late endosome transition. Cell 141, 497-508. Abstract Article

Poteryaev, D., Fares, H., Bowerman, B., and Spang, A. (2007). Caenorhabditis elegans SAND-1 is essential for RAB-7 function in endosomal traffic. EMBO J. 26, 301-312. Abstract

Poteryaev, D., Squirrell, J.M., Campbell, J.M., White, J.G., and Spang, A. (2005). Involvement of the actin cytoskeleton and homotypic membrane fusion in ER dynamics in Caenorhabditis elegans. Mol. Biol. Cell 16, 2139-2153. Abstract Article

Poupon, V., Girard, M., Legendre-Guillemin, V., Thomas, S., Bourbonniere, L., Philie, J., Bright, N.A., and McPherson, P.S. (2008). Clathrin light chains function in mannose phosphate receptor trafficking via regulation of actin assembly. Proc. Natl. Acad. Sci. U. S. A. 105, 168-173. Abstract Article

Prasad, B.C., and Clark, S.G. (2006). Wnt signaling establishes anteroposterior neuronal polarity and requires retromer in C. elegans. Development 133, 1757-1766. Abstract Article

Puthenveedu, M.A., Lauffer, B., Temkin, P., Vistein, R., Carlton, P., Thorn, K., Taunton, J., Weiner, O.D., Parton, R.G., and von Zastrow, M. (2010). Sequence-dependent sorting of recycling proteins by actin-stabilized endosomal microdomains. Cell 143, 761-773. Abstract Article

Rabbitts, B.M., Ciotti, M.K., Miller, N.E., Kramer, M., Lawrenson, A.L., Levitte, S., Kremer, S., Kwan, E., Weis, A.M., and Hermann, G.J. (2008). glo-3, a novel Caenorhabditis elegans gene, is required for lysosome-related organelle biogenesis. Genetics 180, 857-871. Abstract Article

Radhakrishna, H., and Donaldson, J.G. (1997). ADP-ribosylation factor 6 regulates a novel plasma membrane recycling pathway. J. Cell Biol. 139, 49-61. Abstract Article

Raiborg, C., Bache, K.G., Gillooly, D.J., Madshus, I.H., Stang, E., and Stenmark, H. (2002). Hrs sorts ubiquitinated proteins into clathrin-coated microdomains of early endosomes. Nat. Cell Biol. 4, 394-398. Abstract Article

Raiborg, C., Bache, K.G., Mehlum, A., Stang, E., and Stenmark, H. (2001a). Hrs recruits clathrin to early endosomes. EMBO J. 20, 5008-5021. Abstract Article

Raiborg, C., Bremnes, B., Mehlum, A., Gillooly, D.J., D'Arrigo, A., Stang, E., and Stenmark, H. (2001b). FYVE and coiled-coil domains determine the specific localisation of Hrs to early endosomes. J. Cell Sci. 114, 2255-2263. Abstract

Raiborg, C., Wesche, J., Malerod, L., and Stenmark, H. (2006). Flat clathrin coats on endosomes mediate degradative protein sorting by scaffolding Hrs in dynamic microdomains. J. Cell Sci. 119, 2414-2424. Abstract Article

Richie, C.T., Bembenek, J.N., Chestnut, B., Furuta, T., Schumacher, J.M., Wallenfang, M., and Golden, A. (2011). Protein phosphatase 5 is a negative regulator of separase function during cortical granule exocytosis in C. elegans. J. Cell Sci. 124, 2903-2913. Abstract Article

Rink, J., Ghigo, E., Kalaidzidis, Y., and Zerial, M. (2005). Rab conversion as a mechanism of progression from early to late endosomes. Cell 122, 735-749. Abstract Article

Rivera-Molina, F.E., and Novick, P.J. (2009). A Rab GAP cascade defines the boundary between two Rab GTPases on the secretory pathway. Proc. Natl. Acad. Sci. U. S. A. 106, 14408-14413. Abstract Article

Roberts, B., Clucas, C., and Johnstone, I.L. (2003). Loss of SEC-23 in Caenorhabditis elegans causes defects in oogenesis, morphogenesis, and extracellular matrix secretion. Mol. Biol. Cell 14, 4414-4426. Abstract Article 
Roh, H.C., Collier, S., Guthrie, J., Robertson, J.D., and Kornfeld, K. (2012). Lysosome-related organelles in intestinal cells are a zinc storage site in C. elegans. Cell Metab. 15, 88-99. Abstract Article

Rolls, M.M., Hall, D.H., Victor, M., Stelzer, E.H., and Rapoport, T.A. (2002). Targeting of rough endoplasmic reticulum membrane proteins and ribosomes in invertebrate neurons. Mol. Biol. Cell 13, 1778-1791. Abstract Article

Romer, W., Pontani, L.L., Sorre, B., Rentero, C., Berland, L., Chambon, V., Lamaze, C., Bassereau, P., Sykes, C., Gaus, K., and Johannes, L. (2010). Actin dynamics drive membrane reorganization and scission in clathrin-independent endocytosis. Cell 140, 540-553. Abstract Article

Roudier, N., Lefebvre, C., and Legouis, R. (2005). CeVPS-27 is an endosomal protein required for the molting and the endocytic trafficking of the low-density lipoprotein receptor-related protein 1 in Caenorhabditis elegans. Traffic 6,695-705. Abstract

Ruaud, A.F., Nilsson, L., Richard, F., Larsen, M.K., Bessereau, J.L., and Tuck, S. (2009). The C. elegans P4-ATPase TAT-1 regulates lysosome biogenesis and endocytosis. Traffic 10, 88-100. Abstract Article

Ruck, A., Attonito, J., Garces, K.T., Nunez, L., Palmisano, N.J., Rubel, Z., Bai, Z., Nguyen, K.C., Sun, L., Grant, B.D., et al. (2011). The Atg6/Vps30/Beclin 1 ortholog BEC-1 mediates endocytic retrograde transport in addition to autophagy in C. elegans. Autophagy 7, 386-400. Abstract Article

Samara, C., Syntichaki, P., and Tavernarakis, N. (2008). Autophagy is required for necrotic cell death in Caenorhabditis elegans. Cell Death Differ. 15, 105-112. Abstract Article

Sann, S.B., Crane, M.M., Lu, H., and Jin, Y. (2012). Rabx-5 regulates RAB-5 early endosomal compartments and synaptic vesicles in C. elegans. PLoS One 7, e37930. Abstract Article

Sappington, T.W., and Raikhel, A.S. (1998). Molecular characteristics of insect vitellogenins and vitellogenin receptors. Insect Biochem. Mol. Biol. 28, 277-300. Abstract Article

Sasaki, A., Nakae, I., Nagasawa, M., Hashimoto, K., Abe, F., Saito, K., Fukuyama, M., Gengyo-Ando, K., Mitani, S., Katada, T., and Kontani, K. (2013). Arl8/ARL-8 functions in apoptotic cell removal by mediating phagolysosome formation in Caenorhabditis elegans. Mol. Biol. Cell 24, 1584-1592. Abstract Article

Sasidharan, N., Sumakovic, M., Hannemann, M., Hegermann, J., Liewald, J.F., Olendrowitz, C., Koenig, S., Grant, B.D., Rizzoli, S.O., Gottschalk, A., and Eimer, S. (2012). RAB-5 and RAB-10 cooperate to regulate neuropeptide release in Caenorhabditis elegans. Proc. Natl. Acad. Sci. U. S. A. 109, 18944-18949. Abstract Article

Sato, K., Ernstrom, G.G., Watanabe, S., Weimer, R.M., Chen, C.H., Sato, M., Siddiqui, A., Jorgensen, E.M., and Grant, B.D. (2009). Differential requirements for clathrin in receptor-mediated endocytosis and maintenance of synaptic vesicle pools. Proc. Natl. Acad. Sci. U. S. A. 106, 1139-1144. Abstract Article

Sato, K., Sato, M., Audhya, A., Oegema, K., Schweinsberg, P., and Grant, B.D. (2006). Dynamic regulation of caveolin-1 trafficking in the germ line and embryo of Caenorhabditis elegans. Mol. Biol. Cell 17, 3085-3094. Abstract Article

Sato, M., Grant, B.D., Harada, A., and Sato, K. (2008a). Rab11 is required for synchronous secretion of chondroitin proteoglycans after fertilization in Caenorhabditis elegans. J. Cell Sci. 121, 3177-3186. Abstract Article

Sato, M., Konuma, R., Sato, K., Tomura, K., and Sato, K. (2014). Fertilization-induced K63-linked ubiquitylation mediates clearance of maternal membrane proteins. Development 141, 1324-1331. Abstract Article

Sato, M., Saegusa, K., Sato, K., Hara, T., Harada, A, and Sato K. (2011). Caenorhabditis elegans SNAP-29 is required for organellar integrity of the endomembrane system and general exocytosis in intestinal epithelial cells. Mol. Biol. Cell 22, 2579-2587. Abstract Article

Sato, M., and Sato, K. (2011). Degradation of paternal mitochondria by fertilization-triggered autophagy in $C$. elegans embryos. Science 334, 1141-1144. Abstract Article 
Sato, M., and Sato, K. (2012). Maternal inheritance of mitochondrial DNA: degradation of paternal mitochondria by allogeneic organelle autophagy, allophagy. Autophagy 8, 424-425. Abstract Article

Sato, M., and Sato, K. (2013a). Dynamic regulation of autophagy and endocytosis for cell remodeling during early development. Traffic 14, 479-486. Abstract Article

Sato, M., and Sato, K. (2013b). Maternal inheritance of mitochondrial DNA by diverse mechanisms to eliminate paternal mitochondrial DNA. Biochim Biophys Acta 1833,1979-1984. Abstract Article

Sato, M., Sato, K., Fonarev, P., Huang, C.J., Liou, W., and Grant, B.D. (2005). Caenorhabditis elegans RME-6 is a novel regulator of RAB-5 at the clathrin-coated pit. Nat. Cell Biol. 7, 559-569. Abstract Article

Sato, M., Sato, K., Liou, W., Pant, S., Harada, A., and Grant, B.D. (2008b). Regulation of endocytic recycling by $C$. elegans Rab35 and its regulator RME-4, a coated-pit protein. EMBO J. 27, 1183-1196. Abstract Article

Sato, T., Mushiake, S., Kato, Y., Sato, K., Sato, M., Takeda, N., Ozono, K., Miki, K., Kubo, Y., Tsuji, A., et al. (2007). The Rab8 GTPase regulates apical protein localization in intestinal cells. Nature 448, 366-369. Abstract Article

Sawa H., and Korswagen H.C. (2013). Wnt signaling in C. elegans, WormBook, ed. The C. elegans Research Community, WormBook, doi/10.1895/wormbook.1.7.2, http://www.wormbook.org.

Schaheen, B., Dang, H., and Fares, H. (2009). Derlin-dependent accumulation of integral membrane proteins at cell surfaces. J. Cell Sci. 122, 2228-2239. Abstract Article

Schaheen, L., Dang, H., and Fares, H. (2006a). Basis of lethality in C. elegans lacking CUP-5, the Mucolipidosis Type IV orthologue. Dev. Biol. 293, 382-391. Abstract Article

Schaheen, L., Patton, G., and Fares, H. (2006b). Suppression of the cup-5 mucolipidosis type IV-related lysosomal dysfunction by the inactivation of an ABC transporter in C. elegans. Development 133, 3939-3948. Abstract Article

Schroeder, L.K., Kremer, S., Kramer, M.J., Currie, E., Kwan, E., Watts, J.L., Lawrenson, A.L., and Hermann, G. J. (2007). Function of the Caenorhabditis elegans ABC transporter PGP-2 in the biogenesis of a lysosome-related fat storage organelle. Mol. Biol. Cell 18, 995-1008. Abstract Article

Seaman, M.N. (2012). The retromer complex - endosomal protein recycling and beyond. J. Cell Sci. 125, 4693-4702. Abstract Article

Sebastian, T.T., Baldridge, R.D., Xu, P., and Graham, T.R. (2012). Phospholipid flippases: building asymmetric membranes and transport vesicles. Biochim. Biophys. Acta 1821, 1068-1077. Abstract Article

Semerdjieva, S., Shortt, B., Maxwell, E., Singh, S., Fonarev, P., Hansen, J., Schiavo, G., Grant, B.D., and Smythe, E. (2008). Coordinated regulation of AP2 uncoating from clathrin-coated vesicles by rab5 and hRME-6. J. Cell Biol. 183, 499-511. Abstract Article

Shafaq-Zadah, M., Brocard, L., Solari, F., and Michaux, G. (2012). AP-1 is required for the maintenance of apico-basal polarity in the C. elegans intestine. Development 139, 2061-2070. Abstract Article

Sharrock, W.J. (1983). Yolk proteins of Caenorhabditis elegans. Dev. Biol. 96, 182-188. Abstract Article

Sharrock, W.J., Sutherlin, M.E., Leske, K., Cheng, T.K., and Kim, T.Y. (1990). Two distinct yolk lipoprotein complexes from Caenorhabditis elegans. J. Biol. Chem. 265, 14422-14431. Abstract

Shaye, D.D., and Greenwald, I. (2005). LIN-12/Notch trafficking and regulation of DSL ligand activity during vulval induction in Caenorhabditis elegans. Development 132, 5081-5092. Abstract Article

Sheff, D.R., Kroschewski, R., and Mellman, I. (2002). Actin dependence of polarized receptor recycling in Madin-Darby canine kidney cell endosomes. Mol. Biol. Cell 13, 262-275. Abstract Article 
Shen, X., Steyrer, E., Retzek, H., Sanders, E.J., and Schneider, W.J. (1993). Chicken oocyte growth: receptor-mediated yolk deposition. Cell Tissue Res. 272, 459-471. Abstract

Shi, A., Chen, C.C., Banerjee, R., Glodowski, D., Audhya, A., Rongo, C., and Grant, B.D. (2010). EHBP-1 functions with RAB-10 during endocytic recycling in Caenorhabditis elegans. Mol. Biol. Cell 21, 2930-2943. Abstract Article

Shi, A., Liu, O., Koenig, S., Banerjee, R., Chen, C.C., Eimer, S., and Grant, B.D. (2012). RAB-10-GTPase-mediated regulation of endosomal phosphatidylinositol-4,5-bisphosphate. Proc. Natl. Acad. Sci. U. S. A. 109, E2306-2315. Abstract Article

Shi, A., Pant, S., Balklava, Z., Chen, C.C., Figueroa, V., and Grant, B.D. (2007). A novel requirement for C. elegans Alix/ALX-1 in RME-1-mediated membrane transport. Curr. Biol. 17, 1913-1924. Abstract Article

Shi, A., Sun, L., Banerjee, R., Tobin, M., Zhang, Y., and Grant, B.D. (2009). Regulation of endosomal clathrin and retromer-mediated endosome to Golgi retrograde transport by the J-domain protein RME-8. EMBO J. 28, 3290-3302. Abstract Article

Shim, J., Umemura, T., Nothstein, E., and Rongo, C. (2004). The unfolded protein response regulates glutamate receptor export from the endoplasmic reticulum. Mol. Biol. Cell 15, 4818-4828. Abstract Article

Shnyrova, A.V., Bashkirov, P.V., Akimov, S.A., Pucadyil, T.J., Zimmerberg, J., Schmid, S.L., and Frolov, V.A. (2013). Geometric catalysis of membrane fission driven by flexible dynamin rings. Science 339, 1433-1436. Abstract Article

Silhankova, M., Port, F., Harterink, M., Basler, K., and Korswagen, H.C. (2010). Wnt signalling requires MTM-6 and MTM-9 myotubularin lipid-phosphatase function in Wnt-producing cells. EMBO J. 29, 4094-4105. Abstract Article

Simonsen, A., Bremnes, B., Ronning, E., Aasland, R., and Stenmark, H. (1998). Syntaxin-16, a putative Golgi t-SNARE. Eur. J. Cell Biol. 75, 223-231. Abstract Article

Sitaram, A., Dennis, M.K., Chaudhuri, R., De Jesus-Rojas, W., Tenza, D., Setty, S.R., Wood, C.S., Sviderskaya, E.V., Bennett, D.C., Raposo, G., et al. (2012). Differential recognition of a dileucine-based sorting signal by AP-1 and AP-3 reveals a requirement for both BLOC-1 and AP-3 in delivery of OCA2 to melanosomes. Mol. Biol. Cell 23, 3178-3192. Abstract

Steinberg, F., Gallon, M., Winfield, M., Thomas, E.C., Bell, A.J., Heesom, K.J., Tavare, J.M., and Cullen, P.J. (2013). A global analysis of SNX27-retromer assembly and cargo specicity reveals a function in glucose and metal ion transport. Nat. Cell Biol. 15, 712. Abstract Article

Stifani, S., Barber, D.L., Nimpf, J., and Schneider, W.J. (1990). A single chicken oocyte plasma membrane protein mediates uptake of very low density lipoprotein and vitellogenin. Proc. Natl. Acad. Sci. U. S. A. 87, 1955-1959. Abstract

Strochlic, T.I., Schmiedekamp, B.C., Lee, J., Katzmann, D.J., and Burd, C.G. (2008). Opposing activities of the Snx3-retromer complex and ESCRT proteins mediate regulated cargo sorting at a common endosome. Mol. Biol. Cell 19, 4694-4706. Abstract Article

Strochlic, T.I., Setty, T.G., Sitaram, A., and Burd, C.G. (2007). Grd19/Snx3p functions as a cargo-specific adapter for retromer-dependent endocytic recycling. J. Cell Biol. 177, 115-125. Abstract

Sun, L., Liu, O., Desai, J., Karbassi, F., Sylvain, M.A., Shi, A., Zhou, Z., Rocheleau, C.E., and Grant, B.D. (2012). CED-10/Rac1 regulates endocytic recycling through the RAB-5 GAP TBC-2. PLoS Genet. 8, e1002785. Abstract Article

Sun, T., Wang, X., Lu, Q., Ren, H., and Zhang, H. (2011). CUP-5, the C. elegans ortholog of the mammalian lysosomal channel protein MLN1/TRPML1, is required for proteolytic degradation in autolysosomes. Autophagy 7, 1308-1315. Abstract Article 
Temkin, P., Lauffer, B., Jager, S., Cimermancic, P., Krogan, N.J., and von Zastrow, M. (2011). SNX27 mediates retromer tubule entry and endosome-to-plasma membrane trafficking of signalling receptors. Nat. Cell Biol. 13, 715-721. Abstract Article

Therond, P.P. (2012). Release and transportation of Hedgehog molecules. Curr. Opin. Cell Biol. 24, 173-180. Abstract Article

Thomas, J.H., Stern, M.J., and Horvitz, H.R. (1990). Cell interactions coordinate the development of the C. elegans egg-laying system. Cell 62, 1041-1052. Abstract Article

Tian, X., Hansen, D., Schedl, T., and Skeath, J.B. (2004). Epsin potentiates Notch pathway activity in Drosophila and C. elegans. Development 131, 5807-5815. Abstract Article

Toth, M.L., Sigmond, T., Borsos, E., Barna, J., Erdelyi, P., Takacs-Vellai, K., Orosz, L., Kovacs, A.L., Csikos, G., Sass, M., and Vellai, T. (2008). Longevity pathways converge on autophagy genes to regulate life span in Caenorhabditis elegans. Autophagy 4, 330-338. Abstract

Traub, L.M. (2009). Tickets to ride: selecting cargo for clathrin-regulated internalization. Nat. Rev. Mol. Cell Biol. 10, 583-596. Abstract Article

Traub, L.M., and Lukacs, G.L. (2007). Decoding ubiquitin sorting signals for clathrin-dependent endocytosis by CLASPs. J. Cell Sci. 120, 543-553. Abstract Article

Treusch, S., Knuth, S., Slaugenhaupt, S.A., Goldin, E., Grant, B.D., and Fares, H. (2004). Caenorhabditis elegans functional orthologue of human protein h-mucolipin-1 is required for lysosome biogenesis. Proc. Natl. Acad. Sci. U. S. A. $101,4483-4488$. Abstract

Updike, D., and Strome, S. (2010). P granule assembly and function in Caenorhabditis elegans germ cells. J. Androl. 31, 53-60. Abstract Article

van Meer, G. (2011). Dynamic transbilayer lipid asymmetry. Cold Spring Harb. Perspect. Biol. 3, a004671. Abstract Article

van Weering, J.R., Verkade, P., and Cullen, P.J. (2010). SNX-BAR proteins in phosphoinositide-mediated, tubular-based endosomal sorting. Semin. Cell Dev. Biol. 21, 371-380. Abstract Article

Verges, M. (2007). Retromer and sorting nexins in development. Front. Biosci. 12, 3825-3851. Abstract Article

Voos, W., and Stevens, T.H. (1998). Retrieval of resident late-Golgi membrane proteins from the prevacuolar compartment of Saccharomyces cerevisiae is dependent on the function of Grd19p. J. Cell Biol. 140, 577-590. Abstract Article

Walsh, P., Bursac, D., Law, Y.C., Cyr, D., and Lithgow, T. (2004). The J-protein family: modulating protein assembly, disassembly and translocation. EMBO Rep. 5, 567-571. Abstract Article

Wang, E., Brown, P.S., Aroeti, B., Chapin, S.J., Mostov, K.E., and Dunn, K.W. (2000). Apical and basolateral endocytic pathways of MDCK cells meet in acidic common endosomes distinct from a nearly-neutral apical recycling endosome. Traffic 1, 480-493. Abstract Article

Wang, W., Bouhours, M., Gracheva, E.O., Liao, E.H., Xu, K., Sengar, A.S., Xin, X., Roder, J., Boone, C., Richmond, J.E., et al. (2008). ITSN-1 controls vesicle recycling at the neuromuscular junction and functions in parallel with DAB-1. Traffic 9, 742-754. Abstract Article

Wang, W., and Struhl, G. (2004). Drosophila Epsin mediates a select endocytic pathway that DSL ligands must enter to activate Notch. Development 131, 5367-5380. Abstract Article

Wassmer, T., Attar, N., Harterink, M., van Weering, J.R., Traer, C.J., Oakley, J., Goud, B., Stephens, D.J., Verkade, P., Korswagen, H.C., and Cullen, P.J. (2009). The retromer coat complex coordinates endosomal sorting and dynein-mediated transport, with carrier recognition by the trans-Golgi network. Dev. Cell 17, 110-122. Abstract Article 
Wigge, P., and McMahon, H.T. (1998). The amphiphysin family of proteins and their role in endocytosis at the synapse. Trends Neurosci. 21, 339-344. Abstract Article

Williams, R.L., and Urbe, S. (2007). The emerging shape of the ESCRT machinery. Nat. Rev. Mol. Cell Biol. 8, 355-368. Abstract Article

Winchester, B.G. (2001). Lysosomal membrane proteins. Eur. J. Paediatr. Neurol. 5 Suppl A, 11-19. Abstract

Winter, J.F., Hopfner, S., Korn, K., Farnung, B.O., Bradshaw, C.R., Marsico, G., Volkmer, M., Habermann, B., and Zerial, M. (2012). Caenorhabditis elegans screen reveals role of PAR-5 in RAB-11-recycling endosome positioning and apicobasal cell polarity. Nat. Cell Biol. 14, 666-676. Abstract Article

Witte, K., Schuh, A.L., Hegermann, J., Sarkeshik, A., Mayers, J.R., Schwarze, K., Yates, J.R., 3rd, Eimer, S., and Audhya, A. (2011). TFG-1 function in protein secretion and oncogenesis. Nat. Cell Biol. 13, 550-558. Abstract Article

Wollert, T., and Hurley, J.H. (2010). Molecular mechanism of multivesicular body biogenesis by ESCRT complexes. Nature 464, 864-869. Abstract Article

Wollert, T., Wunder, C., Lippincott-Schwartz, J., and Hurley, J.H. (2009). Membrane scission by the ESCRT-III complex. Nature 458, 172-177. Abstract Article

Woodman, P.G. (2000). Biogenesis of the sorting endosome: the role of Rab5. Traffic 1, 695-701. Abstract Article

Xhabija, B., Taylor, G.S., Fujibayashi, A., Sekiguchi, K., and Vacratsis, P.O. (2011). Receptor mediated endocytosis 8 is a novel $\mathrm{PI}(3) \mathrm{P}$ binding protein regulated by myotubularin-related 2. FEBS Lett. 585, 1722-1728. Abstract Article

Xue, Y., Fares, H., Grant, B., Li, Z., Rose, A.M., Clark, S.G., and Skolnik, E.Y. (2003). Genetic analysis of the myotubularin family of phosphatases in Caenorhabditis elegans. J. Biol. Chem. 278, 34380-34386. Abstract Article

Yang, P.-T., Lorenowicz, M.J., Silhankova, M., Coudreuse, D.Y.M., Betist, M.C., and Korswagen, H.C. (2008a). Wnt signaling requires retromer-dependent recycling of MIG-14/Wntless in Wnt-producing cells. Dev. Cell 14, 140-147. Abstract Article

Yang, P.T., Lorenowicz, M.J., Silhankova, M., Coudreuse, D.Y., Betist, M.C., and Korswagen, H.C. (2008b). Wnt signaling requires retromer-dependent recycling of MIG-14/Wntless in Wnt-producing cells. Dev. Cell 14, 140-147. Abstract Article

Yoshimura, S., Gerondopoulos, A., Linford, A., Rigden, D.J., and Barr, F.A. (2010). Family-wide characterization of the DENN domain Rab GDP-GTP exchange factors. J. Cell Biol. 191, 367-381. Abstract Article

Yu, J.W., and Lemmon, M.A. (2001). All phox homology (PX) domains from Saccharomyces cerevisiae specifically recognize phosphatidylinositol 3-phosphate. J. Biol. Chem. 276, 44179-44184. Abstract Article

Zhang, D., Isack, N.R., Glodowski, D.R., Liu, J., Chen, C.C., Xu, X.Z., Grant, B.D., and Rongo, C. (2012a). RAB-6.2 and the retromer regulate glutamate receptor recycling through a retrograde pathway. J. Cell Biol. 196, 85-101. Abstract Article

Zhang, H., Kim, A., Abraham, N., Khan, L.A., Hall, D.H., Fleming, J.T., and Gobel, V. (2012b). Clathrin and AP-1 regulate apical polarity and lumen formation during C. elegans tubulogenesis. Development 139, 2071-2083. Abstract Article

Zhang, Y., Grant, B., and Hirsh, D. (2001). RME-8, a conserved J-domain protein, is required for endocytosis in Caenorhabditis elegans. Mol. Biol. Cell 12, 2011-2021. Abstract Article

Zhang, Y., Yan, L., Zhou, Z., Yang, P., Tian, E., Zhang, K., Zhao, Y., Li, Z., Song, B., Han, J., et al. (2009). SEPA-1 mediates the specific recognition and degradation of $\mathrm{P}$ granule components by autophagy in C. elegans. Cell 136, 308-321. Abstract Article 
Zhang, P., Wu, Y., Belenkaya, T., and Lin, X. (2011). SNX3 controls Wingless/Wnt secretion through regulating retromer-dependent recycling of Wntless. Cell Res. 21, 1677-1690. Abstract Article

Zhou, B., Wu, Y., and Lin, X. (2011a). Retromer regulates apical-basal polarity through recycling Crumbs. Dev. Biol. 360, 87-95. Abstract Article

Zhou, Q., Li, H., and Xue, D. (2011b). Elimination of paternal mitochondria through the lysosomal degradation pathway in C. elegans. Cell Res 21, 1662-1669. Abstract Article

Zugasti, O., Rajan, J., and Kuwabara, P.E. (2005). The function and expansion of the Patched- and Hedgehog-related homologs in C. elegans. Genome Res 15, 1402-1410. Abstract Article

All WormBook content, except where otherwise noted, is licensed under a Creative SOMIERIGHISRESERVED Commons Attribution License. 